IASSNS-HEP-00/81

\title{
Vertex Algebras, Mirror Symmetry, And D-Branes: The Case Of Complex Tori
}

\author{
Anton Kapustin* Dmitri Orlov ${ }^{\dagger}$
}

\begin{abstract}
A vertex algebra is an algebraic counterpart of a two-dimensional conformal field theory. We give a new definition of a vertex algebra which includes chiral algebras as a special case, but allows for fields which are neither meromorphic nor anti-meromorphic. To any complex torus equipped with a flat Kähler metric and a closed 2-form we associate an $N=2$ superconformal vertex algebra ( $N=2 \mathrm{SCVA}$ ) in the sense of our definition. We find a criterion for two different tori to produce isomorphic $N=2$ SCVA's. We show that for algebraic tori isomorphism of $N=2$ SCVA's implies the equivalence of the derived categories of coherent sheaves corresponding to the tori or their noncommutative generalizations (Azumaya algebras over tori). We also find a criterion for two different tori to produce $N=2$ SCVA's related by a mirror morphism. If the 2 -form is of type $(1,1)$, this condition is identical to the one proposed by Golyshev, Lunts, and Orlov, who used an entirely different approach inspired by the Homological Mirror Symmetry Conjecture of Kontsevich. Our results suggest that Kontsevich's conjecture must be modified: coherent sheaves must be replaced with modules over Azumaya algebras, and the Fukaya category must be "twisted" by a closed 2-form. We also describe the implications of our results for BPS D-branes on Calabi-Yau manifolds.
\end{abstract}

\footnotetext{
${ }^{*}$ School of Natural Sciences, Institute for Advanced Study, Olden Lane, Princeton, NJ 08540, E-mail: kapustin@ias.edu

${ }^{\dagger}$ Algebra Section, Steklov Mathematical Institute, Russian Academy of Sciences, 8 Gubkin str., GSP-1, Moscow 117966, Russia, E-mail: orlov@mi.ras.ru
} 


\section{Contents}

\begin{tabular}{lll}
\hline & Introduction & 2
\end{tabular}

1.1 Physicist's mirror symmetry . . . . . . . . . . . . . . . . . . 2

1.2 Homological mirror symmetry . . . . . . . . . . . . . . . . . . . 4

1.3 Vertex algebras and chiral algebras . . . . . . . . . . . . . . . . . 6

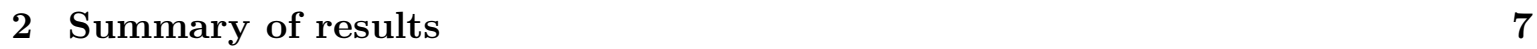

2.1 Physicist's mirror symmetry for complex tori . . . . . . . . . . . . . . 7

2.2 Applications to homological mirror symmetry . . . . . . . . . . . . 8

2.3 Physical applications . . . . . . . . . . . . . . . . . . . . . . . . . . 11

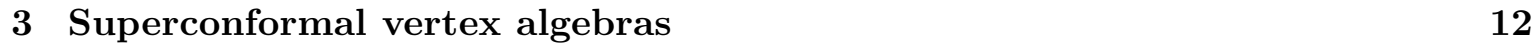

3.1 Quantum fields . . . . . . . . . . . . . . . . . . . . . . . . . . . . . 12

3.2 The definition of a vertex algebra $\ldots \ldots \ldots \ldots \ldots$. . . . . . . . . . . 14

3.3 Conformal vertex algebras . . . . . . . . . . . . . . . . . . . . 18

$3.4 \mathrm{~N}=1$ superconformal vertex algebras $\ldots \ldots \ldots \ldots \ldots \ldots$

$3.5 \mathrm{~N}=2$ superconformal vertex algebras $\ldots \ldots \ldots \ldots \ldots \ldots$

\begin{tabular}{|ll}
\hline $\mathrm{N}=2$ SCVA of a flat complex torus & 22
\end{tabular}

4.1 Vertex algebra structure . . . . . . . . . . . . . . . . . . . . 22

$4.2 \quad N=2$ superconformal structure . . . . . . . . . . . . . . . . . . . 26

\begin{tabular}{|lll}
5 & Morphisms of toroidal superconformal vertex algebras & 27
\end{tabular}

5.1 Isomorphisms of $N=1$ SCVA's . . . . . . . . . . . . . . . . . 27

5.2 Isomorphisms of $N=2$ SCVA' . . . . . . . . . . . . . . . . 33

5.3 Mirror morphisms of $N=2$ SCVA' . . . . . . . . . . . . . . . . 35

\begin{tabular}{|lll}
6 & Homological mirror symmetry with B-fields & $\mathbf{3 6}$
\end{tabular}

6.1 Mirror symmetry and D-branes . . . . . . . . . . . . . . . . 36

6.2 Fukaya category with a B-field $\ldots \ldots \ldots \ldots \ldots$. . . . . . . . . . . 38

\begin{tabular}{|lll|}
\hline A Supersymmetric $\sigma$-model of a flat torus & 43
\end{tabular}

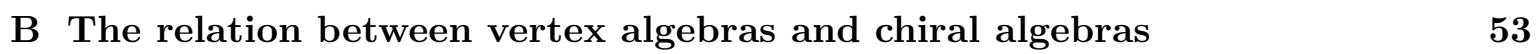

\begin{tabular}{|lll}
\hline C Projectively flat connections and the fundamental group & 63
\end{tabular} 


\section{Introduction}

\subsection{Physicist's mirror symmetry}

A physicist's Calabi Yau is a triple $(X, G, \mathcal{B})$, where $X$ is a compact complex manifold with a trivial canonical bundle, $G$ is a Ricci-flat Kähler metric on $X$, and $\mathcal{B}$ is a class in $H^{2}(X, \mathbb{R} / \mathbb{Z})$ which is in the kernel of the Bockstein homomorphism $H^{2}(X, \mathbb{R} / \mathbb{Z}) \rightarrow$ $H^{3}(X, \mathbb{Z})$. The class $\mathcal{B}$ can be lifted to a class $b \in H^{2}(X, \mathbb{R})$, and a closed 2 -form $B$ representing it is known as the $B$-field.

Physicists believe that there is a procedure which associates to any such triple an $N=2$ superconformal vertex algebra ( $N=2$ SCVA). The precise definition of an $N=2$ SCVA is rather complicated and will be given in Section 3. Roughly speaking, it is a Euclidean quantum field theory on a two-dimensional manifold $\mathbb{R} \times \mathbb{S}^{1}$ whose Hilbert space is acted upon by a unitary representation of the infinite-dimensional Lie super-algebra with even generators $L_{n}, \bar{L}_{n}, J_{n}, \bar{J}_{n}, n \in \mathbb{Z}$, odd generators $Q_{r}^{ \pm}, \bar{Q}_{r}^{ \pm}, r \in \mathbb{Z}+\frac{1}{2}$, and the following nonvanishing Lie brackets:

$$
\begin{array}{llll}
{\left[L_{m}, L_{n}\right]} & =(m-n) L_{m+n}+\frac{d}{4}\left(m^{3}-m\right) \delta_{m,-n}, & \\
{\left[\bar{L}_{m}, \bar{L}_{n}\right]} & =(m-n) \bar{L}_{m+n}+\frac{d}{4}\left(m^{3}-m\right) \delta_{m,-n}, & \\
{\left[L_{m}, J_{n}\right]} & =-n J_{n+m}, & {\left[\bar{L}_{m}, \bar{J}_{n}\right]=-n \bar{J}_{n+m}} \\
{\left[J_{m}, J_{n}\right]} & =d m \delta_{m,-n}, & {\left[\bar{J}_{m}, \bar{J}_{n}\right]=d m \delta_{m,-n}} \\
{\left[L_{m}, Q_{r}^{ \pm}\right]} & =\left(\frac{m}{2}-r\right) Q_{r+m}^{ \pm}, & {\left[\bar{L}_{m}, \bar{Q}_{r}^{ \pm}\right]=\left(\frac{m}{2}-r\right) \bar{Q}_{r+m}^{ \pm}} \\
{\left[J_{m}, Q_{r}^{ \pm}\right]} & = \pm Q_{r+m}^{ \pm}, & \\
\left\{Q_{r}^{+}, Q_{s}^{-}\right\} & =\frac{1}{4} L_{r+s}+\frac{1}{8}(r-s) J_{r+s}+\frac{d}{8}\left(r^{2}-\frac{1}{4}\right) \delta_{r,-s}, & \\
\left\{\bar{Q}_{r}^{+}, \bar{Q}_{s}^{-}\right\} & =\frac{1}{4} \bar{L}_{r+s}+\frac{1}{8}(r-s) \bar{J}_{r+s}+\frac{d}{8}\left(r^{2}-\frac{1}{4}\right) \delta_{r,-s} .
\end{array}
$$

Here $d=\operatorname{dim}_{\mathbb{C}} X$, and $\{\cdot, \cdot\}$ denotes the anti-commutator. This algebra is a direct sum of two copies of the celebrated $N=2$ super-Virasoro algebra with central charge $c=3 d$. If one omits $J_{n}, \bar{J}_{n}$ and all the odd generators from the definition of the $N=2$ SCVA, one gets a structure which we call a conformal vertex algebra (CVA), and which is also known as a conformal field theory on $\mathbb{R} \times \mathbb{S}^{1} \cong \mathbb{C}^{*}$. Thus an $N=2$ SCVA is a conformal field theory on $\mathbb{C}^{*}$ with some additional structure.

Heuristically, the construction of an $N=2$ SCVA from a triplet $(X, G, \mathcal{B})$ proceeds as follows. To any Kähler manifold $(X, G)$ equipped with a closed 2-form $B$ one can associate a two-dimensional classical field theory on $\mathbb{R} \times \mathbb{S}^{1}$, the so-called $N=2$ supersymmetric $\sigma$ model. For reader's convenience, the definition of the $\sigma$-model is given in Appendix A. The space of solutions of the corresponding classical equations of motion is an infinite-dimensional 
symplectic supermanifold with a symplectic action of two copies of the $N=2$ superVirasoro algebra with zero central charge (see [29, 8], and Appendix A). It can be argued that consistent quantization of this classical field theory is possible only for $c_{1}\left(T_{X}\right) \geq 0$, e.g. when $X$ is a Fano manifold or a Calabi-Yau manifold. In the Fano case $\left(c_{1}\left(T_{X}\right)>0\right)$ the quantized $\sigma$-model is an $N=2$ field theory, but not a superconformal one, because only a finite-dimensional subalgebra of the classical $N=2$ super-Virasoro algebra survives quantization. The same happens if $c_{1}\left(T_{X}\right)=0$ but $G$ is not Ricci-flat. If $c_{1}\left(T_{X}\right)=0$ and $G$ is Ricci flat, both $N=2$ super-Virasoro algebras survive quantization (though the central charges become nonzero), and therefore the quantized $\sigma$-model is an $N=2$ superconformal field theory, i.e. an $N=2$ SCVA. One can also argue that this $N=2$ SCVA in fact depends only on the image of $B$ in $H^{2}(X, \mathbb{R} / \mathbb{Z})$, i.e. on $\mathcal{B}$.

The actual quantization of the $\sigma$-model is feasible only for very special $(X, G, \mathcal{B})$. In particular, if $X$ is a complex torus, the corresponding $N=2$ SCVA can be constructed for any flat $G$ and any $\mathcal{B} \in H^{2}(X, \mathbb{R} / \mathbb{Z})$. The quantization of the $\sigma$-model for a flat complex torus is sketched in Appendix A.

Two physicist's Calabi-Yaus are said to be mirror if there exists an isomorphism of the corresponding conformal vertex algebras which acts on the algebra (11) as the so-called mirror involution:

$$
\begin{aligned}
& L_{n} \rightarrow L_{n}, \quad Q_{r}^{ \pm} \rightarrow Q_{r}^{\mp}, \quad J_{n} \rightarrow-J_{n}, \\
& \bar{L}_{n} \rightarrow \bar{L}_{n}, \quad \bar{Q}_{r}^{ \pm} \rightarrow \bar{Q}_{r}^{ \pm}, \quad \bar{J}_{n} \rightarrow \bar{J}_{n} .
\end{aligned}
$$

Such a morphism of $N=2$ SCVA's will be called a mirror morphism.

Mirror symmetry defined in this way acts pointwise on the moduli space of physicist's Calabi-Yaus. If one drops $G$ and $B$ from the definition of a physicist's Calabi-Yau, then mirror symmetry becomes a correspondence between two families of Kähler manifolds with a trivial canonical bundle whose Hodge numbers are related by $h^{p, q}=h^{\prime d-p, q}$. The latter notion of mirror symmetry is much weaker than the physicist's mirror symmetry. Nevertheless, much of the mathematical work on mirror symmetry up to now has focused on this weaker notion, since it proved hard to make sense of the $\sigma$-model.

As mentioned above, the quantum $\sigma$-model is manageable when $X$ is a complex torus, so one could hope to understand mirror symmetry in detail in this particular case. This is what this paper aims to do. Although from the physical point of view mirror symmetry for complex tori appears to be rather trivial, we will see that its study sheds considerable light on the Homological Mirror Symmetry Conjecture (HMSC), a subject to which we now turn. 


\subsection{Homological mirror symmetry}

String theory makes highly nontrivial predictions about the enumerative geometry of a Calabi-Yau $X$ in terms of its mirror $X^{\prime}$. The success of these predictions seems quite mysterious from a purely mathematical standpoint. In an insightful paper [23], M. Kontsevich formulated a conjecture which relates the properties of a Calabi-Yau with those of its mirror and suggested that it captures the essence of mirror symmetry. Subsequently this conjecture was reinterpreted in physical terms [32]. In this subsection we remind the main features of Kontsevich's conjecture.

Let $X$ be a complex algebraic variety (or a complex manifold). Denote by $\mathcal{O}_{X}$ the sheaf of regular functions (or the sheaf of holomorphic functions). Recall that a coherent sheaf is a sheaf of $\mathcal{O}_{X}$-modules that locally can be represented as a cokernel of a morphism of holomorphic vector bundles. Coherent sheaves form an abelian category which will be denoted by $C o h(X)$. To any abelian category we can associate a certain triangulated category called the bounded derived category. We denote by $D^{b}(X)$ the bounded derived category of coherent sheaves on $X$. Roughly speaking, the category $D^{b}(X)$ is a factor-category of the category of bounded complexes of coherent sheaves by the subcategory of acyclic complexes (i.e. complexes with trivial cohomology sheaves).

On the other hand, it has been proposed 12, 23] that to any compact symplectic manifold $Y$ one can associate a certain category whose objects are (roughly speaking) vector bundles on Lagrangian submanifolds equipped with unitary flat connections. The morphisms in this category have been defined when Lagrangian submanifolds intersect transversally. This conjectural category is called the Fukaya category and denoted $\mathcal{F}(Y)$. The category $\mathcal{F}(Y)$ is not an abelian category; rather, it is supposed to be an $A_{\infty}$-category equipped with a shift functor. For an introduction to $A_{\infty}$-categories see [21]. An $A_{\infty}$-category is not a category in the usual sense, because the composition of morphisms is not associative. The set of morphisms between two objects in an $A_{\infty}$-category is a differential graded vector space. To any $A_{\infty}$-category one can associate a true category which has the same objects but the space of morphisms between two objects is the 0 -th cohomology group of the morphisms

in $A_{\infty}$-category. Applying this construction to $\mathcal{F}(Y)$, we obtain a true category $\mathcal{F}_{0}(Y)$ which is also called the Fukaya category. Kontsevich [23] also constructs a certain triangulated category $D \mathcal{F}_{0}(Y)$ out of $\mathcal{F}(Y)$. We will call it the derived Fukaya category. Conjecturally, the category $\mathcal{F}_{0}(Y)$ is a full subcategory of $D \mathcal{F}_{0}(Y)$.

A physicist's Calabi-Yau $(X, G, \mathcal{B})$ is both a complex manifold and a symplectic manifold (the symplectic form being the Kähler form $\omega=G I$ ). Thus we can associate to it a pair of triangulated categories $D^{b}(X)$ and $D \mathcal{F}_{0}(X)$. The Homological Mirror Symmetry Conjecture (HMSC) asserts that if two algebraic Calabi-Yaus $(X, G, \mathcal{B})$ and $\left(X^{\prime}, G^{\prime}, \mathcal{B}^{\prime}\right)$ 
are mirror to each other, then $D^{b}(X)$ is equivalent to $D \mathcal{F}_{0}\left(X^{\prime}\right)$, and vice versa.

The Homological Mirror Symmetry Conjecture can be reinterpreted in physical terms. To every $N=2$ superconformal field theory one can associate the set of BPS D-branes, or more precisely two sets: the set of A-type D-branes and the set of B-type D-branes. This is reviewed in more detail in Section 6. These sets are equipped with a rather intricate algebraic structure: that of an $A_{\infty}$-category. This structure encodes the properties of correlators in a topological open string theory (see [16] and references therein). A mirror morphism between two $N=2$ superconformal field theories identifies the A-type D-branes of the first theory with the B-type D-branes of the second theory, and vice versa. Now suppose that an $N=2$ superconformal field theory originates from a physicist's Calabi-Yau $(X, G, \mathcal{B})$. In this case there is evidence that A-type D-branes are closely related to objects of the Fukaya category, while B-type D-branes are related to coherent sheaves on $X$. To prove the Homological Mirror Symmetry Conjecture it would be sufficient to show that the derived Fukaya category of $(X, G, \mathcal{B})$ (resp. the derived category of $X)$ can be recovered from the $A_{\infty}$-category of A-type D-branes (resp. B-type D-branes). Conversely, proving the HMSC would likely result in an improved understanding of BPS D-branes.

So far the Homological Mirror Symmetry Conjecture (with some important modifications, see Section 6 for details) has been proved only for $\operatorname{dim}_{\mathbb{C}} X=\operatorname{dim} X_{\mathbb{C}}^{\prime}=1$, i.e. for the elliptic curve [31]. Two features make this case particularly manageable. First, the $N=2$ SCVA for the elliptic curve is known, so one knows the precise conditions under which $(X, G, \mathcal{B})$ is mirror to $\left(X^{\prime}, G^{\prime}, \mathcal{B}^{\prime}\right)$. Second, all objects and morphisms in the Fukaya category can be explicitly described.

In this paper we perform a check of the HMSC for the case when both $X$ and $X^{\prime}$ are algebraic tori of arbitrary dimension. We will see that for algebraic tori of dimension higher than one the HMSC as formulated by Kontsevich can not be true in general. The main reason is that both the derived category of coherent sheaves and the derived Fukaya category do not depend on the B-field, while in the physical mirror symmetry it plays an essential role. However, a certain modification of the HMSC which takes into account the B-field passes our check and has a good chance to be correct. This modification is suggested both by our results on the $N=2$ SCVA for complex tori, and by consideration of BPS D-branes. The modified HMSC conjecture is formulated in Section 6. It reduces to the original HMSC when the B-field vanishes for both manifolds related by the mirror morphism.

The implications of our results for BPS D-branes on Calabi-Yau manifolds are briefly described in Section 2 and in more detail in Section 6 . 


\subsection{Vertex algebras and chiral algebras}

Vertex algebras play a key role in physicist's mirror symmetry. A vertex algebra is an algebraic counterpart of a two-dimensional conformal field theory. In the mathematical literature the terms vertex algebra and chiral algebra are used interchangeably. Roughly speaking, a chiral algebra is a vector superspace $V$ together with a map $Y: V \rightarrow \operatorname{End} V\left[\left[z, z^{-1}\right]\right]$ satisfying a number of properties [19]. One says that $Y$ maps states to quantum fields. The definition of a chiral algebra first appeared in the work of Borcherds [6], but its origins go back to the classic paper of Belavin, Polyakov, and Zamolodchikov [5] where an algebraic approach to two-dimensional conformal field theory was proposed.

From a physical viewpoint, chiral algebras are conformal field theories such that all fields are meromorphic (do not depend on $\bar{z}$ ). Only very special conformal field theories have this property. Moreover, a generic conformal field theory does not factorize as a tensor product of two chiral algebras, one depending on $z$ and another on $\bar{z}$, despite some claims to the contrary in the physics literature. For example, the quantization of the $\sigma$-model associated to a flat torus yields a conformal field theory which factorizes in this manner only for very special values of $G$ and $B$.

Thus in order to give a precise meaning to physicist's mirror symmetry, we need to find a sufficiently general definition of a vertex algebra allowing for fields which depend both on $z$ and $\bar{z}$. To avoid confusion, we will refer to these more general objects as vertex algebras, while vertex algebras in the sense of [19] will be called chiral algebras.

Once both $z$ and $\bar{z}$ are allowed, they need not enter only in integer powers, so $Y$ will take values in a space of "fractional power series in $z$ and $\bar{z}$ with coefficients in $\operatorname{End}(V)$ ", rather than in $\operatorname{End}(V)\left[\left[z, \bar{z}, z^{-1}, \bar{z}^{-1}\right]\right]$. The necessity of fractional powers can be seen by inspecting the conformal field theories associated to flat tori. Because of this, the definition of a vertex algebra is not a trivial extension of the definition of a chiral algebra.

We hope that our definition of a vertex algebra will be of some interest to physicists as well as mathematicians. Its advantage over the more standard definitions of conformal field theory is that it is purely algebraic and based on the notion of Operator Product Expansion (OPE). In contrast, other rigorous definitions take Wightman axioms as a starting point. These axioms have an analytic flavor and do not make reference to OPE. In fact, the existence of OPE does not follow from Wightman axioms (except in some very special cases), and has to be postulated separately. Another advantage of our definition is that it does not require an inner product on the state space. Thus it is capable of describing "non-unitary" conformal field theories which find applications in statistical mechanics. 


\section{Summary of results}

\subsection{Physicist's mirror symmetry for complex tori}

Let $T$ be a $2 d$-dimensional real torus $U / \Gamma$, where $U \cong \mathbb{R}^{2 d}$ is a real vector space, and $\Gamma \cong \mathbb{Z}^{2 d}$ is a lattice in $U$. Let $I$ be a (constant) complex structure on $T, G$ be a flat Kähler metric on $T$, and $b \in H^{2}(T, \mathbb{R})$. We will represent $b$ by a constant 2-form $B$ which is uniquely determined by $b$. In this simple case there is a well-known explicit construction of the corresponding $N=2$ SCVA which we denote $\operatorname{Vert}(\Gamma, I, G, B)$. We review this construction in Section 1 . The relation of this construction to the quantized $\sigma$-model is explained in Appendix A.

Our first result describes when two different quadruples $(\Gamma, I, G, B)$ and $\left(\Gamma^{\prime}, I^{\prime}, G^{\prime}, B^{\prime}\right)$ yield isomorphic $N=2$ SCVA's. To state it, we first introduce some notation. Let $\Gamma^{*}=$ $\operatorname{Hom}(\Gamma, \mathbb{Z})$ be the dual lattice in $U^{*}$, and $T^{*}$ be the dual torus $U^{*} / \Gamma^{*}$. There is natural pairing $l: \Gamma \oplus \Gamma^{*} \rightarrow \mathbb{Z}$. There is also a natural $\mathbb{Z}$-valued symmetric bilinear form $q$ on $\Gamma \oplus \Gamma^{*}$ defined by

$$
q\left(\left(w_{1}, m_{1}\right),\left(w_{2}, m_{2}\right)\right)=l\left(w_{1}, m_{2}\right)+l\left(w_{2}, m_{1}\right), \quad w_{1,2} \in \Gamma, m_{1,2} \in \Gamma^{*} .
$$

Given $G, I, B$, we can define two complex structures on $T \times T^{*}$ :

$$
\begin{aligned}
\mathcal{I}(I, B) & =\left(\begin{array}{cc}
I & 0 \\
B I+I^{t} B & -I^{t}
\end{array}\right), \\
\mathcal{J}(G, I, B) & =\left(\begin{array}{cc}
-I G^{-1} B & I G^{-1} \\
G I-B I G^{-1} B & B I G^{-1}
\end{array}\right) .
\end{aligned}
$$

The notation here is as follows. We regard $\mathcal{I}$ and $\mathcal{J}$ as endomorphisms of $U \oplus U^{*}$, and write the corresponding matrices in the basis in which the first $2 d$ vector span $U$, while the remaining vectors span $U^{*}$. In addition, $G$ and $B$ are regarded as elements of $\operatorname{Hom}\left(U, U^{*}\right)$, and $I^{t}$ denotes the endomorphism of $U^{*}$ conjugate to $I$.

It is easy to see that $\mathcal{J}$ depends on $G, I$ only in the combination $\omega=G I$, i.e. it depends only on the symplectic structure on $T$ and the B-field. There is also a third natural complex structure $\tilde{\mathcal{I}}$ on $T \times T^{*}$, which is simply the complex structure that $T \times T^{*}$ gets because it is a Cartesian product of two complex manifolds:

$$
\tilde{\mathcal{I}}=\left(\begin{array}{cc}
I & 0 \\
0 & -I^{t}
\end{array}\right) .
$$

This complex structure will play only a minor role in what follows. Note that $\mathcal{I}$ coincides with $\tilde{\mathcal{I}}$ if and only if $B^{(0,2)}=0$. 
Theorem 2.1 $\operatorname{Vert}(\Gamma, I, G, B)$ is isomorphic to $\operatorname{Vert}\left(\Gamma^{\prime}, I^{\prime}, G^{\prime}, B^{\prime}\right)$ if and only if there exists an isomorphism of lattices $\Gamma \oplus \Gamma^{*}$ and $\Gamma^{\prime} \oplus \Gamma^{*}$ which takes $q$ to $q^{\prime}, \quad \mathcal{I}$ to $\mathcal{I}^{\prime}$, and $\mathcal{J}$ to $\mathcal{J}^{\prime}$.

Our second result describes when $(T, I, G, B)$ is mirror to $\left(T^{\prime}, I^{\prime}, G^{\prime}, B^{\prime}\right)$.

Theorem 2.2 $\operatorname{Vert}(\Gamma, I, G, B)$ is mirror to $\operatorname{Vert}\left(\Gamma^{\prime}, I^{\prime}, G^{\prime}, B^{\prime}\right)$ if and only if there is an isomorphism of lattices $\Gamma \oplus \Gamma^{*}$ and $\Gamma^{\prime} \oplus \Gamma^{\prime *}$ which takes $q$ to $q^{\prime}, \mathcal{I}$ to $\mathcal{J}^{\prime}$, and $\mathcal{J}$ to $\mathcal{I}^{\prime}$.

\subsection{Applications to homological mirror symmetry}

Let us now explain the implications of these results for the HMSC. First, note that if both $B$ and $B^{\prime}$ are of type $(1,1)$, the criterion for mirror symmetry is identical to the one proposed in 14]. In that work, this criterion was taken as a definition of mirror symmetry for algebraic tori. We now see that this definition agrees with the physical notion of mirror symmetry and can be generalized to non-algebraic tori and arbitrary $B$-fields.

Second, Theorem 2.1 allows us to make a check of the HMSC. Suppose the tori $\left(T_{1}, I_{1}, G_{1}, B_{1}\right)$ and $\left(T_{2}, I_{2}, G_{2}, B_{2}\right)$ are both mirror to $\left(T^{\prime}, I^{\prime}, G^{\prime}, B^{\prime}\right)$. Then $\operatorname{Vert}\left(\Gamma_{1}, I_{1}, G_{1}, B_{1}\right)$ is isomorphic to $\operatorname{Vert}\left(\Gamma_{1}, I_{1}, G_{1}, B_{1}\right)$, and by Theorem 2.1 there is an isomorphism of lattices $\Gamma_{1} \oplus \Gamma_{1}^{*}$ and $\Gamma_{2} \oplus \Gamma_{2}^{*}$ which intertwines $q_{1}$ and $q_{2}, \quad \mathcal{I}_{1}$ and $\mathcal{I}_{2}$, and $\mathcal{J}_{1}$ and $\mathcal{J}_{2}$.

On the other hand, if we assume that both $\left(T_{1}, I_{1}\right)$ and $\left(T_{2}, I_{2}\right)$ are algebraic, then HMSC implies that $D^{b}\left(\left(T_{1}, I_{1}\right)\right)$ is equivalent to $D^{b}\left(\left(T_{2}, I_{2}\right)\right)$. The criterion for this equivalence is known [30, 28]: it requires the existence of an isomorphism of $\Gamma_{1} \oplus \Gamma_{1}^{*}$ and $\Gamma_{2} \oplus \Gamma_{2}^{*}$ which intertwines $q_{1}$ and $q_{2}$, and $\tilde{\mathcal{I}}_{1}$ and $\tilde{\mathcal{I}}_{2}$. Clearly, since $\mathcal{I} \neq \tilde{\mathcal{I}}$ in general, this condition is in conflict with the one stated in the end of the previous paragraph. Instead, we only have the following result:

Corollary 2.3 If $\operatorname{Vert}\left(\Gamma_{1}, I_{1}, G_{1}, B_{1}\right)$ is isomorphic to $\operatorname{Vert}\left(\Gamma_{2}, I_{2}, G_{2}, B_{2}\right)$, both $\left(T_{1}, I_{1}\right)$ and $\left(T_{2}, I_{2}\right)$ are algebraic, and both $B_{1}$ and $B_{2}$ are of type $(1,1)$, then $D^{b}\left(\left(T_{1}, I_{1}\right)\right)$ is equivalent to $D^{b}\left(\left(T_{2}, I_{2}\right)\right)$.

In Section 5 we also prove the following result.

Theorem 2.4 Let $\left(T_{1}, I_{1}, G_{1}, B_{1}\right)$ be a complex torus equipped with a flat Kähler metric and a B-field of type $(1,1)$. Let $\left(T_{2}, I_{2}\right)$ be another complex torus. Let $\tilde{\mathcal{I}}_{1}$ and $\tilde{\mathcal{I}}_{2}$ be the product complex structures on $T_{1} \times T_{1}^{*}$ and $T_{2} \times T_{2}^{*}$. Suppose there exists an isomorphism of lattices $g: \Gamma_{1} \oplus \Gamma_{1}^{*} \rightarrow \Gamma_{2} \oplus \Gamma_{2}^{*}$ mapping $q_{1}$ to $q_{2}$ and $\tilde{\mathcal{I}}_{1}$ to $\tilde{\mathcal{I}}_{2}$. Then on $T_{2}$ there 
exists a Kähler metric $G_{2}$ and a B-field $B_{2}$ of type $(1,1)$ such that $\operatorname{Vert}\left(\Gamma_{1}, I_{1}, G_{1}, B_{1}\right)$ is isomorphic to $\operatorname{Vert}\left(\Gamma_{2}, I_{2}, G_{2}, B_{2}\right)$ as an $N=2$ SCVA.

Combining this with Theorem 2.1 and the criterion for the equivalence of $D^{b}\left(\left(T_{1}, I_{1}\right)\right)$ and $D^{b}\left(\left(T_{2}, I_{2}\right)\right)$, we obtain a result converse to Corollary 2.3 .

Corollary 2.5 Let $\left(T_{1}, I_{1}, G_{1}, B_{1}\right)$ be an algebraic torus equipped with a flat Kähler metric and a B-field of type $(1,1)$. Let $\left(T_{2}, I_{2}\right)$ be another algebraic torus. Suppose $D^{b}\left(\left(T_{1}, I_{1}\right)\right)$ is equivalent to $D^{b}\left(\left(T_{2}, I_{2}\right)\right)$. Then on $T_{2}$ there exists a Kähler metric $G_{2}$ and a B-field $B_{2}$ of type $(1,1)$ such that $V \operatorname{ert}\left(\Gamma_{1}, I_{1}, G_{1}, B_{1}\right)$ is isomorphic to $\operatorname{Vert}\left(\Gamma_{2}, I_{2}, G_{2}, B_{2}\right)$ as an $N=2$ SCVA.

If $\operatorname{dim}_{\mathbb{C}} T=1$, then the B-field is automatically of type $(1,1)$. Therefore the HMSC passes the check in this special case. Of course, this is what we expect, since the HMSC is known to be true for the elliptic curve [31]. On the other hand, for $\operatorname{dim}_{\mathbb{C}} T>1$ we seem to have a problem.

Not all is lost however, and a simple modification of the HMSC passes our check. The modification involves replacing $(T, I)$ with a noncommutative algebraic variety, or more precisely, replacing the structure sheaf of $(T, I)$ with an Azumaya algebra over $(T, I)$.

Let us remind the definition and basic facts about Azumaya algebras. Let $\mathcal{A}$ be an $\mathcal{O}_{X}$-algebra which is coherent as a sheaf $\mathcal{O}_{X}$-modules. Denote by $\operatorname{Coh}(\mathcal{A})$ the abelian category of sheaves of (right) $\mathcal{A}$-modules which are coherent as sheaves of $\mathcal{O}_{X}$-modules, and by $D^{b}(\mathcal{A})$ the bounded derived category of $\operatorname{Coh}(\mathcal{A})$.

We will be interested in a simple case of this situation when $\mathcal{A}$ is an Azumaya algebra. Recall that $\mathcal{A}$ is called an Azumaya algebra if it is locally free as a sheaf of $\mathcal{O}_{X}$-modules, and for any point $x \in X$ the restriction $\mathcal{A}(x):=\mathcal{A} \otimes \mathcal{O}_{X} \mathbb{C}(x)$ is isomorphic to a matrix algebra $M_{r}(\mathbb{C})$.

A trivial Azumaya algebra is an algebra of the form $\underline{\mathcal{E} n d}(E)$ where $E$ is a vector bundle. Two Azumaya algebras $\mathcal{A}$ and $\mathcal{A}^{\prime}$ are called similar (or Morita equivalent) if there exist vector bundles $E$ and $E^{\prime}$ such that

$$
\mathcal{A} \otimes_{\mathcal{O}_{X}} \underline{\mathcal{E} n d}(E) \cong \mathcal{A}^{\prime} \otimes_{\mathcal{O}_{X}} \underline{\mathcal{E} n d}\left(E^{\prime}\right)
$$

It is easy to see that in this case the categories $\operatorname{Coh}(\mathcal{A})$ and $\operatorname{Coh}\left(\mathcal{A}^{\prime}\right)$ are equivalent, and therefore the derived categories $D^{b}(\mathcal{A})$ and $D^{b}\left(\mathcal{A}^{\prime}\right)$ are equivalent as well.

Azumaya algebras modulo Morita equivalence generate a group with respect to tensor product. This group is called the Brauer group of the variety and is denoted by $\operatorname{Br}(X)$.

There is a natural map

$$
\operatorname{Br}(X) \longrightarrow H^{2}\left(X, \mathcal{O}_{X}^{*}\right)
$$


This map is an embedding and its image is contained in the torsion subgroup of $H^{2}\left(X, \mathcal{O}_{X}^{*}\right)$. The latter group is denoted by $B r^{\prime}(X)$ and called the cohomological Brauer group of $X$. The well-known Grothendieck conjecture asserts that the natural map $B r(X) \longrightarrow B r^{\prime}(X)$ is an isomorphism for smooth varieties. This conjecture was proved for abelian varieties [17]; we will assume that it is true in general.

Let $X$ be an algebraic variety over $\mathbb{C}$, and let $\mathcal{B} \in H^{2}(X, \mathbb{R} / \mathbb{Z})$. Let $\beta: H^{2}(\mathbb{R} / \mathbb{Z}) \rightarrow$ $H^{2}\left(X, \mathcal{O}_{X}^{*}\right)$ be the homomorphism induced by the canonical map $\mathbb{R} / \mathbb{Z} \longrightarrow \mathcal{O}_{X}^{*}$. We have the following commutative diagram of sheaves:

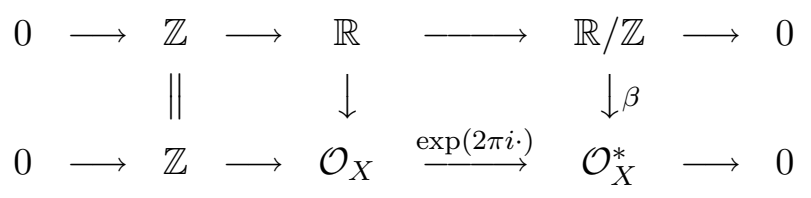

Suppose $\beta(\mathcal{B})$ is a torsion element of $H^{2}\left(X, \mathcal{O}_{X}^{*}\right)$, and consider an Azumaya algebra $\mathcal{A}_{\mathcal{B}}$ which corresponds to this element. The derived category $D^{b}\left(X, \mathcal{A}_{\mathcal{B}}\right)$ does not depend on the choice of $\mathcal{A}_{\mathcal{B}}$ because all these algebras are Morita equivalent. Thus we can denote it simply $D^{b}(X, \mathcal{B})$.

Remark 2.6 It appears that a similar triangulated category can be defined even when $\beta(\mathcal{B})$ is not torsion. Any element $a \in H^{2}\left(X, \mathcal{O}_{X}^{*}\right)$ gives us an $\mathcal{O}_{X}^{*}$ gerbe $\mathcal{X}_{a}$ over $X$. Consider the derived category $D_{Q c o h}^{b}\left(\mathcal{X}_{\beta(\mathcal{B})}\right)$ of quasicoherent sheaves on this gerbe. Now our triangulated category can be defined as a full subcategory of $D_{Q c o h}^{b}\left(\mathcal{X}_{\beta(\mathcal{B})}\right)$ consisting of weight-1 objects with some condition of finiteness, which replaces coherence.

A sufficient condition for the equivalence of $D^{b}\left(X_{1}, \mathcal{B}_{1}\right)$ and $D^{b}\left(X_{2}, \mathcal{B}_{2}\right)$ for the case of algebraic tori is provided by the following theorem [30].

Theorem 2.7 Let $\left(T_{1}, I_{1}\right)$ and $\left(T_{2}, I_{2}\right)$ be two algebraic tori. Let $\mathcal{B}_{1} \in H^{2}\left(T_{1}, \mathbb{R} / \mathbb{Z}\right)$ and $\mathcal{B}_{2} \in H^{2}\left(T_{2}, \mathbb{R} / \mathbb{Z}\right)$, and suppose $\beta$ maps both $\mathcal{B}_{1}$ and $\mathcal{B}_{2}$ to torsion elements. If there exists an isomorphism of lattices $\Gamma_{1} \oplus \Gamma_{1}^{*}$ and $\Gamma_{2} \oplus \Gamma_{2}^{*}$ which maps $q_{1}$ to $q_{2}$, and $\mathcal{I}_{1}$ to $\mathcal{I}_{2}$, then $D^{b}\left(\left(T_{1}, I_{1}\right), \mathcal{B}_{1}\right)$ is equivalent to $D^{b}\left(\left(T_{2}, I_{2}\right), \mathcal{B}_{2}\right)$.

Remark 2.8 It appears plausible that this is also a necessary condition for $D^{b}\left(\left(T_{1}, I_{1}\right), \mathcal{B}_{1}\right)$ to be equivalent to $D^{b}\left(\left(T_{2}, I_{2}\right), \mathcal{B}_{2}\right)$.

Remark 2.9 It appears plausible that the theorem remains true even when $\beta\left(\mathcal{B}_{1}\right)$ and $\beta\left(\mathcal{B}_{2}\right)$ have infinite order, see Remark 2.0.

Combining Theorem 2.7 with our Theorem 2.1, we obtain the following result. 
Corollary 2.10 Suppose $\operatorname{Vert}\left(\Gamma_{1}, I_{1}, G_{1}, B_{1}\right)$ is isomorphic to $\operatorname{Vert}\left(\Gamma_{2}, I_{2}, G_{2}, B_{2}\right)$, both $\left(T_{1}, I_{1}\right)$ and $\left(T_{2}, I_{2}\right)$ are algebraic, and both $\mathcal{B}_{1}$ and $\mathcal{B}_{2}$ are mapped by $\beta$ to torsion elements. Then $D^{b}\left(\left(T_{1}, I_{1}\right), \mathcal{B}_{1}\right)$ is equivalent to $D^{b}\left(\left(T_{2}, I_{2}\right), \mathcal{B}_{2}\right)$.

This corollary suggests that we modify the HMSC by replacing $D^{b}(X)$ with $D^{b}(X, \mathcal{B})$. Once we decided to include the B-field, it seems unnatural to assume that the Fukaya category is independent of it. D-brane considerations suggest a particular way to "twist" the Fukaya category with a B-field (see Section 6). Let us denote this "twisted" category by $\mathcal{F}(Y, \mathcal{B})$. Here $Y$ is a compact symplectic manifold, and $\mathcal{B} \in H^{2}(Y, \mathbb{R} / \mathbb{Z})$ is in the kernel of the Bockstein homomorphism $H^{2}(Y, \mathbb{R} / \mathbb{Z}) \rightarrow H^{3}(Y, \mathbb{Z})$. The modified HMSC asserts that if $(X, G, \mathcal{B})$ is mirror to $\left(X^{\prime}, G^{\prime}, \mathcal{B}^{\prime}\right)$, then $D^{b}(X, \mathcal{B})$ is equivalent to $D \mathcal{F}_{0}\left(X^{\prime}, \mathcal{B}^{\prime}\right)$. Corollary 2.10 shows that this conjecture passes the check which the original HMSC fails.

If both $\mathcal{B}$ and $\mathcal{B}^{\prime}$ vanish, the modified HMSC reduces to the original HMSC. Thus one could ask if it is possible to set the B-field to zero once and for all and work with the original HMSC. This is highly unnatural for the following reason. Suppose we have a mirror pair of physicist's Calabi Yaus which both happen to have zero B-fields. Now let us start varying the complex structure of the first Calabi-Yau. It can be seen in the case of complex tori and can be argued in general that the corresponding deformation of the second Calabi-Yau generally involves both the Kähler form and the B-field. Thus if we have a family of Calabi-Yaus with zero B-field and varying complex structure, the mirror family of Calabi-Yaus will have nonzero B-field for almost all values of the parameter.

For example, in the case of the elliptic curve, the usual Teichmüller parameter $\tau$ takes values in the upper half-plane. The mirror elliptic curve has vanishing $\mathcal{B}$ if and only if $\tau$ can be made purely imaginary by a modular transformation.

In the case of the elliptic curve, the effect of the B-field on the HMSC is relatively minor. It has no effect on the derived category of coherent sheaves because $h^{0,2}=0$. The objects of the Fukaya category are also unmodified in this case (see Section 6), and the only change in the definition of morphisms is to complexify the symplectic form. For higher-dimensional varieties, the modification of the Fukaya category is more serious.

\subsection{Physical applications}

Transformations of the target space metric and the $B$-field which leave the conformal field theory unchanged are known as T-duality transformations. For a real torus $T^{n}=\mathbb{R}^{n} / \Gamma$, $\Gamma \cong \mathbb{Z}^{n}$, such transformations form a group isomorphic to $O(n, n, \mathbb{Z})$ 29, 24]. The main novelty of this work is that we consider complex tori, and study transformations of $G, B$, and the complex structure which leave the $N=2$ superconformal field theory unchanged or induce a mirror morphism. 
Our results have implications for the study of BPS D-branes on Calabi-Yau manifolds, a subject which received much attention recently (see [10] and references therein). They suggest that BPS D-branes of type B are best thought of as objects of the derived category of coherent sheaves when the B-field is zero. When the B-field is nonzero but the corresponding class in $H^{2}\left(X, \mathcal{O}_{X}^{*}\right)$ is a torsion class, the derived category of coherent sheaves should be replaced with the derived category of a certain noncommutative algebraic variety (an Azumaya algebra over $X)$. When the class of the B-field in $H^{2}\left(X, \mathcal{O}_{X}^{*}\right)$ has infinite order, it appears that B-type D-branes should be regarded as objects of the derived category of "coherent" sheaves on a gerbe over $X$.

Note a similarity with the results of [20, 田 where it was shown that in the presence of a Bfield D-brane charges on a smooth manifold $X$ are classified by the K-theory of an Azumaya algebra over $X$, or more generally by the K-theory of a Dixmier-Douady algebra over $X$. The main differences are that Refs. [20, 4] work in a $C^{\infty}$-category, the D-branes are not required to be BPS, and the focus is on D-brane charges rather on D-branes themselves.

In Section 6 we describe the effect of a closed B-field on BPS D-branes of type A (the ones associated to flat unitary bundles on special Lagrangian submanifolds in a Calabi-Yau). This subject was previously studied by Hori et al. [18] for the case of a single D-brane, i.e. when the rank of the bundle is one. Hori et al. find that the restriction of the B-field to the Lagrangian submanifold must vanish. We find that this restriction is too strong: it is sufficient to require the restriction of the B-field to have integer periods. For the higher rank case we argue that in general the unitary bundle on the Lagrangian submanfold is projectively flat rather than flat. Correspondingly, the restrictions on the B-field are even weaker.

\section{Superconformal vertex algebras}

\subsection{Quantum fields}

Let $V$ be a vector superspace over $\mathbb{C}$. The parity of an element $a \in V$ is denoted $p(a)$ and takes values in integers modulo 2 .

Definition 3.1 The space of quantum fields in one formal variable with values in $\operatorname{End}(V)$ is a vector superspace whose elements have the form

$$
\sum_{h \in J} \sum_{n, \bar{n} \in \mathbb{Z}} C_{(h+n, h+\bar{n})} z^{-h-n} \bar{z}^{-h-\bar{n}}
$$

where $J$ is some subset of $[0,1)$ (different for different elements), $C_{(h+n, h+\bar{n})} \in \operatorname{End}(V)$, and the following conditions are satisfied:

a) the set $J$ is countable; 
b) for any element $v \in V$ there is a finite subset $J_{v} \subset J$ such that

$$
C_{(h+n, h+\bar{n})}(v)=0
$$

for all $h \in J \backslash J_{v}$ and all $n, \bar{n} \in \mathbb{Z}$;

c) for any element $v \in V$ there is an integer $N$ such that $C_{h+n, h+\bar{n}}(v)=0$ for all $h \in J$ if $n>N$ or $\bar{n}>N$.

The space of quantum fields in one formal variable with values in $\operatorname{End}(V)$ is denoted $Q F_{1}(V)$.

Given an element $A(z, \bar{z})$ of $Q F_{1}(V)$, we will denote the coefficient of $z^{-h-n} \bar{z}^{-h-\bar{n}}$ in $A(z, \bar{z})$ by $A_{(h+n, h+\bar{n})}$.

The intersection of $Q F_{1}(V)$ with $\operatorname{End}(V)\left[\left[z, z^{-1}\right]\right]$ (resp. $\left.\operatorname{End}(V)\left[\left[\bar{z}, \bar{z}^{-1}\right]\right]\right)$ will be called the space of meromorphic (resp. anti-meromorphic) fields. We will denote by $A(z)$ (resp. $A(\bar{z}))$ meromorphic (resp. anti-meromorphic) fields. The coefficient of $z^{-n}$ in $A(z)$ (resp. the coefficient of $\bar{z}^{-n}$ in $\left.A(\bar{z})\right)$ will be denoted $A_{(n)}$.

Definition 3.2 The space of quantum fields in two formal variables with values in $\operatorname{End}(V)$ is a vector superspace whose elements have the form

$$
\sum_{(h, g) \in J} \sum_{n, \bar{n}, m, \bar{m} \in \mathbb{Z}} C_{(h+n, h+\bar{n}, g+m, g+\bar{m})} z^{-h-n} \bar{z}^{-h-\bar{n}} w^{-g-m} \bar{w}^{-g-\bar{m}}
$$

where $J \in[0,1)^{2}, \quad C_{(h+n, h+\bar{n}, g+m, g+\bar{m})} \in \operatorname{End}(V)$, and the following conditions are satisfied:

$\left.\mathrm{a}^{\prime}\right)$ the set $J$ is countable;

$\left.\mathrm{b}^{\prime}\right)$ for any element $v \in V$ there is a finite subset $J_{v} \subset J$ such that

$$
C_{(h+n, h+\bar{n}, g+m, g+\bar{m})}(v)=0
$$

for all $(h, g) \in J \backslash J_{v}$ and all $n, \bar{n}, m, \bar{m} \in \mathbb{Z}$;

$\left.\mathrm{c}^{\prime}\right)$ for any element $v \in V$ and any $(h, g) \in J$, there is an integer $N$ such that

$$
\begin{aligned}
& C_{(h+n, h+\bar{n}, g+m, g+\bar{m})}(v)=0, \\
& C_{(h+m, h+\bar{m}, g+n, g+\bar{n})}(v)=0,
\end{aligned}
$$

for $n>N$ and any $\bar{n}, m, \bar{m} \in \mathbb{Z}$, and

$$
\begin{aligned}
& C_{(h+n, h+\bar{n}, g+m, g+\bar{m})}(v)=0, \\
& C_{(h+m, h+\bar{m}, g+n, g+\bar{n})}(v)=0,
\end{aligned}
$$

for $\bar{n}>N$ and any $n, m, \bar{m} \in \mathbb{Z}$. 
The space of quantum fields in two formal variables with values in $\operatorname{End}(V)$ is denoted $Q F_{2}(V)$.

Item $\left(\mathrm{c}^{\prime}\right)$ in the definition of $Q F_{2}(V)$ ensures that given an element $C(z, \bar{z}, w, \bar{w})$ of $Q F_{2}(V)$, one can substitute $z=w, \bar{z}=\bar{w}$ and get a well-defined element of $Q F_{1}(V)$. This element will be denoted $C(w, \bar{w}, w, \bar{w})$. Note that in general a product of two fields $A(z, \bar{z}) \in Q F_{1}(V)$ and $B(w, \bar{w}) \in Q F_{1}(V)$ does not belong to $Q F_{2}(V)$, precisely because $\left(\mathrm{c}^{\prime}\right)$ is not satisfied. In this situation one says that the product of $A(z, \bar{z})$ and $B(w, \bar{w})$ has a singularity for $z=w, \bar{z}=\bar{w}$.

If an element $A(z, \bar{z}, w, \bar{w}) \in Q F_{2}(V)$ does not contain nonzero powers of $\bar{z}$ (resp. $z$ ) we will say that this field is meromorphic (resp. anti-meromorphic) in the first variable, and write it as $A(z, w, \bar{w})$ (resp. $A(\bar{z}, w, \bar{w})$ ). Fields in two variables (anti-)meromorphic in the second variable are defined in a similar way.

\subsection{The definition of a vertex algebra}

We set

$$
\begin{aligned}
& i_{z, w} \frac{1}{(z-w)^{h}}=\sum_{j=0}^{\infty}\left(\begin{array}{c}
-h \\
j
\end{array}\right)(-1)^{j} w^{j} z^{-j-h}, \quad i_{\bar{z}, \bar{w}} \frac{1}{(\bar{z}-\bar{w})^{h}}=\sum_{j=0}^{\infty}\left(\begin{array}{c}
-h \\
j
\end{array}\right)(-1)^{j} \bar{w}^{j} \bar{z}^{-j-h}, \\
& i_{w, z} \frac{1}{(z-w)^{h}}=\sum_{j=0}^{\infty}\left(\begin{array}{c}
-h \\
j
\end{array}\right) e^{-i \pi h}(-1)^{j} z^{j} w^{-j-h}, \quad i_{\bar{w}, \bar{z}} \frac{1}{(\bar{z}-\bar{w})^{h}}=\sum_{j=0}^{\infty}\left(\begin{array}{c}
-h \\
j
\end{array}\right) e^{i \pi h}(-1)^{j} \bar{z}^{j} \bar{w}^{-j-h},
\end{aligned}
$$

where

$$
\left(\begin{array}{c}
-h \\
j
\end{array}\right)=\frac{(-h)(-h-1) \cdots(-h-(j-1))}{j !} .
$$

These are formal power series expansions of the functions $(z-w)^{-h}$ and $(\bar{z}-\bar{w})^{-h}$ in the regions $|z|>|w|,|z|<|w|$ and $|\bar{z}|>|\bar{w}|,|\bar{z}|<|\bar{w}|$.

Definition 3.3 A vertex algebra structure on a vector superspace $V$ consists of the following data:

(i) an even vector $|v a c\rangle \in V$.

(ii) a pair $T, \bar{T}$ of commuting even endomorphisms of $V$ annihilating $|v a c\rangle$.

(iii) a parity-preserving linear map

$$
Y: V \rightarrow Q F_{1}(V), \quad Y: a \mapsto Y(a)=a(z, \bar{z})
$$

These data must satisfy the following requirements. 
1. $Y(|v a c\rangle)=i d \in \operatorname{End}(V)$.

2. $[T, a(z, \bar{z})]=\partial a(z, \bar{z}),[\bar{T}, a(z, \bar{z})]=\bar{\partial} a(z, \bar{z})$.

3. $a(z, \bar{z})|v a c\rangle=e^{z T+\bar{z} \bar{T}} a$.

4. For any $a, b \in V$ there are integers $N, M$, real numbers $h_{j} \in[0,1), j=1, \ldots, M$, and quantum fields $C_{j}(z, \bar{z}, w, \bar{w}) \in Q F_{2}(V), j=1, \ldots, M$, such that

$$
\begin{aligned}
a(z, \bar{z}) b(w, \bar{w}) & =\sum_{j=1}^{M} i_{z, w} \frac{1}{(z-w)^{h_{j}+N}} i_{\bar{z}, \bar{w}} \frac{1}{(\bar{z}-\bar{w})^{h_{j}+N}} C_{j}(z, \bar{z}, w, \bar{w}), \\
(-1)^{p(a) p(b)} b(w, \bar{w}) a(z, \bar{z}) & =\sum_{j=1}^{M} i_{w, z} \frac{1}{(z-w)^{h_{j}+N}} i_{\bar{w}, \bar{z}} \frac{1}{(\bar{z}-\bar{w})^{h_{j}+N}} C_{j}(z, \bar{z}, w, \bar{w}) .
\end{aligned}
$$

The map $Y$ is called the state-operator correspondence. The coefficient of $z^{-\alpha} \bar{z}^{-\beta}$ in $Y(a)$ is called the $(\alpha, \beta)$ component of $Y(a)$ and denoted by $a_{(\alpha, \beta)}$.

The last requirement in the definition of a vertex algebra is called the Operator Product Expansion (OPE) axiom. It contains two important ideas. The equality (5) says that the product of two fields in the image of $Y$ has only power-like singularities for $z=w, \bar{z}=\bar{w}$. The difference of (5) and (6) means, roughly speaking, that the fields in the image of $Y$ are mutually local, in the sense that their supercommutator vanishes when $z \neq w$ and $\bar{z} \neq \bar{w}$. This is particularly clear when all $h_{i}$ are equal to zero. Then the supercommutator of $a(z, \bar{z})$ and $b(w, \bar{w})$ is proportional to

$$
\begin{aligned}
\frac{1}{((N-1) !)^{2}} \delta^{(N-1)}(z-w) \delta^{(N-1)}(\bar{z}-\bar{w})+ & \frac{1}{(N-1) !} \delta^{(N-1)}(z-w) i_{\bar{z}, \bar{w}} \frac{1}{(\bar{z}-\bar{w})^{N}} \\
& +\frac{1}{(N-1) !} \delta^{(N-1)}(\bar{z}-\bar{w}) i_{z, w} \frac{1}{(z-w)^{N}}
\end{aligned}
$$

where $\delta^{(k)}(z-w)$ is the $k^{\text {th }}$ derivative of the formal delta-function defined as a formal power series

$$
\delta(z-w)=z^{-1} \sum_{n \in \mathbb{Z}}\left(\frac{z}{w}\right)^{n} .
$$

Given any two elements of $Q F_{1}(V)$, we will say that they are mutually local if for their products the OPE formulas (5.6) hold for some $N, M \in \mathbb{Z}, \quad h_{j} \in[0,1), \quad j=1, \ldots, M$, and $C_{j} \in Q F_{2}(V), \quad j=1, \ldots, M$.

Vertex algebras as defined above are a generalization of chiral algebras as defined in [19] in the following sense. First, any chiral algebra is automatically a vertex algebra, with $\bar{T}=0$ and the image of $Y$ consisting of meromorphic fields only. Second, if we consider the subspace in $V$ consisting of vectors which are mapped to meromorphic fields, the restriction 
of $T$ and $Y$ to this subspace specifies on it the structure of a chiral algebra. Similarly, the restriction of $\bar{T}$ and $Y$ to the anti-meromorphic sector yields another chiral algebra. Moreover, all meromorphic fields supercommute with all anti-meromorphic fields. Thus any vertex algebra contains a pair of commuting chiral subalgebras. All these facts are proved in Appendix B.

The OPE formulas simplify when one of the fields is meromorphic or anti-meromorphic. For example, the OPE of a meromorphic field $a(z), a \in V$, with a general field $b(w, \bar{w}), b \in$ $V$, has the following form (see Appendix B for proof):

$$
\begin{aligned}
a(z) b(w, \bar{w}) & =\sum_{j=1}^{N} i_{z, w} \frac{1}{(z-w)^{j}} D_{j}(w, \bar{w})+: a(z) b(w, \bar{w}): \\
(-1)^{p(a) p(b)} b(w, \bar{w}) a(z) & =\sum_{j=1}^{N} i_{w, z} \frac{1}{(z-w)^{j}} D_{j}(w, \bar{w})+: a(z) b(w, \bar{w}): .
\end{aligned}
$$

Here $N$ is some integer, $D_{j}(w, \bar{w}) \in Q F_{1}(V)$, and $: a(z) b(w, \bar{w}):$ is an element of $Q F_{2}(V)$ defined as follows:

$$
: a(z) b(w, \bar{w}):=a(z)_{+} b(w, \bar{w})+(-1)^{p(a) p(b)} b(w, \bar{w}) a(z)_{-},
$$

where we set

$$
a(z)_{+}=\sum_{n \leq 0} a_{(n)} z^{-n}, \quad a(z)_{-}=\sum_{n>0} a_{(n)} z^{-n}
$$

The field : $a(z) b(w, \bar{w})$ : is called the normal ordered product of $a(z)$ and $b(w, \bar{w})$. Since it belongs to $Q F_{2}(V)$, one can set $z=w$ and get a well-defined field in one variable $: a(w) b(w, \bar{w}):$. The difference between the right-hand side of (8) and $: a(z) b(w, \bar{w}):$ is called the singular part of the OPE.

Similarly, one can define the normal ordered product of an anti-meromorphic field with a general field. The normal ordered product of two general fields is not defined.

Let us consider now the OPE of two meromorphic fields $a(z)$ and $b(z)$. We already mentioned that meromorphic fields form a chiral algebra, thus the OPE (8) simplifies even further:

$$
\begin{aligned}
a(z) b(w) & =\sum_{j=1}^{N} i_{z, w} \frac{1}{(z-w)^{j}} D_{j}(w)+: a(z) b(w): \\
(-1)^{p(a) p(b)} b(w) a(z) & =\sum_{j=1}^{N} i_{w, z} \frac{1}{(z-w)^{j}} D_{j}(w)+: a(z) b(w): .
\end{aligned}
$$


Here $D_{j}(w), j=1, \ldots, N$, are meromorphic elements of $Q F_{1}(V)$. Exchanging $a(z)$ and $b(w)$ we get

$$
\begin{aligned}
b(w) a(z) & =\sum_{j=1}^{N} i_{w, z} \frac{1}{(w-z)^{j}} C_{j}(z)+: b(w) a(z): \\
(-1)^{p(a) p(b)} a(z) b(w) & =\sum_{j=1}^{N} i_{z, w} \frac{1}{(w-z)^{j}} C_{j}(z)+: b(w) a(z):
\end{aligned}
$$

where $C_{j}(z), j=1, \ldots, N$, are meromorphic elements of $Q F_{1}(V)$.

In general, the normal ordered product is not supercommutative, i.e.

$$
: a(z) b(w): \neq(-1)^{p(a) p(b)}: b(w) a(z):
$$

Neither is it associative, in the sense that in general

$$
: a(z): b(z) c(z):: \neq:: a(z) b(z): c(z): .
$$

We will define the normal ordered product of more than two (anti-)meromorphic fields inductively from right to left:

$$
: a_{1}(z) a_{2}(z) \ldots a_{n}(z):=: a_{1}(z): a_{2}(z) \ldots a_{n}(z)::
$$

An important special case where the normal ordered product of meromorphic fields is supercommutative is when the fields $D_{j}(w)$ do not depend on $w$, i.e. are constant endomorphisms of $V$. This follows directly from the above OPE formulas. One can also show that if pairwise OPE's of meromorphic fields $a(z), b(z)$, and $c(z)$ have this property, then their normal ordered product is associative [9]. For example, the normal ordered product of free fermion and free boson fields is supercommutative and associative [19, 9].

Another important special case is the OPE of a meromorphic field and an antimeromorphic field. In this case one can also define two normal ordered products, : $a(z) b(\bar{w})$ : and $: b(\bar{w}) a(z):$. But it follows easily from the equations (8) and analogous equations for the OPE of an anti-meromorphic field and a general field, that in this case the singular part of the OPE vanishes, the normal ordered product coincides with the ordinary product, and that consequently all meromorphic fields supercommute with all anti-meromorphic fields. Thus

$$
: a(z) b(\bar{w}):=(-1)^{p(a) p(b)}: b(\bar{w}) a(z): .
$$

This is discussed in more detail in Appendix B.

The singular part of the OPE of two meromorphic fields $a(z)$ and $b(z)$ completely determines and is determined by the supercommutators of $a_{(n)}$ and $b_{(m)}$ for all $n, m \in \mathbb{Z}$. 
Explicit formulas which enable one to pass from the OPE to the supercommutators and back can found in [19].

When writing the OPE of two meromorphic fields we will use a shortened notation in which only the singular part of the OPE is shown. To indicate this, the equality sign is replaced by $\sim$. In addition, we will only write the first of the OPE's in (8), and correspondingly will omit the symbol $i_{z, w}$, as is common in the physics literature. Similar notation is used for the OPE of two anti-meromorphic fields. Thus instead of

$$
a(z) b(w)=\sum_{j=1}^{N} i_{z, w} \frac{1}{(z-w)^{j}} D_{j}(w)+: a(z) b(w):
$$

we will write

$$
a(z) b(w) \sim \sum_{j=1}^{N} \frac{D_{j}(w)}{(z-w)^{j}} .
$$

We conclude this subsection by defining morphisms of vertex algebras. A morphism from a vertex algebra $(V,|v a c\rangle, T, \bar{T}, Y)$ to a vertex algebra $\left(V^{\prime},|v a c\rangle^{\prime}, T^{\prime}, \bar{T}^{\prime}, Y^{\prime}\right)$ is a morphism of superspaces $f: V \rightarrow V^{\prime}$ such that

$$
f(|v a c\rangle)=|v a c\rangle^{\prime}, \quad f T=T^{\prime} f, \quad f \bar{T}=\bar{T}^{\prime} f
$$

and

$$
Y^{\prime}(f(a)) f(b)=f(Y(a) b) \quad \forall a, b \in V .
$$

\subsection{Conformal vertex algebras}

Definition 3.4 Let $\mathcal{V}=(V,|v a c\rangle, T, \bar{T}, Y)$ be a vertex algebra. Conformal structure on $\mathcal{V}$ is a pair of even vectors $L, \bar{L} \in V$ such that

$$
L(z, \bar{z})=L(z)=\sum_{n \in \mathbb{Z}} L_{n} z^{-n-2}, \quad \bar{L}(z, \bar{z})=\bar{L}(\bar{z})=\sum_{n \in \mathbb{Z}} \bar{L}_{n} \bar{z}^{-n-2} .
$$

(ii) $\quad L_{-1}=T, \bar{L}_{-1}=\bar{T}$.

(iii) $L(z) L(w) \sim \frac{c / 2}{(z-w)^{4}}+\frac{2 L(w)}{(z-w)^{2}}+\frac{\partial L(w)}{z-w}$,

$$
\bar{L}(\bar{z}) \bar{L}(\bar{w}) \sim \frac{\bar{c} / 2}{(\bar{z}-\bar{w})^{4}}+\frac{2 \bar{L}(\bar{w})}{(\bar{z}-\bar{w})^{2}}+\frac{\bar{\partial} \bar{L}(w)}{\bar{z}-\bar{w}} .
$$

(iv) for any $a \in V$

$$
\left[L_{0}, a(z, \bar{z})\right]=z \partial a(z, \bar{z})+\left(L_{0} a\right)(z, \bar{z}), \quad\left[\bar{L}_{0}, a(z, \bar{z})\right]=\bar{z} \bar{\partial} a(z, \bar{z})+\left(\bar{L}_{0} a\right)(z, \bar{z}) .
$$

Here $c, \bar{c} \in \mathbb{C} . \quad A$ vertex algebra with a conformal structure is called a conformal vertex algebra (CVA). 
The numbers $c$ and $\bar{c}$ are called the holomorphic and anti-holomorphic central charges of the CVA. The reason for this name is the following. The OPE's (10) are equivalent to the following commutation relations for all $n, m \in \mathbb{Z}[19]$ :

$$
\begin{aligned}
& {\left[L_{m}, L_{n}\right]=(m-n) L_{m+n}+c \frac{m^{3}-m}{12} \delta_{m,-n},} \\
& {\left[\bar{L}_{m}, \bar{L}_{n}\right]=(m-n) \bar{L}_{m+n}+\bar{c} \frac{m^{3}-m}{12} \delta_{m,-n},} \\
& {\left[L_{n}, \bar{L}_{m}\right]=0 .}
\end{aligned}
$$

Hence the components of $L(z)$ and $\bar{L}(z)$ form two commuting Virasoro algebras. The Virasoro algebra is the unique central extension of the Witt algebra (the algebra of the infinitesimal diffeomorphisms of a circle). In the present case the central charges of the two Virasoro algebras are $c$ and $\bar{c}$.

Note that axiom 3 in the definition of a vertex algebra implies that both $L_{n}$ and $\bar{L}_{n}$ annihilate $|v a c\rangle$ for all $n \geq-1$.

A morphism $f$ from a CVA $(V,|v a c\rangle, Y, L, \bar{L})$ to a CVA $\left(V^{\prime},|v a c\rangle^{\prime}, Y^{\prime}, L^{\prime}, \bar{L}^{\prime}\right)$ is a morphism of the underlying vertex algebras which satisfies

$$
f(L)=L^{\prime}, \quad f(\bar{L})=\bar{L}^{\prime} .
$$

A conformal vertex algebra is almost the same as a conformal field theory. Namely, a physically acceptable conformal field theory is a conformal vertex algebra whose state space $V$ is equipped with a positive-definite Hermitian inner product, and the following additional constraints are satisfied:

(v) The space $V$ splits as a direct sum of the form

$$
\oplus_{j \in J} \mathcal{W}_{j} \otimes \overline{\mathcal{W}}_{j}
$$

where $J$ is a countable set, and $\mathcal{W}_{j}$ and $\overline{\mathcal{W}}_{j}$ are unitary highest-weight modules over the meromorphic and anti-meromorphic Virasoro algebras, respectively.

(vi) The vacuum vector is the only vector in $V$ annihilated by both $L_{0}$ and $\bar{L}_{0}$.

The conformal vertex algebras we will be working with satisfy these constraints and therefore are honest conformal field theories. However, we prefer not to stress the "real" aspects of conformal field theories in this paper.

Furthermore, in order for a conformal field theory to admit a string-theoretic interpretation, it must be defined on a Riemann surface of arbitrary genus. (The above axioms define a conformal field theory in genus zero.) This does not require new data, but imposes additional, so-called sewing, constraints. We will work in genus zero only, and therefore will neglect the sewing constraints. 


\section{4 $\mathrm{N}=1$ superconformal vertex algebras}

Definition 3.5 Let $\mathcal{V}=(V,|v a c\rangle, Y, L, \bar{L})$ be a conformal vertex algebra with central charges $c, \bar{c} . \quad N=1$ superconformal structure on $\mathcal{V}$ is a pair of odd vectors $Q, \bar{Q} \in V$ such that

(i) $Q(z, \bar{z})=Q(z)=\sum_{r \in \mathbb{Z}+\frac{1}{2}} \frac{Q_{r}}{z^{r+3 / 2}}, \quad \bar{Q}(z, \bar{z})=\bar{Q}(\bar{z})=\sum_{r \in \mathbb{Z}+\frac{1}{2}} \frac{\bar{Q}_{r}}{\bar{z}^{r+3 / 2}}$.

(ii) The following OPE's hold true:

$$
\begin{aligned}
L(z) Q(w) & \sim \frac{3}{2} \frac{Q(w)}{(z-w)^{2}}+\frac{\partial Q(w)}{(z-w)}, \\
Q(z) Q(w) & \sim \frac{c / 6}{(z-w)^{3}}+\frac{1}{2} \frac{L(w)}{(z-w)},
\end{aligned}
$$

and similar OPE's for the anti-meromorphic fields with $z, w, c, \partial$ replaced with $\bar{z}, \bar{w}, \bar{c}, \bar{\partial}$.

The fields $Q(z)$ and $\bar{Q}(\bar{z})$ are called left-moving and right-moving supercurrents, respectively. A CVA with an $N=1$ superconformal structure is called an $N=1$ superconformal vertex algebra $(N=1 \quad S C V A)$.

$N=1$ superconformal structure is also known as $(1,1)$ superconformal structure. Omitting $\bar{Q}$, one obtains the definition of $(1,0)$ superconformal structure. Morphisms of $N=1$ SCVA's are defined in an obvious way.

The OPE's of $Q(z), \bar{Q}(\bar{z})$ with themselves and $L(z), \bar{L}(\bar{z})$ are equivalent to the following commutation relations:

$$
\begin{array}{rlrl}
{\left[L_{m}, Q_{r}\right]} & =\left(\frac{m}{2}-r\right) Q_{r+m}, & {\left[\bar{L}_{m}, \bar{Q}_{r}\right]=\left(\frac{m}{2}-r\right) \bar{Q}_{r+m},} \\
\left\{Q_{r}, Q_{s}\right\}=\frac{1}{2} L_{r+s}+\frac{c}{12}\left(r^{2}-\frac{1}{4}\right) \delta_{r,-s}, & \left\{\bar{Q}_{r}, \bar{Q}_{s}\right\}=\frac{1}{2} \bar{L}_{r+s}+\frac{\bar{c}}{12}\left(r^{2}-\frac{1}{4}\right) \delta_{r,-s} .
\end{array}
$$

As usual, the barred generators supercommute with the unbarred ones. Thus $L_{n}, \bar{L}_{n}, Q_{r}, \bar{Q}_{r}$ form an infinite-dimensional Lie super-algebra which is a direct sum of two copies of the $N=1$ super-Virasoro algebra with central charges $c$ and $\bar{c}$.

\section{5 $\mathrm{N}=2$ superconformal vertex algebras}

Definition 3.6 Let $\mathcal{V}=(V,|v a c\rangle, Y, L, \bar{L})$ be a conformal vertex algebra with central charges $c, \bar{c} . \quad N=2$ superconformal structure on $\mathcal{V}$ is a pair of even vectors $J, \bar{J} \in V$ and four odd vectors $Q^{+}, Q^{-}, \bar{Q}^{+}, \bar{Q}^{-} \in V$ such that 
(i)

$$
\begin{array}{ll}
J(z, \bar{z})=J(z)=\sum_{n \in \mathbb{Z}} \frac{J_{n}}{z^{n+1}}, & \bar{J}(z, \bar{z})=\bar{J}(\bar{z})=\sum_{n \in \mathbb{Z}} \frac{\bar{J}_{n}}{\bar{z}^{n+1}}, \\
Q^{+}(z, \bar{z})=Q^{+}(z)=\sum_{r \in \mathbb{Z}+\frac{1}{2}} \frac{Q_{r}^{+}}{z^{r+3 / 2}}, & \bar{Q}^{+}(z, \bar{z})=\bar{Q}^{+}(\bar{z})=\sum_{r \in \mathbb{Z}+\frac{1}{2}} \frac{\bar{Q}_{r}^{+}}{\bar{z}^{r+3 / 2}}, \\
Q^{-}(z, \bar{z})=Q^{-}(z)=\sum_{r \in \mathbb{Z}+\frac{1}{2}} \frac{Q_{r}^{-}}{z^{r+3 / 2}}, & \bar{Q}^{-}(z, \bar{z})=\bar{Q}^{-}(\bar{z})=\sum_{r \in \mathbb{Z}+\frac{1}{2}} \frac{\bar{Q}_{r}^{-}}{\bar{z}^{r+3 / 2}}
\end{array}
$$

(ii) the following OPE's hold true:

$$
\begin{aligned}
L(z) Q^{ \pm}(w) & \sim \frac{3}{2} \frac{Q^{ \pm}(w)}{(z-w)^{2}}+\frac{\partial Q^{ \pm}(w)}{(z-w)}, \\
L(z) J(w) & \sim \frac{J(w)}{(z-w)^{2}}+\frac{\partial J(w)}{(z-w)}, \\
J(z) J(w) & \sim \frac{c / 3}{(z-w)^{2}}, \\
J(z) Q^{ \pm}(w) & \sim \pm \frac{Q^{ \pm}(w)}{(z-w)}, \\
Q^{+}(z) Q^{-}(w) & \sim \frac{c / 12}{(z-w)^{3}}+\frac{1}{4} \frac{J(w)}{(z-w)^{2}}+\frac{1}{8} \frac{\partial J(w)+2 L(w)}{(z-w)}, \\
Q^{ \pm}(z) Q^{ \pm}(w) & \sim 0,
\end{aligned}
$$

and similar OPE's for the anti-meromorphic fields with $z, w, c, \partial$ replaced with $\bar{z}, \bar{w}, \bar{c}, \bar{\partial}$.

The fields $J(z)$ and $\bar{J}(\bar{z})$ are called left-moving and right-moving $R$-currents, the fields $Q^{ \pm}(z)$ and $\bar{Q}^{ \pm}(\bar{z})$ are called left-moving and right-moving supercurrents, respectively. $A$ CVA with $N=2$ superconformal structure is called an $N=2$ superconformal vertex algebra $(N=2$ SCVA).

The above OPE's together with the OPE's for $L(z), \bar{L}(z)$ are equivalent to the commutation relations (11) if we set $c=\bar{c}=3 d$.

$N=2$ superconformal structure is also known as a $(2,2)$ superconformal structure. If one omits the anti-meromorphic currents $\bar{J}(\bar{z}), \bar{Q}^{ \pm}(\bar{z})$, one gets the definition of a $(2,0)$ superconformal structure.

Given an $N=2$ SCVA, one can obtain an $N=1$ SCVA by setting $Q=Q^{+}+Q^{-}$, $\bar{Q}=\bar{Q}^{+}+\bar{Q}^{-}$. Thus an $N=2$ SCVA can be regarded as an $N=1$ SCVA with additional structure.

Morphisms of $N=2$ SCVA's are defined in an obvious way. A mirror morphism between two $N=2$ SCVA's is an isomorphism between the underlying $N=1$ SCVA's which induces the following map on $Q^{ \pm}, \bar{Q}^{ \pm}, J, \bar{J}$ :

$$
\begin{aligned}
& f\left(Q^{+}\right)=Q^{-^{\prime}}, f\left(Q^{-}\right)=Q^{+^{\prime}}, f(J)=-J^{\prime}, \\
& f\left(\bar{Q}^{+}\right)=\bar{Q}^{+^{\prime}}, f\left(\bar{Q}^{-}\right)=\bar{Q}^{-^{\prime}}, f(\bar{J})=\bar{J}^{\prime} .
\end{aligned}
$$


This map acts as an outer automorphism on the algebra (1). A composition of two mirror morphisms is an isomorphism of $N=2$ SCVA's.

\section{$4 \mathrm{~N}=2$ SCVA of a flat complex torus}

The purpose of this section is to describe an $N=2$ SCVA canonically associated to a complex torus endowed with a flat Kähler metric and a constant 2-form. None of this material is new, and everything can be found, in one form or another, in standard string theory textbooks [24, 29]. We simply translate these standard constructions into the language of vertex algebras.

\subsection{Vertex algebra structure}

Let $U$ be a real vector space of dimension $2 d$. Let $\Gamma \cong \mathbb{Z}^{2 d}$ be a lattice in $U$. Let $\Gamma^{*} \subset U^{*}$ be the dual lattice $\operatorname{Hom}(\Gamma, \mathbb{Z})$. Let $T=U / \Gamma, T^{*}=U^{*} / \Gamma^{*}$. Let $G$ be a metric on $U$, i.e. a positive symmetric bilinear form on $U$. Let $B$ be a real skew-symmetric bilinear form on $U$. Let $l$ be the natural pairing $\Gamma \times \Gamma^{*} \rightarrow \mathbb{Z}$. The natural pairing $U \times U^{*} \rightarrow \mathbb{R}$ will be also denoted $l$. Let $\mathbb{Z}^{*}$ be the set of nonzero integers. Let the vectors $e_{1}, \ldots, e_{2 d} \in U$ be the generators of $\Gamma$. The components of an element $w \in \Gamma$ in this basis will be denoted by $w^{i}, i=1, \ldots, 2 d$. The components of an element $m \in \Gamma^{*}$ in the dual basis will be denoted by $m_{i}, i=1, \ldots, 2 d$. We also denote by $G_{i j}, B_{i j}$ the components of $G, B$ in this basis. It will be apparent that the superconformal vertex algebra which we construct does not depend on the choice of basis in $\Gamma$. In the physics literature $\Gamma$ is sometimes referred to as the winding lattice, while $\Gamma^{*}$ is called the momentum lattice.

Consider a triple $(T, G, B)$. To any such triple we will associate a superconformal vertex algebra $\mathcal{V}$ which may be regarded as a quantization of the supersymmetric $\sigma$-model described in Appendix A.

The state space of the vertex algebra $\mathcal{V}$ is

$$
V=\mathcal{H}_{b} \otimes_{\mathbb{C}} \mathcal{H}_{f} \otimes_{\mathbb{C}} \mathbb{C}\left[\Gamma \oplus \Gamma^{*}\right]
$$

Here $\mathcal{H}_{b}$ and $\mathcal{H}_{f}$ are bosonic and fermionic Fock spaces defined below, while $\mathbb{C}\left[\Gamma \oplus \Gamma^{*}\right]$ is the group algebra of $\Gamma \oplus \Gamma^{*}$ over $\mathbb{C}$.

To define $\mathcal{H}_{b}$, consider an algebra over $\mathbb{C}$ with generators $\alpha_{s}^{i}, \bar{\alpha}_{s}^{i}, i=1, \ldots, 2 d, s \in \mathbb{Z}^{*}$ and relations

$$
\left[\alpha_{s}^{i}, \alpha_{p}^{j}\right]=s\left(G^{-1}\right)^{i j} \delta_{s,-p}, \quad\left[\bar{\alpha}_{s}^{i}, \bar{\alpha}_{p}^{j}\right]=s\left(G^{-1}\right)^{i j} \delta_{s,-p}, \quad\left[\alpha_{s}^{i}, \bar{\alpha}_{p}^{j}\right]=0
$$


If $s$ is a positive integer, $\alpha_{-s}^{i}$ and $\bar{\alpha}_{-s}^{i}$ are called left and right bosonic creators, respectively, otherwise they are called left and right bosonic annihilators. Either creators or annihilators are referred to as oscillators.

The space $\mathcal{H}_{b}$ is defined as the space of polynomials of even variables $a_{-s}^{i}, \bar{a}_{-s}^{i}, i=$ $1, \ldots, 2 d, s=1,2, \ldots$, The bosonic oscillator algebra (11) acts on the space $\mathcal{H}_{b}$ via

$$
\begin{array}{lllll}
\alpha_{-s}^{i} & \mapsto a_{-s}^{i}, & \bar{\alpha}_{-s}^{i} & \mapsto \bar{a}_{-s}^{i}, \\
\alpha_{s}^{i} & \mapsto s\left(G^{-1}\right)^{i j} \frac{\partial}{\partial a_{-s}^{j}}, & \bar{\alpha}_{s}^{i} & \mapsto s\left(G^{-1}\right)^{i j} \frac{\partial}{\partial \bar{a}_{-s}^{j}},
\end{array}
$$

for all positive $s$. This is the Fock-Bargmann representation of the bosonic oscillator algebra. The vector $1 \in \mathcal{H}_{b}$ is annihilated by all bosonic annihilators and will be denoted $\left|v a c_{b}\right\rangle$.

The space $\mathcal{H}_{b}$ will be regarded as a $\mathbb{Z}_{2}$-graded vector space with a trivial (purely even) grading. It is clear that $\mathcal{H}_{b}$ can be decomposed as $\mathfrak{H}_{b} \otimes \overline{\mathfrak{H}}_{b}$, where $\mathfrak{H}_{b}$ (resp. $\overline{\mathfrak{H}}_{b}$ ) is the bosonic Fock space defined using only the left (right) bosonic oscillators.

To define $\mathcal{H}_{f}$, consider an algebra over $\mathbb{C}$ with generators $\psi_{s}^{i}, \bar{\psi}_{s}^{i}, i=1, \ldots, 2 d, s \in$ $\mathbb{Z}+\frac{1}{2}$ subject to relations

$$
\left\{\psi_{s}^{i}, \psi_{p}^{j}\right\}=\left(G^{-1}\right)^{i j} \delta_{s,-p}, \quad\left\{\bar{\psi}_{s}^{i}, \bar{\psi}_{p}^{j}\right\}=\left(G^{-1}\right)^{i j} \delta_{s,-p}, \quad\left\{\psi_{s}^{i}, \bar{\psi}_{p}^{j}\right\}=0 .
$$

If $s$ is positive, $\psi_{-s}^{i}$ and $\bar{\psi}_{-s}^{i}$ are called left and right fermionic creators respectively, otherwise they are called left and right fermionic annihilators. Collectively they are referred to as fermionic oscillators.

The space $\mathcal{H}_{f}$ is defined as the space of skew-polynomials of odd variables $\theta_{-s}^{i}, \bar{\theta}_{-s}^{i}, i=$ $1, \ldots, 2 d, s=1 / 2,3 / 2, \ldots$, The fermionic oscillator algebra (12) acts on $\mathcal{H}_{f}$ via

$$
\begin{aligned}
\psi_{-s}^{i} & \mapsto \theta_{-s}^{i}, & \bar{\psi}_{-s}^{i} & \mapsto \bar{\theta}_{-s}^{i}, \\
\psi_{s}^{i} & \mapsto\left(G^{-1}\right)^{i j} \frac{\partial}{\partial \theta_{-s}^{j}}, & \bar{\psi}_{s}^{i} & \mapsto\left(G^{-1}\right)^{i j} \frac{\partial}{\partial \bar{\theta}_{-s}^{j}},
\end{aligned}
$$

for all positive $s \in \mathbb{Z}+\frac{1}{2}$. This is the Fock-Bargmann representation of the fermionic oscillator algebra. The vector $1 \in \mathcal{H}_{f}$ is annihilated by all fermionic annihilators and will be denoted $\left|v a c_{f}\right\rangle$. The fermionic Fock space has a natural $\mathbb{Z}_{2}$ grading such that $\left|v a c_{f}\right\rangle$ is even. It can be decomposed as $\mathfrak{H}_{f} \otimes \overline{\mathfrak{H}}_{f}$, where $\mathfrak{H}_{f}$ (resp. $\quad \overline{\mathfrak{H}}_{f}$ ) is constructed using only the left (right) fermionic oscillators.

For $w \in \Gamma, m \in \Gamma^{*}$ we will denote the vector $w \oplus m \in \mathbb{C}\left[\Gamma \oplus \Gamma^{*}\right]$ by $(w, m)$. We will also use a shorthand $|v a c, w, m\rangle$, for

$$
\left|v a c_{b}\right\rangle \otimes\left|v a c_{f}\right\rangle \otimes(w, m) .
$$

To define $\mathcal{V}$, we have to specify the vacuum vector, $T, \bar{T}$, and the state-operator correspondence $Y$. But first we need to define some auxiliary objects. We define the operators 
$W: V \rightarrow V \otimes \Gamma$ and $M: V \rightarrow V \otimes \Gamma^{*}$ as follows:

$$
W^{i}: b \otimes f \otimes(w, m) \mapsto w^{i}(b \otimes f \otimes(w, m)), \quad M_{i}: b \otimes f \otimes(w, m) \mapsto m_{i}(b \otimes f \otimes(w, m))
$$

We also set

$$
\begin{aligned}
Y^{j}(z) & =\sum_{s=-\infty}^{\infty} \frac{\alpha_{s}^{j}}{s z^{s}}, \\
\bar{Y}^{j}(\bar{z}) & =\sum_{s=-\infty}^{\infty} \frac{\bar{\alpha}_{s}^{j}}{s \bar{z}^{s}}, \\
\partial X^{j}(z) & =\frac{1}{z}\left(G^{-1}\right)^{j k} P_{k}-\partial Y^{j}(z), \\
\bar{\partial} X^{j}(\bar{z}) & =\frac{1}{\bar{z}}\left(G^{-1}\right)^{j k} \bar{P}_{k}-\bar{\partial} \bar{Y}^{j}(\bar{z}), \\
\psi^{j}(z) & =\sum_{r \in \mathbb{Z}+\frac{1}{2}} \frac{\psi_{r}^{j}}{z^{r+1 / 2}}, \\
\bar{\psi}^{j}(\bar{z}) & =\sum_{r \in \mathbb{Z}+\frac{1}{2}} \frac{\bar{\psi}_{r}^{j}}{\bar{z}^{r+1 / 2}},
\end{aligned}
$$

where a prime on a sum over $s$ means that the term with $s=0$ is omitted, and $P_{k}$ and $\bar{P}_{k}$ are defined by

$$
P_{k}=\frac{1}{\sqrt{2}}\left(M_{k}+\left(-B_{k j}-G_{k j}\right) W^{j}\right), \quad \bar{P}_{k}=\frac{1}{\sqrt{2}}\left(M_{k}+\left(-B_{k j}+G_{k j}\right) W^{j}\right) .
$$

Note that we did not define $X^{j}(z, \bar{z})$ themselves, but only their derivatives. The reason is that the would-be field $X^{j}(z, \bar{z})$ contains terms proportional to $\log z$ and $\log \bar{z}$, and therefore does not belong to $Q F_{1}(V)$.

The vacuum vector of $\mathcal{V}$ is defined by

$$
|v a c\rangle=|v a c, 0,0\rangle
$$

The operators $T, \bar{T} \in \operatorname{End}(V)$ are defined by

$$
\begin{aligned}
& T=P_{j} \alpha_{-1}^{j}+\sum_{s=1}^{\infty} G_{j k} \alpha_{-1-s}^{j} \alpha_{s}^{k}+\sum_{r=\frac{1}{2}, \frac{3}{2}, \ldots}\left(r+\frac{1}{2}\right) \psi_{-1-r}^{j} \psi_{r}^{k}, \\
& \bar{T}=\bar{P}_{j} \bar{\alpha}_{-1}^{j}+\sum_{s=1}^{\infty} G_{j k} \bar{\alpha}_{-1-s}^{j} \bar{\alpha}_{s}^{k}+\sum_{r=\frac{1}{2}, \frac{3}{2}, \ldots}\left(r+\frac{1}{2}\right) \bar{\psi}_{-1-r}^{j} \bar{\psi}_{r}^{k} .
\end{aligned}
$$

The state-operator correspondence is defined as follows. The state space $V$ is spanned by vectors of the form

$$
\alpha_{-s_{1}}^{j_{1}} \ldots \alpha_{-s_{n}}^{j_{n}} \bar{\alpha}_{-\bar{s}_{1}}^{\bar{j}_{1}} \ldots \bar{\alpha}_{-\bar{s}_{\bar{n}}}^{\bar{j}_{\bar{n}}} \psi_{-r_{1}}^{i_{1}} \ldots \psi_{-r_{q}}^{i_{q}} \bar{\psi}_{-\bar{r}_{1}}^{\bar{i}_{1}} \ldots \bar{\psi}_{-\bar{r}_{\bar{q}}}^{\bar{i}_{\bar{q}}}|v a c, w, m\rangle,
$$


where $n, \bar{n}, q, \bar{q}$ are nonnegative integers, $s_{1}, \ldots, s_{n}, \bar{s}_{1}, \ldots, \bar{s}_{\bar{n}}$ are positive integers, and $r_{1}, \ldots, r_{q}, \bar{r}_{1}, \ldots, \bar{r}_{\bar{q}}$ are positive half-integers. This vector is mapped by $Y$ to the following quantum field:

$$
\begin{gathered}
\sum_{\left(w^{\prime}, m^{\prime}\right) \in \Gamma \oplus \Gamma^{*}} \epsilon_{w, m^{\prime}} \mathcal{T}(w, m) \operatorname{pr}_{\left(w^{\prime}, m^{\prime}\right)} z^{-2 G^{-1}\left(k, k^{\prime}\right)} \bar{z}^{-2 G^{-1}\left(\bar{k}, \bar{k}^{\prime}\right)} \exp \left(k_{j} Y^{j}(z)_{+}+\bar{k}_{j} \bar{Y}^{j}(\bar{z})_{+}\right) \\
: \prod_{l=1}^{n} \frac{\partial^{s_{l}} X^{j_{l}}(z)}{\left(s_{l}-1\right) !} \prod_{\bar{l}=1}^{\bar{n}} \frac{\partial^{\bar{s}_{\bar{l}}} X^{\bar{j}_{\bar{l}}}(\bar{z})}{\left(\bar{s}_{\bar{l}}-1\right) !} \prod_{t=1}^{q} \frac{\partial^{r_{t}-1 / 2} \psi^{i_{t}}(z)}{\left(r_{t}-\frac{1}{2}\right) !} \prod_{\bar{t}=1}^{\bar{q}} \frac{\partial^{\bar{r}_{\bar{t}}-1 / 2} \bar{\psi}^{\bar{i}_{\bar{t}}}(\bar{z})}{\left(\bar{r}_{\bar{t}}-\frac{1}{2}\right) !}: \\
\exp \left(k_{j} Y^{j}(z)_{-}+\bar{k}_{j} \bar{Y}^{j}(\bar{z})_{-}\right)
\end{gathered}
$$

Here $k, \bar{k}, k^{\prime}, \bar{k}^{\prime}$ are elements of $U^{*}$ defined by

$$
\begin{array}{ll}
k_{j}=\frac{1}{\sqrt{2}}\left(m_{j}+\left(-B_{j k}-G_{j k}\right) w^{k}\right), & \bar{k}_{j}=\frac{1}{\sqrt{2}}\left(m_{j}+\left(-B_{j k}+G_{j k}\right) w^{k}\right), \\
k_{j}^{\prime}=\frac{1}{\sqrt{2}}\left(m_{j}^{\prime}+\left(-B_{j k}-G_{j k}\right) w^{\prime k}\right), & \bar{k}_{j}^{\prime}=\frac{1}{\sqrt{2}}\left(m_{j}^{\prime}+\left(-B_{j k}+G_{j k}\right) w^{\prime k}\right),
\end{array}
$$

the operator $\mathcal{T}(w, m)$ is a translation on the lattice $\Gamma \oplus \Gamma^{*}$ :

$$
\mathcal{T}(w, m):(a, b) \mapsto(a+w, b+m)
$$

and the operators $\operatorname{pr}_{\left(w^{\prime}, m^{\prime}\right)}: V \rightarrow V$ are projections onto the subspace $\mathcal{H}_{b} \otimes \mathcal{H}_{f} \otimes\left(w^{\prime}, m^{\prime}\right)$. Finally, $\epsilon_{w, m^{\prime}}$ is a sign equal to $\exp \left(i \pi l\left(w, m^{\prime}\right)\right)$. We also remind that for any meromorphic quantum field $a(z)$ the fields $a(z)_{+}$and $a(z)_{-}$are defined by (9), and there is a similar definition for the anti-meromorphic fields. Thus $Y^{j}(z)_{ \pm}$and $\bar{Y}^{j}(\bar{z})_{ \pm}$are given by

$$
\begin{aligned}
Y^{j}(z)_{-} & =\sum_{s>0} \frac{\alpha_{s}^{j}}{s z^{s}}, & Y^{j}(z)_{+} & =\sum_{s<0} \frac{\alpha_{s}^{j}}{s z^{s}} \\
\bar{Y}^{j}(\bar{z})_{-} & =\sum_{s>0} \frac{\bar{\alpha}_{s}^{j}}{s \bar{z}^{s}}, & \bar{Y}^{j}(\bar{z})_{+} & =\sum_{s<0} \frac{\bar{\alpha}_{s}^{j}}{s \bar{z}^{s}} .
\end{aligned}
$$

One can easily check that (18) is indeed a well-defined quantum field. Furthermore, the vector (17) is unchanged when the bosonic oscillators are permuted, and is multiplied by the parity of the permutation when the fermionic oscillators are permuted. For the map $Y$ to be well-defined, (18) must have the same property. To see that this is indeed the case, note that the OPE of the fields $\psi^{j}$ and $\partial X^{j}$ is given by

$$
\begin{aligned}
\partial X^{j}(z) \partial X^{k}(w) & \sim \frac{\left(G^{-1}\right)^{j k}}{(z-w)^{2}}, \\
\psi^{j}(z) \psi^{k}(w) & \sim \frac{\left(G^{-1}\right)^{j k}}{(z-w)}, \\
\partial X^{j}(z) \psi^{i}(z) & \sim 0
\end{aligned}
$$


and similarly for the anti-meromorphic fields. It follows that the singular part of the OPE for $\psi^{j}, \bar{\psi}^{j}, \partial X^{j}, \bar{\partial} X^{j}$ and their derivatives is proportional to the identity operator, and therefore their normal ordered product is supercommutative.

To facilitate the understanding of (18), we list a few special cases of the state-operator correspondence.

The state $\alpha_{-s}^{j}|v a c, 0,0\rangle$ is mapped by $Y$ to

$$
\frac{1}{(s-1) !} \partial^{s} X^{j}(z) \text {. }
$$

The state $\bar{\alpha}_{-s}^{j}|v a c, 0,0\rangle$ is mapped to

$$
\frac{1}{(s-1) !} \bar{\partial}^{s} X^{j}(\bar{z}) .
$$

The state $\psi_{-s}^{j}|v a c, 0,0\rangle$ is mapped to

$$
\frac{1}{\left(s-\frac{1}{2}\right) !} \partial^{s-1 / 2} \psi^{j}(z) .
$$

The state $\bar{\psi}_{-s}^{j}|v a c, 0,0\rangle$ is mapped to

$$
\frac{1}{\left(s-\frac{1}{2}\right) !} \bar{\partial}^{s-1 / 2} \bar{\psi}^{j}(\bar{z}) .
$$

The state $|v a c, w, m\rangle$ is mapped to

$$
\begin{array}{r}
\sum_{\left(w^{\prime}, m^{\prime}\right) \in \Gamma \oplus \Gamma^{*}} \epsilon_{w, m^{\prime}} \mathcal{T}(w, m) z^{-2 G^{-1}\left(k, k^{\prime}\right)} \bar{z}^{-2 G^{-1}\left(\bar{k}, \bar{k}^{\prime}\right)} \exp \left(k_{j} Y^{j}(z)_{+}+\bar{k}_{j} \bar{Y}^{j}(\bar{z})_{+}\right) \\
\exp \left(k_{j} Y^{j}(z)_{-}+\bar{k}_{j} \bar{Y}^{j}(\bar{z})_{-}\right) p r_{\left(w^{\prime}, m^{\prime}\right)} .
\end{array}
$$

Checking that $(V,|v a c\rangle, T, \bar{T}, Y)$ satisfies the vertex algebra axioms is a tedious but straightforward exercise which we leave to the reader. Implicitly, the axioms are verified in most textbooks on string theory, for example in [29, 24].

\section{2 $\quad N=2$ superconformal structure}

We first define an $N=1$ superconformal structure on $\mathcal{V}$ by setting

$$
\begin{aligned}
L(z) & =\frac{1}{2}: G(\partial X(z), \partial X(z)):-\frac{1}{2}: G(\psi(z), \partial \psi(z)):, \\
\bar{L}(\bar{z}) & =\frac{1}{2}: G(\bar{\partial} X(\bar{z}), \bar{\partial} X(\bar{z})):-\frac{1}{2}: G(\bar{\psi}(\bar{z}), \bar{\partial} \bar{\psi}(\bar{z})):, \\
Q(z) & =\frac{i}{2 \sqrt{2}}: G(\psi(z), \partial X(z)):, \\
\bar{Q}(\bar{z}) & =\frac{i}{2 \sqrt{2}}: G(\bar{\psi}(\bar{z}), \bar{\partial} X(\bar{z})): .
\end{aligned}
$$


It can be easily checked that all these fields are in the image of $Y$, that $L_{-1}=T, \bar{L}_{-1}=\bar{T}$, and that they satisfy the OPE's specified in the Definition 3.5. The central charges turn out to be $c=\bar{c}=3 d$.

To define an $N=2$ superconformal structure, we need to choose a complex structure $I$ on $U$ with respect to which $G$ is a Kähler metric. Let $\omega=G I$ be the corresponding Kähler form. Then the left-moving supercurrents and the $U(1)$ current are defined as follows:

$$
\begin{aligned}
Q^{ \pm}(z) & =\frac{i}{4 \sqrt{2}}: G(\psi(z), \partial X(z)): \pm \frac{1}{4 \sqrt{2}}: \omega(\psi(z), \partial X(z)):, \\
J(z) & =-\frac{i}{2}: \omega(\psi(z), \psi(z)): .
\end{aligned}
$$

The right-moving currents $\bar{Q}^{ \pm}(\bar{z})$ and $\bar{J}(\bar{z})$ are defined by the same expressions with $\partial X$ replaced by $\bar{\partial} X$ and $\psi$ replaced by $\bar{\psi}$. We omit the check that the OPE's of these currents are as specified in the Definition 3.6. In checking the OPE's the relations (19) are useful.

\section{Morphisms of toroidal superconformal vertex algebras}

\subsection{Isomorphisms of $N=1$ SCVA's}

Let $(T, G, B)$ and $\left(T^{\prime}, G^{\prime}, B^{\prime}\right)$ be a pair of $2 d$-dimensional real tori equipped with flat metrics and constant B-fields. Given $G$ and $B$, we define a flat metric on $T \times T^{*}$ by the formula

$$
\mathcal{G}(G, B)=2\left(\begin{array}{cc}
G-B G^{-1} B & B G^{-1} \\
-G^{-1} B & G^{-1}
\end{array}\right) .
$$

The meaning of this formula is that the value of $\mathcal{G}$ on a pair of vectors $x_{1} \oplus y_{1}$ and $x_{2} \oplus y_{2}$, $x_{i} \in U, y_{i} \in U^{*}, i=1,2$, is

$$
2\left(\begin{array}{ll}
x_{1} & y_{1}
\end{array}\right)\left(\begin{array}{cc}
G-B G^{-1} B & B G^{-1} \\
-G^{-1} B & G^{-1}
\end{array}\right)\left(\begin{array}{l}
x_{2} \\
y_{2}
\end{array}\right) .
$$

$\mathcal{G}(G, B)$ is obviously a symmetric form on $U \oplus U^{*}$, and its positive-definiteness follows from the positive-definiteness of $G$ and the identity

$$
\mathcal{G}=R(G, B)^{t}\left(\begin{array}{ll}
G & 0 \\
0 & G
\end{array}\right) R(G, B),
$$

where

$$
R(G, B)=\left(\begin{array}{cc}
-1-G^{-1} B & G^{-1} \\
1-G^{-1} B & G^{-1}
\end{array}\right)
$$


We will use a shorthand $\mathcal{G}(G, B)=\mathcal{G}$ and $\mathcal{G}\left(G^{\prime}, B^{\prime}\right)=\mathcal{G}^{\prime}$. Recall also that we have canonical $\mathbb{Z}$-valued symmetric bilinear forms on $\Gamma \oplus \Gamma^{*}$ and $\Gamma^{\prime} \oplus \Gamma^{*}$ denoted by $q$ and $q^{\prime}$, respectively (see Section 2).

In this subsection we prove

Theorem 5.1 $N=1$ SCVA's corresponding to $(T, G, B)$ and $\left(T^{\prime}, G^{\prime}, B^{\prime}\right)$ are isomorphic if and only if there exists an isomorphism of lattices $\Gamma \oplus \Gamma^{*}$ and $\Gamma^{\prime} \oplus \Gamma^{\prime *}$ which takes $q$ to $q^{\prime}$, and $\mathcal{G}$ to $\mathcal{G}^{\prime}$.

The "if" part of this theorem is proved in many string theory papers, see for example 25, 34. Below we outline a construction of the isomorphism of $N=1$ SCVA's given an isomorphism of lattices and then prove the "only if" part of the theorem.

Let $g$ be an isomorphism of $\Gamma \oplus \Gamma^{*}$ with $\Gamma^{\prime} \oplus \Gamma^{\prime *}$. We will write it as follows:

$$
g=\left(\begin{array}{ll}
a & b \\
c & d
\end{array}\right)
$$

where $a \in \operatorname{Hom}\left(\Gamma, \Gamma^{\prime}\right), \quad b \in \operatorname{Hom}\left(\Gamma^{*}, \Gamma^{\prime}\right), \quad c \in \operatorname{Hom}\left(\Gamma, \Gamma^{\prime *}\right), \quad d \in \operatorname{Hom}\left(\Gamma^{*}, \Gamma^{* *}\right)$. The "realified" maps from $U, U^{*}$ to $U^{\prime}, U^{\prime *}$ will be denoted by the same letters. Let us also set $H=G+B$. Both $V$ and $V^{\prime}$ are tensor products of the group algebra of the respective lattice and bosonic and fermionic Fock spaces. The vertex algebra isomorphism $f: V \rightarrow V^{\prime}$ respects this tensor product structure. $\mathbb{C}\left[\Gamma \oplus \Gamma^{*}\right]$ is mapped to $\mathbb{C}\left[\Gamma^{\prime} \oplus \Gamma^{\prime *}\right]$ in an obvious way:

$$
f:\left(\begin{array}{l}
w \\
m
\end{array}\right) \mapsto\left(\begin{array}{ll}
a & b \\
c & d
\end{array}\right)\left(\begin{array}{l}
w \\
m
\end{array}\right) .
$$

The mapping of Fock spaces is defined by the substitutions

$$
\begin{array}{ll}
\left(\begin{array}{l}
a_{-s}^{i} \\
\bar{a}_{-s}^{i}
\end{array}\right) \mapsto \mathcal{M}(g, H)_{j}^{i}\left(\begin{array}{l}
a_{-s}^{j} \\
\bar{a}_{-s}^{j}
\end{array}\right), & s=1,2, \ldots, \\
\left(\begin{array}{l}
\theta_{-s}^{i} \\
\bar{\theta}_{-s}^{i}
\end{array}\right) \mapsto \mathcal{M}(g, H)_{j}^{i}\left(\begin{array}{l}
\theta_{-s}^{j} \\
\bar{\theta}_{-s}^{j}
\end{array}\right), & s=\frac{1}{2}, \frac{3}{2}, \ldots,
\end{array}
$$

where

$$
\mathcal{M}(g, H)=\left(\begin{array}{cc}
a-b H^{t} & 0 \\
0 & a+b H
\end{array}\right) .
$$

In particular, $f$ preserves the bosonic and fermionic vacuum vectors.

Let us now indicate why this mapping is an isomorphism of $N=1$ SCVA's. The statement that $g$ takes $q$ to $q^{\prime}$ is equivalent to

$$
a^{t} c+c^{t} a=b^{t} d+d^{t} b=0, \quad a^{t} d+c^{t} b=i d_{\Gamma^{*}},
$$


where $a^{t}$ denotes the conjugate of $a$, etc.

The statement that $g$ takes $\mathcal{G}$ to $\mathcal{G}^{\prime}$ is equivalent to

$$
H^{\prime}=(c+d H)(a+b H)^{-1}
$$

where $H^{\prime}=G^{\prime}+B^{\prime}, H=G+B$. To show this, let us denote the right-hand side of the above equation by $H^{\prime \prime}$, let $G^{\prime \prime}$ and $B^{\prime \prime}$ be the symmetric an anti-symmetric parts of $H^{\prime \prime}$, and let $\mathcal{G}^{\prime \prime}=\mathcal{G}\left(G^{\prime \prime}, B^{\prime \prime}\right)$. In view of (20) we have

$$
\mathcal{G}^{\prime \prime}=R\left(G^{\prime \prime}, B^{\prime \prime}\right)^{t}\left(\begin{array}{cc}
G^{\prime \prime} & 0 \\
0 & G^{\prime \prime}
\end{array}\right) R\left(G^{\prime \prime}, B^{\prime \prime}\right) .
$$

Let us multiply this equation by $g^{t}$ from the left and by $g$ from the right and use the identity

$$
R\left(G^{\prime \prime}, B^{\prime \prime}\right) g=\mathcal{M}(g, H) R(G, B)
$$

which can be easily proved using (21). We get

$$
g^{t} \mathcal{G}^{\prime \prime} g=R(G, B)^{t} \mathcal{M}(g, H)^{t}\left(\begin{array}{cc}
G^{\prime \prime} & 0 \\
0 & G^{\prime \prime}
\end{array}\right) \mathcal{M}(g, H) R(G, B) .
$$

We now use another easily checked identity:

$$
G^{\prime \prime}=\left[(a+b H)^{t}\right]^{-1} G(a+b H)^{-1}=\left[\left(a-b H^{t}\right)^{t}\right]^{-1} G\left(a-b H^{t}\right)^{-1},
$$

and obtain

$$
g^{t} \mathcal{G}^{\prime \prime} g=\mathcal{G}
$$

On the other hand, we know that $g^{t} \mathcal{G}^{\prime} g=\mathcal{G}$. Thus $\mathcal{G}^{\prime \prime}=\mathcal{G}^{\prime}$, and hence $G^{\prime \prime}=G^{\prime}, B^{\prime \prime}=$ $B^{\prime}, H^{\prime \prime}=H^{\prime}$. This proves (22). As a consequence of $G^{\prime \prime}=G^{\prime}$ and (24), we obtain a useful formula relating $G^{\prime}$ and $G$ :

$$
G^{\prime}=\left[(a+b H)^{t}\right]^{-1} G(a+b H)^{-1}=\left[\left(a-b H^{t}\right)^{t}\right]^{-1} G\left(a-b H^{t}\right)^{-1} .
$$

Using these relations, one can easily check that the map $f$ intertwines $Y$ and $Y^{\prime}$, i.e.

$$
Y^{\prime}(f(a), z, \bar{z})=f Y(a, z, \bar{z}) f^{-1}, \quad \forall a \in V .
$$

In particular, we have

$$
\begin{gathered}
f^{-1}\left(\begin{array}{l}
\partial X^{\prime i}(z) \\
\bar{\partial} X^{\prime i}(z)
\end{array}\right) f=\mathcal{M}(g, H)_{j}^{i}\left(\begin{array}{l}
\partial X^{j}(z) \\
\bar{\partial} X^{j}(z)
\end{array}\right), \\
f^{-1}\left(\begin{array}{l}
\psi^{\prime i}(z) \\
\bar{\psi}^{\prime}(z)
\end{array}\right) f=\mathcal{M}(g, H)_{j}^{i}\left(\begin{array}{l}
\psi^{j}(z) \\
\bar{\psi}^{j}(z)
\end{array}\right) .
\end{gathered}
$$


These relations and the definition of $L(z), \bar{L}(\bar{z}), Q(z), \bar{Q}(\bar{z})$ imply that the $N=1$ superconformal structure is also preserved:

$$
\begin{aligned}
L^{\prime}(z) & =f L(z) f^{-1}, & Q^{\prime}(z) & =f Q(z) f^{-1} \\
\bar{L}^{\prime}(\bar{z}) & =f \bar{L}(\bar{z}) f^{-1}, & \bar{Q}^{\prime}(\bar{z}) & =f \bar{Q}(\bar{z}) f^{-1}
\end{aligned}
$$

Hence $f$ is an isomorphism of $N=1$ superconformal vertex algebras.

In the remainder of this subsection we prove the "only if" part of the theorem. Let $(T, G, B)$ and $\left(T^{\prime}, G^{\prime}, B^{\prime}\right)$ be two real tori equipped with a flat metric and a constant B-field. Thus $T=U / \Gamma$ and $T^{\prime}=U^{\prime} / \Gamma^{\prime}$, where $U$ and $U^{\prime}$ are real vector spaces and $\Gamma$ and $\Gamma^{\prime}$ are lattices of maximal rank in the respective spaces. Clearly, for the $N=1$ SCVA's to be isomorphic, the central charges of the corresponding super-Virasoro algebras must agree, hence $\operatorname{dim} U=\operatorname{dim} U^{\prime}$. We pick an isomorphism of $U$ and $U^{\prime}$ and a basis in $U$. Let $\mathcal{V}=(V, Y,|v a c\rangle, L, \bar{L}, Q, \bar{Q})$ and $\mathcal{V}^{\prime}=\left(V^{\prime}, Y^{\prime},\left|v a c^{\prime}\right\rangle, L^{\prime}, \bar{L}^{\prime}, Q^{\prime}, \bar{Q}^{\prime}\right)$ be the corresponding $N=1$ SCVA's. Let $f: V \rightarrow V^{\prime}$ be an isomorphism of $N=1$ SCVA's. This means that the equations (26) and (28) hold true. In particular, $f$ preserves the form of the OPE.

Consider the "Hamiltonians" $L_{0}, \bar{L}_{0} \in \operatorname{End}(V)$. A short computation yields:

$$
L_{0}=\frac{1}{8} \mathcal{G}(Z, Z)-\frac{1}{4} q(Z, Z)+N_{b}+N_{f}, \quad \bar{L}_{0}=\frac{1}{8} \mathcal{G}(Z, Z)+\frac{1}{4} q(Z, Z)+\bar{N}_{b}+\bar{N}_{f}
$$

Here $Z=(W, M)$ is regarded as an element of $\operatorname{End}(\mathcal{H}) \otimes_{\mathbb{R}}\left(U \oplus U^{*}\right)$, and we defined

$$
\begin{array}{ll}
N_{b}=\sum_{s=1}^{\infty} G\left(\alpha_{-s}, \alpha_{s}\right), & N_{f}=\sum_{r=1 / 2,3 / 2, \ldots} r G\left(\psi_{-r}, \psi_{r}\right), \\
\bar{N}_{b}=\sum_{s=1}^{\infty} G\left(\bar{\alpha}_{-s}, \bar{\alpha}_{s}\right), & \bar{N}_{f}=\sum_{r=1 / 2,3 / 2, \ldots} r G\left(\bar{\psi}_{-r}, \bar{\psi}_{r}\right) .
\end{array}
$$

The operators $N_{b}, N_{f}, \bar{N}_{b}, \bar{N}_{f}$ commute with each other. For what follows it is important to know their spectrum in Fock space. One can show that the Fock space decomposes into a tensor sum of the joint eigenspaces of $N_{b}, N_{f}, \bar{N}_{b}, \bar{N}_{f}$, and that all the eigenvalues are nonnegative. Furthermore, the spectrum of $N_{b}, \bar{N}_{b}$ is integer, and the spectrum of $N_{f}, \bar{N}_{f}$ is half-integer. Finally, the only vector in $\mathfrak{H}_{b} \otimes \mathfrak{H}_{f}$ annihilated by all four operators is $\left|v a c_{b}\right\rangle \otimes\left|v a c_{f}\right\rangle$. (All of these facts are standard and can be easily proved using the commutation relations for the oscillators.)

Note also that the spectrum of the operator $\mathcal{G}(Z, Z)$ is nonnegative because $\mathcal{G}$ is a positive-definite form. The only vector in $\mathbb{C}\left[\Gamma \oplus \Gamma^{*}\right]$ annihilated by $\mathcal{G}(Z, Z)$ is $(0,0)$.

Now let us find all the eigenvectors of $L_{0}, \bar{L}_{0}$ with eigenvalues $(1 / 2,0)$. Suppose $a \in V$ is such an eigenvector. Since $L_{0}, \bar{L}_{0}$ commute with $Z=(W, M)$, we may assume that a 
is an eigenvector of $Z$ with an eigenvalue $z=(w, m)$, where $w \in \Gamma, m \in \Gamma^{*}$. In view of the above we have three possibilities:

Case 1. $\quad N_{b} a=\bar{N}_{b} a=N_{f} a=\bar{N}_{f} a=0$,

$$
\frac{1}{2} \mathcal{G}(z, z)-q(z, z)=2, \quad \frac{1}{2} \mathcal{G}(z, z)+q(z, z)=0
$$

Case 2. $\quad N_{b} a=\bar{N}_{b} a=N_{f} a=0$

$$
\left(\bar{N}_{f}-\frac{1}{2}\right) a=0
$$

$$
\mathcal{G}(z, z)=q(z, z)=0
$$

Case 3. $\quad N_{b} a=\bar{N}_{b} a=\bar{N}_{f} a=0$,

$$
\left(N_{f}-\frac{1}{2}\right) a=0
$$

$$
\mathcal{G}(z, z)=q(z, z)=0
$$

The first case is ruled out, because we must have $q(z, z)=-1$, in contradiction with the fact that $q$ is an even form.

In the second case, we must have $z=0$. Then from the formulas for $L_{0}, \bar{L}_{0}$ we see that such a vector has eigenvalues $(0,1 / 2)$ rather than $(1 / 2,0)$. Hence this case is also ruled out.

In the third case, we must have $z=0$. Furthermore, it is easy to see that all vectors satisfying (29) must also satisfy

$$
\begin{array}{ll}
\alpha_{s}^{i} a=\bar{\alpha}_{s}^{i} a=0, & i=1, \ldots, 2 d, s=1,2, \ldots, \\
\bar{\psi}_{r}^{i} a=0, & i=1, \ldots, 2 d, r=1 / 2,3 / 2, \ldots, \\
\psi_{r}^{i} a=0, & i=1, \ldots, 2 d, r=3 / 2,5 / 2, \ldots
\end{array}
$$

It follows that a must have the form

$$
a=\left(\sum_{i=1}^{2 d} c_{i} \psi_{-1 / 2}^{i}\right)|v a c, 0,0\rangle,
$$

where $c_{i}, i=1, \ldots, 2 d$, are arbitrary complex numbers. A similar argument shows that all eigenvectors of $L_{0}, \bar{L}_{0}$ with eigenvalues $(0,1 / 2)$ have the form

$$
\left(\sum_{i=1}^{2 d} \bar{c}_{i} \bar{\psi}_{-1 / 2}^{i}\right)|v a c, 0,0\rangle,
$$

where $\bar{c}_{i}, i=1, \ldots, 2 d$, are arbitrary complex numbers.

Now recall that $L_{0}^{\prime} f=f L_{0}$ and $\bar{L}_{0}^{\prime} f=f \bar{L}_{0}$. This implies that $f$ identifies the $(1 / 2,0)$ eigenspace of $\left(L_{0}, \bar{L}_{0}\right)$ with the $(1 / 2,0)$ eigenspace of $\left(L_{0}^{\prime}, \bar{L}_{0}^{\prime}\right)$, and $(0,1 / 2)$ eigenspace of $\left(L_{0}, \bar{L}_{0}\right)$ with the $(0,1 / 2)$ eigenspace of $\left(L_{0}^{\prime}, \bar{L}_{0}^{\prime}\right)$. Thus there exist two 
invertible complex matrices $F_{j}^{i}$ and $\bar{F}_{j}^{i}$ such that

$$
\begin{aligned}
& \psi_{-1 / 2}^{\prime i}|v a c, 0,0\rangle=f\left(F_{j}^{i} \psi_{-1 / 2}^{j}|v a c, 0,0\rangle\right), \\
& \bar{\psi}_{-1 / 2}^{\prime i}|v a c, 0,0\rangle=f\left(\bar{F}_{j}^{i} \bar{\psi}_{-1 / 2}^{j}|v a c, 0,0\rangle\right) .
\end{aligned}
$$

Applying $Y^{\prime}$ to both sides of this equation and using (26), we obtain:

$$
\psi^{\prime i}(z)=f F_{j}^{i} \psi^{j}(z) f^{-1}, \quad \bar{\psi}^{i}(\bar{z})=f \bar{F}_{j}^{i} \bar{\psi}^{j}(\bar{z}) f^{-1} .
$$

An immediate consequence of this is the transformation law for fermionic oscillators:

$$
\psi_{r}^{\prime i}=f F_{j}^{i} \psi_{r}^{j} f^{-1}, \quad \bar{\psi}_{r}^{\prime i}=f \bar{F}_{j}^{i} \bar{\psi}_{r}^{j} f^{-1}, \quad r \in \mathbb{Z}+\frac{1}{2} .
$$

Compatibility with the commutation relations of the fermionic oscillators then requires:

$$
F^{T} G^{\prime} F=G, \quad \bar{F}^{T} G^{\prime} \bar{F}=G .
$$

(Alternatively, one may derive this by comparing the OPE of $\psi(z), \bar{\psi}(\bar{z})$ with themselves and the OPE of $\psi^{\prime}(z), \bar{\psi}^{\prime}(\bar{z})$ with themselves.)

Now let us turn to bosonic oscillators. Consider the OPE of $Q(z)$ with $\psi(z)$ :

$$
Q(z) \psi^{i}(w) \sim \frac{i}{2 \sqrt{2}} \frac{\partial X^{i}(w)}{(z-w)} .
$$

Since $f$ preserves the OPE and takes $Q(z)$ to $Q^{\prime}(z)$, and $\psi(w)$ to $F^{-1} \psi^{\prime}(w)$, we infer that

$$
\partial X^{\prime i}(z)=f F_{j}^{i} \partial X^{j}(z) f^{-1} .
$$

Similarly, the OPE of $\bar{Q}(\bar{z})$ with $\bar{\psi}(w)$ implies that

$$
\bar{\partial} \bar{X}^{\prime i}(\bar{z})=f \bar{F}_{j}^{i} \bar{\partial} \bar{X}^{j}(\bar{z}) f^{-1}
$$

These formulas imply the following transformation laws for bosonic oscillators:

$$
\alpha_{n}^{\prime i}=f F_{j}^{i} \alpha_{n}^{j} f^{-1}, \quad \bar{\alpha}_{n}^{i}=f \bar{F}_{j}^{i} \bar{\alpha}_{n}^{j} f^{-1}, \quad n \in \mathbb{Z} .
$$

Another consequence is the transformation law of $Z$ :

$$
Z^{\prime}=f g Z f^{-1}
$$

where $g \in \operatorname{Hom}_{\mathbb{R}}\left(U \oplus U^{*}, U^{\prime} \oplus U^{\prime *}\right)$ is defined by

$$
g=R\left(G^{\prime}, B^{\prime}\right)^{-1}\left(\begin{array}{cc}
F & 0 \\
0 & \bar{F}
\end{array}\right) R(G, B),
$$


and $Z$ and $Z^{\prime}$ are regarded as elements of $\operatorname{End}\left(\mathbb{C}\left[\Gamma \oplus \Gamma^{*}\right]\right) \otimes_{\mathbb{R}}\left(U \oplus U^{*}\right)$ and $\operatorname{End}\left(\mathbb{C}\left[\Gamma^{\prime} \oplus\right.\right.$ $\left.\left.\Gamma^{\prime *}\right]\right) \otimes_{\mathbb{R}}\left(U^{\prime} \oplus U^{\prime *}\right)$. Now note that $Z$ and $Z^{\prime}$ are in fact "realifications" of some elements in $\operatorname{End}\left(\mathbb{C}\left[\Gamma \oplus \Gamma^{*}\right]\right) \otimes_{\mathbb{Z}}\left(\Gamma \oplus \Gamma^{*}\right)$ and $\operatorname{End}\left(\mathbb{C}\left[\Gamma^{\prime} \oplus \Gamma^{* *}\right]\right) \otimes_{\mathbb{Z}}\left(\Gamma^{\prime} \oplus \Gamma^{\prime *}\right)$. This means that $g$ is a "realification" of an element of $\operatorname{Hom}_{\mathbb{Z}}\left(\Gamma \oplus \Gamma^{*}, \Gamma^{\prime} \oplus \Gamma^{\prime *}\right)$, which we also denote $g$.

It remains to show that $g$ takes $q$ to $q^{\prime}$ and $\mathcal{G}$ to $\mathcal{G}^{\prime}$. To this end notice that the transformation laws for the oscillators imply

$$
N_{b}^{\prime}=f N_{b} f^{-1}, \quad N_{f}^{\prime}=f N_{f} f^{-1}, \quad \bar{N}_{b}^{\prime}=f \bar{N}_{b} f^{-1}, \quad \bar{N}_{f}^{\prime}=f \bar{N}_{f} f^{-1}
$$

Then it follows from $L_{0}^{\prime}=f L_{0} f^{-1}$ and $\bar{L}_{0}^{\prime}=f \bar{L}_{0} f^{-1}$ that for all $x \in \Gamma \oplus \Gamma^{*}$ we have

$$
\begin{aligned}
q^{\prime}(g x, g x) & =q(x, x), \\
\mathcal{G}^{\prime}(g x, g x) & =\mathcal{G}(x, x) .
\end{aligned}
$$

This concludes the proof of the theorem.

\subsection{Isomorphisms of $N=2$ SCVA's}

The goal of this subsection is to prove Theorem 2.1 which we restate below. Given a metric $G$ on $U$, a compatible complex structure $I$ on $U$, and $B \in \Lambda^{2} U^{*}$, we define a pair of commuting complex structures on $U \oplus U^{*}$ as follows:

$$
\begin{aligned}
\mathcal{I}(I, B) & =\left(\begin{array}{cc}
I & 0 \\
B I+I^{t} B & -I^{t}
\end{array}\right), \\
\mathcal{J}(G, I, B) & =\left(\begin{array}{cc}
-I G^{-1} B & I G^{-1} \\
G I-B I G^{-1} B & B I G^{-1}
\end{array}\right) .
\end{aligned}
$$

The complex structure $\mathcal{J}$ can be expressed in terms of the Kähler form $\omega=G I$ and $B$ :

$$
\mathcal{J}(\omega, B)=\left(\begin{array}{cc}
\omega^{-1} B & -\omega^{-1} \\
\omega+B \omega^{-1} B & -B \omega^{-1}
\end{array}\right) .
$$

We will use a simplified notation $\mathcal{I}(I, B)=\mathcal{I}, \quad \mathcal{I}\left(I^{\prime}, B^{\prime}\right)=\mathcal{I}^{\prime}$, etc. The complex structures $\mathcal{I}, \mathcal{J}$ and the symmetric forms $\mathcal{G}, q$ are related by an identity

$$
\mathcal{G}=-2 q \mathcal{I} \mathcal{J}
$$

where $\mathcal{G}$ and $q$ are understood as elements of $\operatorname{Hom}_{\mathbb{R}}\left(U, U^{*}\right)$.

Theorem $5.2 \operatorname{Vert}(\Gamma, I, G, B)$ is isomorphic to $\operatorname{Vert}\left(\Gamma^{\prime}, I^{\prime}, G^{\prime}, B^{\prime}\right)$ as an $N=2 S C V A$ if and only if there is an isomorphism of lattices $\Gamma \oplus \Gamma^{*}$ and $\Gamma^{\prime} \oplus \Gamma^{\prime *}$ which takes $q$ to $q^{\prime}, \quad \mathcal{I}$ to $\mathcal{I}^{\prime}$, and $\mathcal{J}$ to $\mathcal{J}^{\prime}$. 
To prove this theorem, note that $f: V \rightarrow V^{\prime}$ is an isomorphism of $N=2$ SCVA's if and only if it is an isomorphism of the underlying $N=1$ SCVA's, and maps $J(z)$ to $J^{\prime}(z)$ and $\bar{J}(\bar{z})$ to $\bar{J}^{\prime}(\bar{z})$. Now suppose $f$ is an isomorphism of $N=1$ SCVA's underlying $\operatorname{Vert}(\Gamma, I, G, B)$ and $\operatorname{Vert}\left(\Gamma^{\prime}, I^{\prime}, G^{\prime}, B^{\prime}\right)$. By Theorem 5.1 we know that there exists $g \in \operatorname{Hom}\left(\Gamma \oplus \Gamma^{*}, \Gamma^{\prime} \oplus \Gamma^{\prime *}\right)$ which takes $q$ to $q^{\prime}$, and $\mathcal{G}$ to $\mathcal{G}^{\prime}$. To prove the theorem, it is sufficient to show that $f$ maps $J(z), \bar{J}(\bar{z})$ correctly if and only if $g$ maps $\mathcal{I}$ to $\mathcal{I}^{\prime}$ and $\mathcal{J}$ to $\mathcal{J}^{\prime}$. In fact, since $\mathcal{G}=-2 q \mathcal{I} \mathcal{J}$ and $\mathcal{G}^{\prime}=-2 q^{\prime} \mathcal{I}^{\prime} \mathcal{J}^{\prime}$, it is sufficient to show that $f$ maps $J(z), \bar{J}(\bar{z})$ correctly if and only if $g$ maps $\mathcal{I}$ to $\mathcal{I}^{\prime}$.

Using the transformation law (27) for the fields and the formula (25) relating $G$ and $G^{\prime}$, one can easily see that $f$ maps $J(z)$ to $J^{\prime}(z)$ if and only if

$$
I^{\prime}=\left(a-b H^{t}\right) I\left(a-b H^{t}\right)^{-1}
$$

Similarly, $f$ maps $\bar{J}(\bar{z})$ to $\bar{J}^{\prime}(\bar{z})$ if and only if

$$
I^{\prime}=(a+b H) I(a+b H)^{-1} .
$$

On the other hand, $\mathcal{I}(I, B)$ can be written as

$$
\mathcal{I}(I, B)=R(G, B)^{-1}\left(\begin{array}{ll}
I & 0 \\
0 & I
\end{array}\right) R(G, B) .
$$

This and the identity (23) imply that $\mathcal{I}^{\prime}=g \mathcal{I} g^{-1}$ if and only if

$$
\left(\begin{array}{cc}
I^{\prime} & 0 \\
0 & I^{\prime}
\end{array}\right)=\mathcal{M}(g, H)\left(\begin{array}{ll}
I & 0 \\
0 & I
\end{array}\right) \mathcal{M}(g, H)^{-1} .
$$

This matrix identity is equivalent to (30,31), which proves the theorem.

Let us also note the following simple corollary of this theorem.

Corollary 5.3 Let $(T, I, G, B)$ be a complex torus equipped with a flat Kähler metric and a $B$-field of type $(1,1)$. Let $T^{\prime}=U^{\prime} / \Gamma^{\prime}$ be another torus of the same dimension and $I^{\prime}$ be a complex structure on $T^{\prime}$. Let $\tilde{\mathcal{I}}$ and $\tilde{\mathcal{I}}^{\prime}$ be the product complex structures on $T \times T^{*}$ and $T^{\prime} \times T^{*}$. Suppose there exists an isomorphism of lattices $g: \Gamma \oplus \Gamma^{*} \rightarrow \Gamma^{\prime} \oplus \Gamma^{*}$ mapping $q$ to $q^{\prime}$ and $\tilde{\mathcal{I}}$ to $\tilde{\mathcal{I}}^{\prime}$. Then on $T^{\prime}$ there exists a Kähler metric $G^{\prime}$ and a B-field of type $(1,1)$ such that $\operatorname{Vert}(\Gamma, I, G, B)$ is isomorphic to $\operatorname{Vert}\left(\Gamma^{\prime}, I^{\prime}, G^{\prime}, B^{\prime}\right)$ as an $N=2$ SCVA.

To show this, we define $H^{\prime}$ using (22) and set $G^{\prime}$ and $B^{\prime}$ to be the symmetric and skewsymmetric parts of $H^{\prime}$, respectively. Then it follows from (25) that $G^{\prime}$ is positive-definite. By Theorem 5.1 the $N=1$ SCVA corresponding to $(T, G, B)$ is isomorphic to $N=1$ 
SCVA corresponding to $\left(T^{\prime}, G^{\prime}, B^{\prime}\right)$. Using that fact that $g$ intertwines $\tilde{\mathcal{I}}$ to $\tilde{\mathcal{I}}^{\prime}$ it is easy to show that $H^{\prime} I^{\prime}+I^{\prime} H^{\prime}=0$, which means that $G^{\prime}$ is a Kähler metric and $B^{\prime}$ has type $(1,1)$. In particular, $\tilde{\mathcal{I}}^{\prime}=\mathcal{I}^{\prime}$. Then it follows from the identity $\mathcal{G}^{\prime}=-2 q^{\prime} \mathcal{I}^{\prime} \mathcal{J}^{\prime}$ and the fact $g$ intertwines $\mathcal{G}, q, \mathcal{I}$ and $\mathcal{G}^{\prime}, q^{\prime}, \mathcal{I}^{\prime}$ that $g$ also intertwines $\mathcal{J}$ and $\mathcal{J}^{\prime}$. Theorem 5.2 then implies that $\operatorname{Vert}(\Gamma, I, G, B)$ is isomorphic to $\operatorname{Vert}\left(\Gamma^{\prime}, I^{\prime}, G^{\prime}, B^{\prime}\right)$ as an $N=2$ SCVA.

\subsection{Mirror morphisms of $N=2$ SCVA's}

In this subsection we establish a criterion for the existence of a mirror morphism between two complex tori equipped with flat Kähler metrics and B-fields.

Theorem 5.4 $\operatorname{Vert}(\Gamma, I, G, B)$ is mirror to $\operatorname{Vert}\left(\Gamma^{\prime}, I^{\prime}, G^{\prime}, B^{\prime}\right)$ if and only if there is an isomorphism of lattices $\Gamma \oplus \Gamma^{*}$ and $\Gamma^{\prime} \oplus \Gamma^{\prime *}$ which takes $q$ to $q^{\prime}, \mathcal{I}$ to $\mathcal{J}^{\prime}$, and $\mathcal{J}$ to $\mathcal{I}^{\prime}$.

The proof is very similar to that of Theorem 5.2. Again it is sufficient to show that if $f$ is an isomorphism of the underlying $N=1$ SCVA's, and $g$ the corresponding isomorphism of lattices, then

$$
f J(z) f^{-1}=-J^{\prime}(z), \quad f \bar{J}(\bar{z}) f^{-1}=\bar{J}^{\prime}(\bar{z})
$$

is equivalent to

$$
g \mathcal{J} g^{-1}=\mathcal{I}^{\prime}
$$

The first of these is equivalent to

$$
I^{\prime}=(a+b H) I(a+b H)^{-1}=-\left(a-b H^{t}\right) I\left(a-b H^{t}\right)^{-1}
$$

On the other hand, $\mathcal{J}(G, I, B)$ can be written as

$$
\mathcal{J}(G, I, B)=R(G, B)^{-1}\left(\begin{array}{cc}
-I & 0 \\
0 & I
\end{array}\right) R(G, B),
$$

which together with (23) and (32) implies that (33) is equivalent to

$$
\mathcal{M}(g, H)\left(\begin{array}{cc}
-I & 0 \\
0 & I
\end{array}\right) \mathcal{M}(g, H)^{-1}=\left(\begin{array}{cc}
I^{\prime} & 0 \\
0 & I^{\prime}
\end{array}\right) .
$$

This is obviously equivalent to (34). This concludes the proof. 


\section{Homological mirror symmetry with B-fields}

\subsection{Mirror symmetry and D-branes}

As explained in Section 2, Kontsevich's conjecture must be modified if the B-field does not vanish. When the image of $\mathcal{B}$ in $H^{2}\left(X, \mathcal{O}_{X}^{*}\right)$ is torsion, our results on complex tori suggest that the bounded derived category $D^{b}(X)$ should be replaced with $D^{b}(X, \mathcal{B})$, the bounded derived category of coherent modules over an Azumaya algebra. The similarity class of the Azumaya algebra is determined by the image of $\mathcal{B}$ in $H^{2}\left(X, \mathcal{O}_{X}^{*}\right)$. (Presumably, when $\mathcal{B}$ does not map to a torsion class, the proper analogue of $D^{b}(X)$ is some "coherent" subcategory of the derived category of quasicoherent sheaves on a gerbe over $X$, see Remark 2.6.) However, this does not provide any hint as to what the modification of the Fukaya category might be. In this section we explain some string theory lore which suggests a particular definition of the Fukaya category in the presence of the B-field. A similar proposal has been made in [2].

The ordinary $\sigma$-model whose quantization yields an $N=2$ superconformal vertex algebra is a classical field theory on a two-dimensional manifold $\Sigma=\mathbb{R} \times \mathbb{S}^{1}$ ("the worldsheet"). Let us replace $\mathbb{S}^{1}$ with an interval $I=[0,1]$ and consider the same $\sigma$-model on a worldsheet with boundaries $\mathbb{R} \times I$. This procedure is referred to as passing from closed to open strings. Now, in order to make the space of solutions of the Euler-Lagrange equations a symplectic supermanifold, one has to supply boundary conditions for the fields of the $\sigma$ model on both ends of the interval. In addition one requires that these boundary conditions preserve $N=2$ superconformal symmetry. To be more precise, while the classical $\sigma$-model on $\mathbb{R} \times \mathbb{S}^{1}$ has two copies of the $N=2$ super-Virasoro algebra (with zero central charge) as its classical symmetry, the $\sigma$-model on $\mathbb{R} \times I$ is required to be symmetric only with respect to a single $N=2$ super-Virasoro algebra. There are two essentially different classes of such boundary conditions, called A and B boundary conditions. The B-type boundary conditions preserve the "diagonal" super-Virasoro subalgebra whose generators are given by

$$
L_{n}+\bar{L}_{n}, \quad J_{n}+\bar{J}_{n}, \quad Q_{r}^{+}+\bar{Q}_{r}^{+}, \quad Q_{r}^{-}+\bar{Q}_{r}^{-}, \quad n \in \mathbb{Z}, r \in \mathbb{Z}+\frac{1}{2} .
$$

The A-type boundary conditions preserve a different subalgebra whose generators are

$$
L_{n}+\bar{L}_{n}, \quad-J_{n}+\bar{J}_{n}, \quad Q_{r}^{-}+\bar{Q}_{r}^{+}, \quad Q_{r}^{+}+\bar{Q}_{r}^{-}, \quad n \in \mathbb{Z}, r \in \mathbb{Z}+\frac{1}{2} .
$$

Superconformally-invariant boundary conditions for a $\sigma$-model are called supersymmetric (or BPS, for Bogomolny-Prasad-Sommerfeld) D-branes. Thus we have BPS D-branes of types A and B. Note that the mirror involution (2) exchanges the two types of D-branes.

D-branes are understood best when the B-field is zero. In this case one can construct examples of the B-type boundary conditions by starting from a holomorphic submanifold of 
the Calabi-Yau manifold $X$. More generally, one can start from a holomorphic submanifold $M \subset X$ and a holomorphic bundle on $M$ equipped with a compatible connection. On the other hand, examples of the A-type boundary conditions (with zero B-field) can be constructed starting from a Lagrangian submanifold $L \subset X$ (with respect to the Kähler form), a trivial unitary bundle $E$ on $L$, and a unitary flat connection on $E$.

Note that one can choose different boundary conditions for the Euler-Lagrange equations on the two ends of the interval $I$. The only constraint is that both boundary conditions must be of the same type (A or B). If this condition is violated, then the symmetry of the corresponding classical field theory is only some subalgebra of the $N=2$ super-Virasoro algebra, namely an $N=1$ super-Virasoro algebra.

After quantization, $\sigma$-model on $\mathbb{R} \times I$ is supposed to yield a superconformally invariant quantum field theory on the same manifold. The axioms of such quantum field theories have not been formulated yet, and we will not attempt it here. Suffice it to say that physicists expect that any B-type D-brane can be consistently quantized, while A-type boundary conditions may lead to "anomalies," i.e. inconsistencies in the quantization procedure. One can argue that anomalies are absent if the A-type D-brane originates from a special Lagrangian submanifold. We remind that a special Lagrangian submanifold in a Calabi-Yau manifold with a Kähler metric is defined by two properties: it is Lagrangian, and the restriction of a nonzero section of the canonical bundle to the submanifold is proportional to its volume form.

Thus to any physicist's Calabi-Yau with zero B-field one can associate two sets: the set of B-type D-branes, and the set of (non-anomalous) A-type D-branes. The former set has many elements in common with the set of coherent sheaves on $X$. The latter set resembles the set of objects the Fukaya category of $X$. Moreover, there are heuristic arguments using path integrals showing that either A or B-type D-branes form an $A_{\infty}$-category (see [16] and references therein). Thus, conjecturally, to every physicist's Calabi-Yau with zero B-field one can canonically associate a pair of $A_{\infty}$-categories, the categories of A- and B-type D-branes. Assuming there are shift functors on them, one can define the corresponding triangulated categories as in [23].

It is natural to conjecture that for $\mathcal{B}=0$ the triangulated category associated with A-type (resp. B-type) D-branes is equivalent to $D \mathcal{F}(X)$ (resp. $D^{b}(X)$ ) [32, 10]. There are several pieces of evidence supporting this conjecture. First, as we have already remarked, $\mathcal{F}(X)$ and $\operatorname{Coh}(X)$ have many objects in common with the categories of $\mathrm{A}$ and B-type D-branes, respectively. Second, using path integrals one can argue [33] that the category of B-type D-branes is independent of the Kähler form, while the category of A-type D-branes is independent of the complex structure on $X$ if $\omega$ is fixed. For further evidence see 10 
and references therein.

If this conjecture is true, then Kontsevich's conjecture has a natural explanation. Suppose we have a mirror pair of physicist's Calabi-Yaus $X$ and $X^{\prime}$, both with zero B-field. The corresponding $N=2$ SCVA's are related by a mirror morphism. Since a mirror morphism of $N=2$ SCVA's acts on the $N=2$ super-Virasoro by the mirror involution, it exchanges the A and B-type boundary conditions. Hence it induces an equivalence of $D^{b}(X)$ with the derived Fukaya category $D \mathcal{F}_{0}\left(X^{\prime}\right)$, and vice versa.

\subsection{Fukaya category with a B-field}

Now let us generalize this to nonzero B-fields. We already know the effect of a B-field on $D^{b}(X)$ : the sheaf $\mathcal{O}_{X}$ is replaced with a certain sheaf of noncommutative algebras. This agrees with the string theory lore that the B-field makes the D-brane worldvolume noncommutative [0, 11].

The effect of the B-field on the Fukaya category seems rather different. Let us start by recalling the definition of the set of objects of the Fukaya category [23]. Let $(X, \omega)$ be a symplectic manifold of dimension $2 d$. We fix an almost complex structure $I$ on $X$ compatible with $\omega$ and thereby obtain a Hermitian metric on $X$. (If $X$ is a physicist's Calabi-Yau, it automatically comes equipped with a compatible complex structure). Moreover, we assume that $c_{1}\left(T_{X}^{h o l}\right)=0$. In this case the line bundle $\Lambda^{d}\left(\Omega_{X}^{h o l}\right)$ is trivial and has a nowhere vanishing holomorphic section $\Omega$ which is called a calibration.

Naively, an object of the Fukaya category should be a triple $(L, E, \nabla)$, where $L$ is a Lagrangian submanifold, $E$ is a trivial unitary vector bundle on $L$, and $\nabla$ is a flat connection on $E$. From the physical point of view, such a triple allows one to define an A-type boundary condition for the classical $\sigma$-model, and therefore it is an A-type Dbrane [33, 26].

The naive definition of an object does not allow one to define a nontrivial shift functor and $A_{\infty}$ structure. This difficulty can be overcome as follows [23]. For any point $x \in L$ the tangent space $T_{x} L$ is a Lagrangian subspace of $T_{x} X$. The Grassmannian of Lagrangian subspaces has fundamental group equal to $\mathbb{Z}$. Each Lagrangian submanifold comes with a Gauss map from $L$ to $\mathcal{L} G$, where $\mathcal{L} G \rightarrow X$ is a fibration whose fiber over $x$ is the Grassmannian of Lagrangian subspaces of $T_{x} X$. Consider a fibration $\widetilde{\mathcal{L} G} \rightarrow X$ covering $\mathcal{L} G \rightarrow X$ such that its fiber is the universal cover of the fiber of $\mathcal{L} G \rightarrow X$. (As mentioned in [23], there is a canonical choice of such a fibration if $c_{1}\left(T_{X}^{h o l}\right)=0$. ) Instead of $L$, we will consider pairs $(L, i)$, where $i$ is a lift of the Gauss map to $\widetilde{\mathcal{L} G}$. Not every Lagrangian $L$ admits such a lift, so not any Lagrangian submanifold can be extended to an object of the Fukaya category. Note that any Lagrangian $L$ comes equipped with two natural $d$-forms: 
the volume form and the restriction of the calibration $\Omega$. The latter is defined up to a multiplicative constant. Their quotient is a nowhere vanishing function $f$ which maps $L$ to $\mathbb{C}^{*}$. One can show that the Gauss map admits a lift to $\widetilde{\mathcal{L} G}$ if and only if the image $f(L)$ is contractible. For example, any special Lagrangian $L$ has a lift, because by definition of speciality the function $f$ is constant for any such $L$.

To summarize, we can define an object of the Fukaya category in the absence of the B-field as a quadruple $(L, i, E, \nabla)$, where $L$ and $i$ are as above, and $(E, \nabla)$ is a trivial complex vector bundle on $L$ with a unitary flat connection. The natural fiberwise action of $\mathbb{Z}$ on $\widetilde{\mathcal{L} G} \rightarrow X$ induces an action of $\mathbb{Z}$ on such quadruples. One hopes that this action extends to a shift functor from the Fukaya category to itself.

Now let us try to guess how the definition of the Fukaya category should be modified when $\mathcal{B} \neq 0$. Let $B$ be a closed 2 -form on $X$ representing $\mathcal{B} \in H^{2}(X, \mathbb{R} / \mathbb{Z})$. (Since we assumed that $\mathcal{B}$ is in the kernel of the Bockstein homomorphism $H^{2}(X, \mathbb{R} / \mathbb{Z}) \rightarrow H^{3}(X, \mathbb{Z})$, such a 2-form exists.) Let $F_{\nabla}$ be the curvature of a connection $\nabla$ on a bundle $E$ on $L$. If $B=0$, the condition on $\nabla$ is

$$
F_{\nabla}=0
$$

On the other hand, it is a general principle of string theory that the equations of motion must be invariant with respect to a substitution

$$
B \rightarrow B+d \lambda, \quad \nabla \rightarrow \nabla+\left.2 \pi i i d_{E} \lambda\right|_{L},
$$

where $\lambda$ is any real 1-form on $X$. This must be true because the action of the $\sigma$-model on $\mathbb{R} \times I$ is invariant with respect to such transformations [29]. This requirement is sufficient to fix the generalization of (35) to arbitrary $B$ :

$$
F_{\nabla}=\left.2 \pi i i d_{E} B\right|_{L} .
$$

We propose that an object of the Fukaya category for $\mathcal{B} \neq 0$ is a quadruple $(L, i, E, \nabla)$, where $L$ and $i$ are the same as above, $E$ is a complex vector bundle on $L$, and $\nabla$ is a connection on $E$ satisfying (37).

We can make some checks of this proposal. First, our definition of an object depends on how one lifts $\mathcal{B} \in H^{2}(X, \mathbb{R} / \mathbb{Z})$ to a 2 -form $B$. However, given two different 2-forms $B_{1}$ and $B_{2}$ representing $\mathcal{B}$, there is a one-to-one map between the corresponding sets of objects. Indeed, let $f=B_{2}-B_{1}$. It is easy to see that $f$ has integral periods, and therefore there exists a line bundle $\mathcal{N}$ on $X$ and a connection $\nabla_{0}$ on $\mathcal{N}$ such that the curvature of $\nabla_{0}$ is equal to $2 \pi i f$. The bijection between the set of objects corresponding to $B_{1}$ and the set of objects corresponding to $B_{2}$ is given by

$$
L \mapsto L, \quad i \mapsto i,\left.\quad E \mapsto E \otimes \mathcal{N}\right|_{L}, \quad \nabla_{1} \mapsto \nabla_{1} \otimes i d_{\mathcal{N}}+i d_{E} \otimes \nabla_{0} .
$$


Second, from the equation (37) we see that $c_{1}(E)=\left.\operatorname{rank}(E) b\right|_{L}$, where $b$ is the de Rham cohomology class of $B$. Since $c_{1}(E)$ is integral, we infer that

$$
\left.\operatorname{rank}(E) \mathcal{B}\right|_{L}=0
$$

In particular, for $\operatorname{rank}(E)=1$, we get that the restriction of $\mathcal{B}$ to $L$ must vanish. This is consistent with the results of Hori et al. [18], who analyzed the A-type boundary conditions in the rank-one case. Hori et al. find that the restriction of $B$ to $L$ must be zero if one wants to make an A-type D-brane out of $L$. We found that it is sufficient to require $\left.\mathcal{B}\right|_{L}=0$.

We need to address one more subtlety. The original HMSC required $E$ to be a unitary vector bundle and $\nabla$ to be a unitary connection [23]. This requirement naturally arises in the string theory context as well. Nevertheless, this condition is much too strong. Even in the case of the elliptic curve one has to allow for non-unitary connections on the A-side if one wants to account for all bundles on the B-side [31]. In that case, the right thing to do is to require the holonomy representation of $\nabla$ to have eigenvalues with unit modulus. It is natural to conjecture that this is also the right requirement for $\operatorname{dim}_{\mathbb{C}} X>1$ or $\mathcal{B} \neq 0$.

In the absence of the B-field, any pair $(L, i)$ can be extended (in many different ways) to an object of the Fukaya category. The situation is more complex for $\mathcal{B} \neq 0$. Recall that to any flat connection on a manifold $L$ one can canonically associate a finite-dimensional representation of $\pi_{1}(L)$ (or, equivalently, a finite-dimensional representation of the group algebra of $\left.\pi_{1}(L)\right)$, and vice versa. In fact, this map is a one-to-one correspondence. Similarly, given a bundle $E$ on $L$ and a connection $\nabla$ on $E$ such that $F_{\nabla}$ satisfies (37), one can construct a finite-dimensional representation of a twisted group algebra of $\pi_{1}(L)$ in the following way. To $(E, \nabla)$ we can associate a projective representation $R$ of $\pi_{1}(L)$. To any such $R$ one can attach an element $\psi_{R}$ of $H^{2}\left(\pi_{1}(L), U(1)\right)$. Acting on it with the natural embedding

$$
H^{2}\left(\pi_{1}(L), U(1)\right) \stackrel{j}{\rightarrow} H^{2}(L, U(1))
$$

we obtain an element $j\left(\psi_{R}\right) \in H^{2}(L, U(1))$. One can show that $j\left(\psi_{R}\right)=\left.\mathcal{B}\right|_{L}$ (we identify $\mathbb{R} / \mathbb{Z}$ with $U(1))$.

To any 2-cocycle $\psi$ one can associate a twisted group algebra $\mathbb{C}_{\psi}\left[\pi_{1}(L)\right]$, which is a vector space generated by the elements of $\pi_{1}(L)$ with the following multiplication law:

$$
g \cdot h=\psi(g, h) g h, \quad g, h \in \pi_{1}(L) .
$$

The correspondence between pairs $(E, \nabla)$ satisfying (37) and finite-dimensional representations of the twisted group algebra $\mathbb{C}_{\psi}\left[\pi_{1}(L)\right]$ is one-to-one. A proof of this fact is given 
in Appendix 9. The eigenvalues of the holonomy representation of $\nabla$ have unit modulus if an only if the eigenvalues of $g \in \pi_{1}(L)$ have unit modulus. In particular this means that a Lagrangian submanifold $L$ can be extended to an object of the Fukaya category only if $\left.\mathcal{B}\right|_{L}$ is in the image of the homomorphism (39).

As a by-product, we obtained an equivalent definition of an object of the Fukaya category: it is a triple $(L, i, R)$, where $L, i$ are the same as above, and $R$ is a finite-dimensional representation of the twisted group algebra $\mathbb{C}_{\psi}\left[\pi_{1}(L)\right]$ such that $j\left(\psi_{R}\right)=\left.\mathcal{B}\right|_{L}$ and all the eigenvalues of $R(g)$ have unit modulus for all $g \in \pi_{1}(L)$.

Morphisms in the modified Fukaya category $\mathcal{F}(X, \mathcal{B})$ are defined in analogy with [12, 23. Let $\mathcal{U}_{1}=\left(L_{1}, i_{1}, E_{1}, \nabla_{1}\right)$ and $\mathcal{U}_{2}=\left(L_{2}, i_{2}, E_{2}, \nabla_{2}\right)$ be two objects such that $L_{1}$ and $L_{2}$ intersect transversally. Morphisms from $\mathcal{U}_{1}$ to $\mathcal{U}_{2}$ in $\mathcal{F}(X)$ form a complex of vector spaces defined by the rule

$$
\operatorname{Hom} \cdot\left(\mathcal{U}_{1}, \mathcal{U}_{2}\right)=\bigoplus_{x \in L_{1} \cap L_{2}} \operatorname{Hom}^{i}\left(\left.E_{1}\right|_{x},\left.E_{2}\right|_{x}\right)
$$

It is graded in the following way. For any point $x \in L_{1} \cap L_{2}$ we have two points $i_{1}(x)$ and $i_{2}(x)$ on the universal cover of the Lagrangian Grassmannian of $T_{x} X$. To these two points we can associate an integer $\mu\left(i_{1}(x), i_{2}(x)\right)$ which is called the Maslov index of $i_{1}(x), i_{2}(x)$ (see for example [3]). By definition, the space $\operatorname{Hom}\left(\left.E_{1}\right|_{x},\left.E_{2}\right|_{x}\right)$ has a grading $\mu\left(i_{1}(x), i_{2}(x)\right)$.

The differential on $\operatorname{Hom}\left(\mathcal{U}_{1}, \mathcal{U}_{2}\right)$ is defined by the rule

$$
d(u)=\sum_{z \in L_{1} \cap L_{2}} m_{1}(u ; z),
$$

where $u \in \operatorname{Hom}\left(\left.E_{1}\right|_{x},\left.E_{2}\right|_{x}\right)$, and $m_{1}(u ; z) \in \operatorname{Hom}\left(\left.E_{1}\right|_{z},\left.E_{2}\right|_{z}\right)$ is given by

$$
m_{1}(u ; z)=\sum_{\phi: D \rightarrow X} \pm \exp \left(2 \pi i \int_{D} \phi^{*}(-B+i \omega)\right) \cdot P \exp \left(\oint_{\partial D} \phi^{*} \nabla\right) .
$$

Here $\phi$ is an (anti)-holomorphic map from the disk $D=\{|w| \leq 1, w \in \mathbb{C}\}$ to $X$ such that $\phi(-1)=x, \phi(1)=z$ and $\phi([x, z]) \subset L_{2}$ and $\phi([z, x]) \subset L_{1}$. The path-ordered integral is defined by the following rule

$$
P \exp \left(\oint_{\partial D} \phi^{*} \nabla\right):=P \exp \left(\int_{x}^{z} \phi^{*} \nabla_{2}\right) \cdot u \cdot P \exp \left(\int_{z}^{x} \phi^{*} \nabla_{1}\right)
$$

This homomorphism from $\left.E_{1}\right|_{z}$ to $\left.E_{2}\right|_{z}$ can be described as follows. We take a vector $\left.e \in E_{1}\right|_{z}$, use the connection $\nabla_{1}$ transport it to $\left.E_{1}\right|_{x}$, apply the map $u$, and obtain an element of $\left.E_{2}\right|_{x}$. Then we transport this element to $\left.E_{2}\right|_{z}$ using the connection $\nabla_{2}$.

The \pm sign indicates the natural orientation on the space of (anti)-holomorphic maps. One expects that there are finitely many such maps if $\mu_{z}-\mu_{x}=1$. 
To define the composition of morphisms, let us take $u \in \operatorname{Hom}\left(\left.E_{1}\right|_{x},\left.E_{2}\right|_{x}\right)$ and $v \in$ $\operatorname{Hom}\left(\left.E_{2}\right|_{y},\left.E_{3}\right|_{y}\right)$, where $x \in L_{1} \cap L_{2}$ and $y \in L_{2} \cap L_{3}$. Then the composition of $u$ and $v$ is defined as

$$
v \circ u=\sum_{z \in L_{1} \cap L_{3}} m_{2}(v, u ; z)
$$

where $m_{2}(v, u ; z) \in \operatorname{Hom}\left(\left.E_{1}\right|_{z},\left.E_{3}\right|_{z}\right)$ is given by

$$
m_{2}(v, u ; z)=\sum_{\phi: D \rightarrow X} \pm \exp \left(2 \pi i \int_{D} \phi^{*}(-B+i \omega)\right) \cdot P \exp \left(\oint_{\partial D} \phi^{*} \nabla\right)
$$

Here we sum over (anti)-holomorphic maps $\phi$ from a two-dimensional disk $D$ to $X$, such that three fixed points $p_{1}, p_{2}, p_{3} \in \partial D$ are mapped to $x, y, z$ respectively, and $\phi\left(\left[p_{i}, p_{i+1}\right]\right) \in L_{i+1}$. The path-ordered integral here is calculated by the rule

$$
P \exp \left(\oint_{\partial D} \phi^{*} \nabla\right):=P \exp \left(\int_{p_{2}}^{p_{3}} \phi^{*} \nabla_{3}\right) \cdot v \cdot P \exp \left(\int_{p_{1}}^{p_{2}} \phi^{*} \nabla_{2}\right) \cdot u \cdot P \exp \left(\int_{p_{3}}^{p_{1}} \phi^{*} \nabla_{1}\right)
$$

In the same manner we can define higher order compositions using zero-dimensional components of spaces of maps $\phi$ from the disk $D$ to $X$ with $\phi(\partial D)$ sitting in the union of Lagrangian submanifolds.

It is easy to check that the above definition of morphisms and their compositions does not change if we replace $B$ with another 2 -form with the same image in $H^{2}(X, \mathbb{R} / \mathbb{Z})$. The check makes use of (37) and (38). This confirms our claim that the Fukaya category depends only on $\mathcal{B}$.

The rules for computing morphisms and their compositions can be explained heuristically using the path integral for the $\sigma$-model on a worldsheet with boundaries [33].

The category $\mathcal{F}_{0}(X)$ has the same objects as $\mathcal{F}(X)$, but the morphisms are the degree zero cohomology groups of the complexes defined above. Note that different objects of $\mathcal{F}(X)$ often become isomorphic in $\mathcal{F}_{0}(X)$. For example, in the case when $X$ is a real symplectic 2-torus, any one-dimensional submanifold is Lagrangian. Many of them admit a lift of the Gauss map. Thus the category $\mathcal{F}(X)$ contains many more objects than the derived category of the elliptic curve (an elliptic curve with a flat metric is self-mirror). But in $\mathcal{F}_{0}(X)$ any object becomes isomorphic to some other object associated with a special Lagrangian submanifold (see [31]). More generally, it appears likely that working in the category $\mathcal{F}_{0}(X)$ one may restrict the set of objects of the Fukaya category and consider only special Lagrangian submanifolds with respect to a holomorphic calibration. For different $L$ the calibrations may differ by a multiplicative constant. This restriction is also natural from the string theory point of view, since, as explained above, non-anomalous A-type Dbranes are associated with special Lagrangian submanifolds in a Calabi-Yau [26]. 


\section{A Supersymmetric $\sigma$-model of a flat torus}

In this section we define the classical field theory known in the physics literature as the $N=1$ supersymmetric $\sigma$-model. The data needed to specify a $\sigma$-model consist of a $C^{\infty}$ manifold $M$ ("the target space"), a Riemannian metric $G$ on $M$, and a 2-form $B$ on $M$. We then discuss the problem of the quantization of the $\sigma$-model in the case when the target space is a flat torus. The superconformal vertex algebra constructed in Section 1 can be regarded as a solution of the quantization problem. A detailed discussion of supersymmetric $\sigma$-models can be found in [8].

Let $\mathcal{W}$ be a two-dimensional $C^{\infty}$ manifold $\mathbb{R} \times \mathbb{S}^{1}$ ("the worldsheet"). We parametrize $\mathcal{W}$ by $(\tau, \sigma) \in \mathbb{R} \times \mathbb{R} /(2 \pi \mathbb{Z})$. The coordinate $\tau$ is regarded as "time." We endow $\mathcal{W}$ with a Minkowskian metric $d s^{2}=d \tau^{2}-d \sigma^{2}$ and orientation $d \tau \wedge d \sigma$. Thus $* d \sigma=$ $d \tau, * d \tau=d \sigma$. The symmetric tensor corresponding to the metric will be denoted $g$. General coordinates on $\mathcal{W}$ will be denoted $\left(y^{0}, y^{1}\right)$. The invariant volume element $d \tau \wedge d \sigma=$ $d^{2} y \sqrt{-\operatorname{det} g}$ will be denoted $d \Sigma$. We denote by $S^{+}$and $S^{-}=S^{+*}$ the complexified semispinor representations of $S O(1,1)$ and by $V$ its complexified fundamental representation. Complexified semi-spinor representations are one-dimensional complex vector spaces endowed with $S O(1,1)$-invariant nondegenerate morphisms

$$
\gamma: S^{-} \rightarrow V \otimes S^{+}, \quad \bar{\gamma}: S^{+} \rightarrow V \otimes S^{-}
$$

These morphisms are determined up to a scalar factor, and we assume that they satisfy the Clifford algebra relation

$$
\gamma \bar{\gamma}+\bar{\gamma} \gamma=2 g^{-1} \cdot \mathbf{i d}_{S^{+} \oplus S^{-}}
$$

Here $g^{-1}$ is regarded as map $\mathbb{C} \rightarrow V^{*} \otimes V^{*}$. In a suitable basis, one has

$$
\gamma=\left(\begin{array}{c}
1 \\
-1
\end{array}\right), \quad \bar{\gamma}=\left(\begin{array}{l}
1 \\
1
\end{array}\right)
$$

Since $H^{1}\left(\mathcal{W}, \mathbb{Z}_{2}\right)=\mathbb{Z}_{2}$, there are two inequivalent spinor structures on $\mathcal{W}$. The trivial one is called the periodic, or Ramond, spin structure in the physics literature. The nontrivial one is known as the anti-periodic, or Neveu-Schwarz, spin structure. Both spin structures play a role in string theory, but for our purposes it will be sufficient to consider the NeveuSchwarz spin structure. The corresponding semi-spinor bundles on $\mathcal{W}$ will be denoted by the same letters $S^{+}, S^{-}$. The parity-reversed (i.e. odd) semi-spinor bundles will be denoted by $\Pi S^{+}, \Pi S^{-}$. More generally, $\Pi$ will denote the parity-reversal functor. The vector

space morphisms $\gamma$ and $\bar{\gamma}$ give rise to a pair of bundle morphisms $S^{-} \rightarrow T \mathcal{W} \otimes S^{+}$and $S^{+} \rightarrow T \mathcal{W} \otimes S^{-}$which we denote by the same letters. 
Let $M$ be a $C^{\infty}$ manifold endowed with a Riemannian metric $G$ and a real 2-form $B$. At this stage we do not require $B$ to be closed. The indices of the tangent bundle $T M$ will be denoted by $j, k, l, \ldots$ in the upper position. The indices of the cotangent bundle $T^{*} M$ will be denoted by the same letters in the lower position. Summation over repeating indices is always implied.

Let $X$ be a $C^{\infty}$ map from $\mathcal{W}$ to $M$. Let $\psi$ and $\bar{\psi}$ be $C^{\infty}$ sections of $X^{*} T M \oplus$ $\Pi S^{+}$and $X^{*} T M \oplus \Pi S^{-}$, respectively. $N=1$ supersymmetric $\sigma$-model with worldsheet $\mathcal{W}$ and target $(X, G, B)$ is a classical field theory on $\mathcal{W}$ defined by the action

$$
\begin{aligned}
& \frac{1}{4 \pi} \int_{\mathcal{W}} G_{j k}(X)\left(d X^{j} \wedge * d X^{k}\right)+\frac{1}{4 \pi} \int_{\mathcal{W}} B_{j k}(X)\left(d X^{j} \wedge d X^{k}\right)+ \\
& \frac{1}{4 \pi} \int_{\mathcal{W}}\left(G_{j k}(X) \psi^{j} i \bar{\gamma} \cdot \nabla \psi^{k}+G_{j k}(X) \bar{\psi}^{j} i \gamma \cdot \nabla \bar{\psi}^{k}+\frac{1}{2} R_{j k l m}(X) \psi^{j} \psi^{k} \bar{\psi}^{l} \bar{\psi}^{m}\right) d \Sigma .
\end{aligned}
$$

Here the covariant derivatives $\nabla \psi$ and $\nabla \bar{\psi}$ are sections of $X^{*} T M \otimes \Pi S^{ \pm} \otimes T^{*} \mathcal{W}$ defined as follows:

$$
\begin{aligned}
& \nabla \psi^{j}=D \psi^{j}+\left(\left\{\begin{array}{l}
j \\
k l
\end{array}\right\}+\frac{3}{2}\left(G^{-1}\right)^{j m}(d B)_{k l m}\right) d X^{k} \psi^{l}, \\
& \nabla \bar{\psi}^{j}=D \bar{\psi}^{j}+\left(\left\{\begin{array}{l}
j \\
k l
\end{array}\right\}-\frac{3}{2}\left(G^{-1}\right)^{j m}(d B)_{k l m}\right) d X^{k} \bar{\psi}^{l},
\end{aligned}
$$

where $\{j, k l\}$ are the Christoffel symbols constructed from $G$, and $D: S^{ \pm} \rightarrow S^{ \pm} \otimes T^{*} \mathcal{W}$ is the Levi-Civita covariant derivative constructed from $g . R_{j k l m}(X)$ is the curvature corresponding to the following connection 1-form on $M$

$$
\left(\left\{\begin{array}{l}
j \\
k l
\end{array}\right\}+\frac{3}{2}\left(G^{-1}\right)^{j m}(d B)_{k l m}\right) d x^{l} .
$$

In the last term in the action we used twice the natural $S O(1,1)$-invariant pairing $S^{+} \otimes$ $S^{-} \rightarrow \mathbb{C}$.

This complicated-looking action has an elegant reformulation in terms of superfields, i.e. maps from a super-Riemann surface to $M$ [8].

The extrema of the action (42) are given by the solutions of the Euler-Lagrange equations. In the case when all the fields are even, it is well known that the space of solutions of the Euler-Lagrange equations is a manifold with a natural symplectic structure. This statement remains true in the supersymmetric context (see e.g. [15]). In the present case the symplectic structure is given by

$$
\begin{aligned}
\frac{1}{2 \pi} \int_{\tau=\tau_{0}}\left(\delta X ^ { j } \wedge \delta \left(G_{j k}(X) \frac{\partial X^{k}}{\partial \tau}+\right.\right. & \left.B_{j k}(X) \frac{\partial X^{k}}{\partial \sigma}\right) \\
& \left.+i G_{j k}(X) \delta \psi^{j} \wedge \delta \psi^{k}+i G_{j k}(X) \delta \bar{\psi}^{j} \wedge \delta \bar{\psi}^{k}\right) d \sigma .
\end{aligned}
$$


Here we used the fact the Euler-Lagrange equations are second-order in time derivatives of $X$ and first-order in time derivatives of $\psi, \bar{\psi}$, and therefore a solution is completely determined by the values of $X, \partial X / \partial \tau, \psi$, and $\bar{\psi}$ on any circle $\tau=\tau_{0}$. One can check that the symplectic structure thus defined does not depend on $\tau_{0}$. The space of solutions endowed with this symplectic structure is called the phase space of the $\sigma$-model.

We are interested in the case when $M$ is a torus $T^{2 d}=\mathbb{R}^{2 d} / \Gamma, \quad \Gamma \cong \mathbb{Z}^{2 d}$, with a constant metric $G$ and a constant 2 -form $B$. We will fix an isomorphism between $\Gamma$ and $\mathbb{Z}^{2 d}$. Without loss of generality we may assume that the action of $\Gamma$ on $\mathbb{R}^{2 d}$ is

$$
x^{j} \mapsto x^{j}+2 \pi n^{j}, \quad n^{j} \in \mathbb{Z}, \quad j=1,2, \ldots, 2 d .
$$

In this special case the $\sigma$-model action becomes

$$
\begin{aligned}
\frac{1}{4 \pi} \int_{\mathcal{W}}\left(G _ { j k } \left(\frac{\partial X^{j}}{\partial \tau} \frac{\partial X^{k}}{\partial \tau}\right.\right. & \left.-\frac{\partial X^{j}}{\partial \sigma} \frac{\partial X^{k}}{\partial \sigma}\right)+2 B_{j k} \frac{\partial X^{j}}{\partial \tau} \frac{\partial X^{k}}{\partial \sigma} \\
& \left.+i G_{j k} \psi^{j}\left(\frac{\partial}{\partial \tau}+\frac{\partial}{\partial \sigma}\right) \psi^{k}+i G_{j k} \bar{\psi}^{j}\left(\frac{\partial}{\partial \tau}-\frac{\partial}{\partial \sigma}\right) \bar{\psi}^{k}\right) d \tau d \sigma .
\end{aligned}
$$

The Euler-Lagrange equations have a simple form:

$$
\left(\frac{\partial^{2}}{\partial \sigma^{2}}-\frac{\partial^{2}}{\partial \tau^{2}}\right) X^{j}=0, \quad\left(\frac{\partial}{\partial \sigma}+\frac{\partial}{\partial \tau}\right) \psi^{j}=0, \quad\left(\frac{\partial}{\partial \sigma}-\frac{\partial}{\partial \tau}\right) \bar{\psi}^{j}=0 .
$$

In what follows we will use the notation

$$
\partial_{-}=\frac{1}{2}\left(\frac{\partial}{\partial \sigma}-\frac{\partial}{\partial \tau}\right), \quad \partial_{+}=\frac{1}{2}\left(\frac{\partial}{\partial \sigma}+\frac{\partial}{\partial \tau}\right) .
$$

The Poisson brackets of the fields evaluated at equal times follow from (43):

$$
\begin{aligned}
\left\{X^{j}(\tau, \sigma), X^{k}\left(\tau, \sigma^{\prime}\right)\right\}_{P . B .} & =0 \\
\left\{X^{j}(\tau, \sigma), \frac{\partial X^{k}}{\partial \tau}\left(\tau, \sigma^{\prime}\right)\right\}_{P . B .} & =2 \pi\left(G^{-1}\right)^{j k} \delta\left(\sigma-\sigma^{\prime}\right), \\
\left\{\psi(\tau, \sigma), \bar{\psi}\left(\tau, \sigma^{\prime}\right)\right\}_{P . B .} & =0, \\
\left\{\psi(\tau, \sigma), \psi\left(\tau, \sigma^{\prime}\right)\right\}_{P . B .} & =-2 \pi i\left(G^{-1}\right)^{j k} \delta\left(\sigma-\sigma^{\prime}\right) \\
\left\{\bar{\psi}(\tau, \sigma), \bar{\psi}\left(\tau, \sigma^{\prime}\right)\right\}_{P . B .} & =-2 \pi i\left(G^{-1}\right)^{j k} \delta\left(\sigma-\sigma^{\prime}\right) .
\end{aligned}
$$

The Poisson brackets between even and odd fields vanish.

Note that the neither the Euler-Lagrange equations (45) nor the symplectic structure corresponding to (46) depend on $B$. This happens whenever $B$ is closed, because in this case the $B$-dependent terms in the action are locally total derivatives. We will see below that quantization of the $\sigma$-model introduces arbitrariness which is parametrized by a class 
in $H^{2}(M, \mathbb{R} / \mathbb{Z})$. The usual interpretation is that while the classical $\sigma$-model does not detect a closed B-field, the quantized $\sigma$-model detects the image of $B$ in $H^{2}(M, \mathbb{R} / \mathbb{Z})$.

The Euler-Lagrange equations (45) can be rewritten in the Hamiltonian form:

$$
\begin{aligned}
\frac{\partial X^{j}}{\partial \tau}(\tau, \sigma) & =\left\{X^{j}(\tau, \sigma), H(\tau)\right\}_{P . B .}, \\
\frac{\partial}{\partial \tau}\left(\frac{\partial X^{j}}{\partial \tau}\right)(\tau, \sigma) & =\left\{\left(\frac{\partial X^{j}}{\partial \tau}\right)(\tau, \sigma), H(\tau)\right\}_{P . B .}, \\
\frac{\partial \psi^{j}}{\partial \tau}(\tau, \sigma) & =\left\{\psi^{j}(\tau, \sigma), H(\tau)\right\}_{P . B .}, \\
\frac{\partial \bar{\psi}^{j}}{\partial \tau}(\tau, \sigma) & =\left\{\bar{\psi}^{j}(\tau, \sigma), H(\tau)\right\}_{P . B .} .
\end{aligned}
$$

The Hamiltonian $H$ is a function on the phase space given by

$$
H\left(\tau_{0}\right)=\frac{1}{4 \pi} \int_{\tau=\tau_{0}} G_{j k}\left(\frac{\partial X^{j}}{\partial \tau} \frac{\partial X^{k}}{\partial \tau}+\frac{\partial X^{j}}{\partial \sigma} \frac{\partial X^{k}}{\partial \sigma}-i \psi^{j} \frac{\partial \psi^{k}}{\partial \sigma}+i \bar{\psi}^{j} \frac{\partial \bar{\psi}^{k}}{\partial \sigma}\right) d \sigma .
$$

As a consequence of the equations of motion, we have $\frac{d H\left(\tau_{0}\right)}{d \tau_{0}}=0$.

Hamiltonian vector fields on the phase space are those vector fields which preserve the symplectic form. They obviously form a Lie (super-)algebra with respect to the Lie bracket. We will now exhibit a subalgebra in this super-algebra which is isomorphic to the direct sum of two copies of the $N=1$ super-Virasoro algebra.

Recall that given a function $W$ on the phase space, we can define a Hamiltonian vector field $v_{W}$ as follows:

$$
v_{W}(\cdot)=\{\cdot, W\}_{P . B}
$$

One has an identity

$$
\left[v_{W}, v_{U}\right]_{\text {Lie }}=v_{\{W, U\}_{P . B .}} .
$$

We will define a set of functions on the phase space which forms a super-Virasoro algebra with respect to the Poisson bracket; then the corresponding set of Hamiltonian vector fields forms a super-Virasoro algebra with respect to the Lie bracket.

The set of functions we want to define is a vector space generated over $\mathbb{C}$ by the following elements:

$$
\begin{array}{rlrl}
L_{n} & =\frac{1}{2 \pi} \int_{\tau=\tau_{0}} e^{-i n \sigma} G_{j k}\left(\partial_{-} X^{j} \partial_{-} X^{k}-\frac{i}{2} \psi \partial_{-} \psi\right) d \sigma, & & n \in \mathbb{Z}, \\
\bar{L}_{n} & =\frac{1}{2 \pi} \int_{\tau=\tau_{0}} e^{i n \sigma} G_{j k}\left(\partial_{+} X^{j} \partial_{+} X^{k}+\frac{i}{2} \bar{\psi} \partial_{+} \bar{\psi}\right) d \sigma, & & n \in \mathbb{Z}, \\
Q_{r}=\frac{-i}{4 \pi} \int_{\tau=\tau_{0}} e^{-i r \sigma} G_{j k} \psi^{j} \partial_{-} X^{k} d \sigma, & r \in \mathbb{Z}+\frac{1}{2}, \\
\bar{Q}_{r}=\frac{i}{4 \pi} \int_{\tau=\tau_{0}} e^{i r \sigma} G_{j k} \bar{\psi}^{j} \partial_{-} X^{k} d \sigma, & r \in \mathbb{Z}+\frac{1}{2} .
\end{array}
$$


Two remarks are in order concerning these expressions. First, all these functions on the phase space implicitly depend on $\tau_{0}$ as a parameter. Second, since we picked the anti-periodic spin structure on $\mathcal{W}$, the lift of $\psi$ to the universal cover of $\mathcal{W}$ is an anti-periodic function of $\sigma$. This is the reason the index $r$ runs over half-integers.

The Poisson brackets of the generators can be easily computed using (46), and the nonvanishing ones turn out to be

$$
\begin{array}{ll}
\left\{L_{m}, L_{n}\right\}_{P . B .}=-i(m-n) L_{m+n}, & \left\{\bar{L}_{m}, \bar{L}_{n}\right\}_{P . B .}=-i(m-n) \bar{L}_{m+n}, \\
\left\{L_{m}, Q_{r}\right\}_{P . B .}=-i\left(\frac{m}{2}-r\right) Q_{m+r}, & \left\{\bar{L}_{m}, \bar{Q}_{r}\right\}_{P . B .}=-i\left(\frac{m}{2}-r\right) \bar{Q}_{m+r}, \\
\left\{Q_{r}, Q_{s}\right\}_{P . B .}=-\frac{i}{2} L_{r+s}, & \left\{\bar{Q}_{r}, \bar{Q}_{s}\right\}_{P . B .}=-\frac{i}{2} \bar{L}_{r+s},
\end{array}
$$

Thus the space spanned by the generators is a Lie super-algebra isomorphic to the direct sum of two copies of the $N=1$ super-Virasoro algebra (with zero central charge).

Note that $L_{0}+\bar{L}_{0}=H$. Recalling that the $\tau$-dependence of any function $F$ on the phase space is determined by

$$
\frac{d F}{d \tau}=\{F, H\}_{P . B .},
$$

and using (48), one can show that all the generators have a very simple dependence on $\tau_{0}$ :

$$
\begin{aligned}
L_{n}\left(\tau_{0}\right) & =e^{-i n \tau_{0}} L_{n}(0), & \bar{L}_{n}\left(\tau_{0}\right) & =e^{-i n \tau_{0}} \bar{L}_{n}(0), \\
Q_{r}\left(\tau_{0}\right) & =e^{-i r \tau_{0}} Q_{r}(0), & \bar{Q}_{r}\left(\tau_{0}\right) & =e^{-i r \tau_{0}} \bar{Q}_{r}(0) .
\end{aligned}
$$

Thus the space spanned by the generators does not depend on $\tau_{0}$.

The presence of two copies of the $N=1$ super-Virasoro algebra acting on the phase space is a feature of the supersymmetric $\sigma$-model with an arbitrary target $(M, G, B)$. This fact is crucial for string theory applications of the $\sigma$-model, see [29] for details.

Now let us choose a constant complex structure $I$ on $M$ such that $G$ is a Hermitian metric. This makes $M$ a Kähler manifold. Let $\omega=G I$ be the corresponding Kähler form. It turns out that we can embed each of the two $N=1$ super-Virasoro algebras in a bigger $N=2$ super-Virasoro algebra. The additional generators are given by

$$
\begin{array}{rlrl}
Q_{r}^{ \pm} & =\frac{-i}{8 \pi} \int_{\tau=\tau_{0}} e^{-i(r+1 / 2) \sigma}\left(G_{j k} \mp i \omega_{j k}\right) \psi^{j} \partial_{-} X^{k} d \sigma, & r \in \mathbb{Z}+\frac{1}{2}, \\
\bar{Q}_{r}^{ \pm}=\frac{i}{8 \pi} \int_{\tau=\tau_{0}} e^{i(r+1 / 2) \sigma}\left(G_{j k} \mp i \omega_{j k}\right) \bar{\psi}^{j} \partial_{+} X^{k} d \sigma, & r \in \mathbb{Z}+\frac{1}{2}, \\
J_{n}=\frac{-i}{4 \pi} \int_{\tau=\tau_{0}} e^{-i n \sigma} \omega_{j k} \psi^{j} \psi^{k} d \sigma, & n \in \mathbb{Z}, \\
\bar{J}_{n}=\frac{-i}{4 \pi} \int_{\tau=\tau_{0}} e^{i n \sigma} \omega_{j k} \bar{\psi}^{j} \bar{\psi}^{k} d \sigma, & n \in \mathbb{Z} .
\end{array}
$$


Note that $Q_{r}=Q_{r}^{+}+Q_{r}^{-}$and $\bar{Q}_{r}=\bar{Q}_{r}^{+}+\bar{Q}_{r}^{-}$for all $r$. The Poisson brackets between $L_{n}, Q_{r}^{ \pm}$, and $J_{n}$ are given by

$$
\begin{aligned}
& \left\{L_{m}, Q_{r}^{ \pm}\right\}_{P . B .}=-i\left(\frac{m}{2}-r\right) Q_{r+m}^{ \pm}, \\
& \left\{L_{m}, J_{n}\right\}_{P . B .}=i n J_{n+m}, \\
& \left\{Q_{r}^{+}, Q_{s}^{+}\right\}_{P . B .}=\left\{Q_{r}^{-}, Q_{r}^{-}\right\}_{P . B .}=0, \\
& \left\{Q_{r}^{+}, Q_{s}^{-}\right\}_{P . B .}=-\frac{i}{4} L_{r+s}-\frac{i}{8}(r-s) J_{r+s}, \\
& \left\{J_{m}, Q_{r}^{ \pm}\right\}_{P . B .}=\mp i Q_{r+m}^{ \pm} .
\end{aligned}
$$

The Poisson brackets between the barred generators have the same form. The Poisson brackets between barred and unbarred generators are trivial, as usual.

Again, the emergence of the $N=2$ super-Virasoro is not limited to the particular situation we are considering: one can prove that the phase space of the supersymmetric $\sigma$-model is acted upon by the $N=2$ super-Virasoro if $(M, G)$ is an arbitrary Kähler manifold, and $B$ is closed [1]. The statement can be further generalized to B-fields which are not closed 13.

Let us now look more closely at the space of solutions of the Euler-Lagrange equations. Note that any map $X: \mathcal{W} \rightarrow M$ induces a homomorphism of the homology groups $H_{1}(\mathcal{W}) \rightarrow H_{1}(M)$. The group $H_{1}(\mathcal{W}) \cong \pi_{1}(\mathcal{W}) \cong \mathbb{Z}$ has a preferred generator, namely the loop winding the $\mathbb{S}^{1}$ in the direction of increasing $\sigma$. Since $H_{1}\left(T^{2 d}\right)=\Gamma$, we see that to any map $X: \mathcal{W} \rightarrow M$ we can assign an element $w(X)$ of $\Gamma$. The components of $w$ are the so-called winding numbers of the map $X$. Thus the phase space of the $\sigma$-model is a disconnected sum

$$
\mathcal{M}=\bigsqcup_{w \in \Gamma} \mathcal{M}_{w}
$$

We will see in a moment that $\mathcal{M}_{w}$ is connected for all $w$.

The Euler-Lagrange equations (45) are linear and can be solved by Fourier transform. The general solution in $\mathcal{M}_{w}$ is given by

$$
\begin{aligned}
X^{j} & =x^{j}+\sigma w^{j}+\tau\left(G^{-1}\right)^{j k} p_{k}+\frac{i}{\sqrt{2}} \sum_{s=-\infty}^{\infty} \frac{1}{s}\left(\alpha_{s}^{j} e^{i s(\sigma-\tau)}+\bar{\alpha}_{s}^{j} e^{-i s(\sigma+\tau)}\right), \\
\psi^{j} & =\sum_{r \in \mathbb{Z}+1 / 2} \psi_{r}^{j} e^{i r(\sigma-\tau)} \\
\bar{\psi}^{j} & =\sum_{r \in \mathbb{Z}+1 / 2} \bar{\psi}_{r}^{j} e^{-i r(\sigma+\tau)} .
\end{aligned}
$$

Here $\alpha_{s}^{j}, \bar{\alpha}_{s}^{j}$ are complex numbers satisfying $\left(\alpha_{s}^{j}\right)^{*}=\alpha_{-s}^{j},\left(\bar{\alpha}_{s}^{j}\right)^{*}=\bar{\alpha}_{-s}^{j} ; \quad \psi_{r}^{j}, \bar{\psi}_{r}^{j}$ are elements of $\Pi \mathbb{C} ; x^{j}, j=1, \ldots, 2 d$, take values in $\mathbb{R} /(2 \pi \mathbb{Z}) ;$ and $p_{j}, i=1, \ldots, 2 d$, 
take values in $\mathbb{R}$. The variables $\alpha_{s}^{j}, \bar{\alpha}_{s}^{j}, \psi_{r}^{j}, \bar{\psi}_{r}^{j}$ will be referred to as "the oscillators." The variables $\left(x^{j}, p_{j}\right), j=1, \ldots, 2 d$, together parametrize a copy of $T^{*} M \cong T^{2 d} \times \mathbb{R}^{2 d}$.

Thus for any $w \in \Gamma$ the supermanifold $\mathcal{M}_{w}$ is a product of the vector superspace spanned by $\alpha_{n}, \bar{\alpha}_{n}, n \in \mathbb{Z}, \psi_{r}, \bar{\psi}_{r}, r \in \mathbb{Z}+1 / 2$, and the cotangent bundle of $M$.

The Poisson brackets of the coordinates on $\mathcal{M}_{w}$ can be computed from (46) and (50 52). The non-vanishing ones are given by

$$
\begin{array}{cc}
\left\{\alpha_{n}^{j}, \alpha_{m}^{k}\right\}_{P . B .} & =-i n\left(G^{-1}\right)^{j k} \delta_{m+n}, \quad\left\{\bar{\alpha}_{n}^{j}, \bar{\alpha}_{m}^{k}\right\}_{P . B .}=-i n\left(G^{-1}\right)^{j k} \delta_{m+n}, \\
\left\{\psi_{r}^{j}, \psi_{s}^{k}\right\}_{P . B .}=-i\left(G^{-1}\right)^{j k} \delta_{r+s}, & \left\{\bar{\psi}_{r}^{j}, \bar{\psi}_{s}^{k}\right\}_{P . B .}=-i\left(G^{-1}\right)^{j k} \delta_{r+s .} . \\
\left\{x^{j}, p_{k}\right\}_{P . B .}=\delta_{k}^{j},
\end{array}
$$

Thus the symplectic supermanifold $\mathcal{M}_{w}$ decomposes into a product of a symplectic vector superspace spanned by the oscillators and $T^{*} M$ with the standard symplectic structure.

It is customary to continue analytically the time variable $\tau$ to the imaginary axis. If we set $\tau=i t$, then the combination $v=\sigma+\tau=\sigma+i t$ becomes a complex variable. Since we identify $\sigma \sim \sigma+2 \pi$, it is convenient to set $v=i \log z$ where $z \in \mathbb{C}^{*}$. After analytic continuation $\partial_{-}$and $\partial_{+}$become $\partial_{v}=-i z \partial_{z}$ and $\bar{\partial}_{v}=i \bar{z} \bar{\partial}_{z}$, respectively. The functions $X^{j}(v(z))$ are multi-valued functions of $z$ if $w \neq 0$. But their derivatives with respect to $z$ and $\bar{z}$ are single-valued, and moreover are holomorphic and anti-holomorphic, respectively:

$$
\begin{aligned}
& \frac{\partial X^{j}}{\partial z}=-\frac{i}{2 z}\left(\left(G^{-1}\right)^{j k} p_{k}-w^{j}\right)-\frac{i}{\sqrt{2}} \sum_{s=-\infty}^{\infty} \frac{\alpha_{s}^{j}}{z^{s+1}}, \\
& \frac{\partial X^{j}}{\partial \bar{z}}=-\frac{i}{2 \bar{z}}\left(\left(G^{-1}\right)^{j k} p_{k}+w^{j}\right)-\frac{i}{\sqrt{2}} \sum_{s=-\infty}^{\infty} \frac{\bar{\alpha}_{s}^{j}}{\bar{z}^{s+1}} .
\end{aligned}
$$

Note that after rescaling $X^{j} \rightarrow(i \sqrt{2}) X^{j}$ these expressions become formally the same as $(13,14)$, except that in $(13,14)$ the coordinates on the phase space $w^{k}, p_{k}, \alpha_{s}^{k}, \bar{\alpha}_{s}^{k}$ are replaced with the operators $W^{k}, M_{k}-B_{k l} W^{l}, \alpha_{s}^{k}, \bar{\alpha}_{s}^{k}$, respectively. This replacement is the quantization map discussed in more detail below.

Similarly, after analytic continuation to imaginary $\tau$, the sections $\psi^{j}$ and $\bar{\psi}^{j}$ become holomorphic and anti-holomorphic, respectively. One additional subtlety arises due to the fact that $\psi$ and $\bar{\psi}$ are sections of semi-spinor bundles. Thus the coordinate change $v \mapsto$ $z=e^{-i v}$ must be accompanied by $\psi^{j} \mapsto z^{-1 / 2} \psi^{j}$, and $\bar{\psi}^{j} \mapsto \bar{z}^{-1 / 2} \bar{\psi}^{j}$. This accounts for the shift $r \mapsto r+\frac{1}{2}$ between (51,52) and (15, 16).

Let us now turn to the quantization of the $\sigma$-model. This discussion provides a motivation for the constructions of Section 4 . 
Since the classical phase space is a disconnected sum of identical pieces labeled by $w \in \Gamma$, the quantum-mechanical Hilbert space will be a tensor sum of identical Hilbert spaces labeled by $w \in \Gamma$. Thus we only need to understand how to quantize the supermanifold $\mathcal{M}_{w}$. In turn, $\mathcal{M}_{w}$ decomposes as a product of $T^{*} M$ with the standard symplectic structure, and a vector superspace spanned by the oscillators.

The vector superspace spanned by the oscillators can be quantized using the well-known Fock-Bargmann prescription. The resulting Hilbert superspace is the so-called Fock space, i.e. the completion with respect to a suitable norm of the space of polynomials of even variables $a_{-s}^{i}, \bar{a}_{-s}^{i}, s=1,2, \ldots$, and odd variables $\theta_{-r}, \bar{\theta}_{-r}, r=1 / 2,3 / 2, \ldots$ We will denote this space of polynomials $\mathcal{H}_{\text {Fock }}$.

The quantization of $T^{*} M$ is also standard and yields the Hilbert space which is the completion of the space $C^{\infty}(M)$ of smooth functions on $M=\mathbb{R}^{2 d} / \Gamma$ with respect to an $L^{2}$ norm. Using Fourier transform, this Hilbert space can be identified with the completion of the group algebra of $\Gamma^{*}$ with respect to an $\ell^{2}$ norm.

Thus the quantization procedure sketched above leads to the Hilbert space which is a suitable completion of an infinite-dimensional superspace

$$
\oplus_{w \in \Gamma} \mathbb{C}\left[\Gamma^{*}\right] \otimes \mathcal{H}_{\text {Fock }}
$$

This can be written in a more symmetric form:

$$
\mathbb{C}\left[\Gamma \oplus \Gamma^{*}\right] \otimes \mathcal{H}_{\text {Fock }}
$$

For our purposes, only the superspace structure, and not the Hilbert space structure, is important. Thus we need not perform the completion procedure, and can take the above superspace as the state space of the $N=2$ superconformal vertex algebra corresponding to the supersymmetric $\sigma$-model. We will call this vector superspace the state space of the quantized $\sigma$-model.

Finding a suitable state space is but a part of the quantization problem. Quantizing a classical field theory usually requires finding a sufficiently large subset of functions on the phase space closed under the Poisson brackets, and a map from this subset to the set of linear operators on the state space, such that the Poisson brackets are mapped to $-i$ times the graded commutator. The choice of the subset of functions on the phase space is dictated by physical considerations. For example, for string theory applications it is imperative to have an $N=1$ super-Virasoro algebra acting on the state space. Thus the distinguished subset must include the generators of the $N=1$ super-Virasoro algebra (47) and their linear combinations. We will also require that the subset include the generators of the $N=2$ super-Virasoro (49). Usually one also requires that the distinguished subset 
include the fields in terms of which the classical action is written. In our case these are $X^{j}(\sigma, \tau), \psi^{j}(\sigma, \tau), \bar{\psi}^{j}(\sigma, \tau)$. One also wants the operator corresponding to the Hamiltonian $H=L_{0}+\bar{L}_{0}$ to have nonnegative spectrum.

To quantize the fields $X^{j}, \psi^{j}$, and $\bar{\psi}^{j}$ it is sufficient to quantize the oscillators and $\left(x^{j}, p_{j}\right)$ (the coordinates on $T^{*} M$ ). The Fock-Bargmann quantization map sends oscillators with negative subscripts to multiplication operators on the space of polynomials:

$$
\begin{array}{rlrl}
\alpha_{s}^{j} \mapsto a_{s}^{j}, & \bar{\alpha}_{s}^{j} \mapsto \bar{a}_{s}^{j}, & s=-1,-2, \ldots, \\
\psi_{r}^{j} \mapsto \theta_{r}^{j}, & \bar{\psi}_{r}^{j} \mapsto \bar{\theta}_{r}^{j}, & r & =-\frac{1}{2},-\frac{3}{2}, \ldots
\end{array}
$$

The oscillators with positive subscripts are mapped to differentiation operators on the space of polynomials:

$$
\begin{aligned}
\alpha_{s}^{j} \mapsto s\left(G^{-1}\right)^{j k} \frac{\partial}{\partial a_{-s}^{k}}, & \bar{\alpha}_{s}^{j} \mapsto s\left(G^{-1}\right)^{j k} \frac{\partial}{\partial \bar{a}_{-s}^{k}}, & s=1,2, \ldots, \\
\psi_{r}^{j} \mapsto\left(G^{-1}\right)^{j k} \frac{\partial}{\partial \theta_{-r}^{k}}, & \bar{\psi}_{r}^{j} \mapsto\left(G^{-1}\right)^{j k} \frac{\partial}{\partial \bar{\theta}_{-r}^{k}}, & r=\frac{1}{2}, \frac{3}{2}, \ldots .
\end{aligned}
$$

It is easy to see that the (graded) commutators between these operators are equal to $i$ times the Poisson brackets of their classical counterparts, as required.

The quantization of $\left(x^{j}, p_{j}\right)$ proceeds as follows. The function $x^{j}$ is a multi-valued function on the phase space and cannot be quantized. But any smooth function $f\left(x^{1}, \ldots, x^{2 d}\right)$ which is periodic, i.e. invariant with respect to shifts $x^{j} \rightarrow x^{j}+2 \pi n^{j}, n^{j} \in \mathbb{Z}$, is a univalued function on the phase space. The standard quantization of $T^{*} M$ maps such a function to a multiplication operator on $C^{\infty}(M)$ :

$$
f\left(x^{1}, \ldots, x^{2 d}\right) \mapsto f\left(x^{1}, \ldots, x^{2 d}\right) .
$$

Actually, the vector space we are dealing with is not just $C^{\infty}(M)$, but a $\Gamma$-graded vector space

$$
\mathfrak{F}=\oplus_{w \in \Gamma} C^{\infty}(M)
$$

and therefore we should quantize a pair $(f, w)$ rather than $f$. This leads to an important subtlety. If $w=0$, we can assign to $(f, w)$ a multiplication operator which acts on each of the $\Gamma$-homogeneous components of $\mathfrak{F}$ in an identical manner. On the other hand, if $w \neq 0$, it does not seem right to assign to it multiplication by $f$, since such a quantization procedure would map different classical functions to the same quantum-mechanical operator. A natural guess for the operator corresponding to $(f, w)$ is multiplication by $f$ followed by an operator $T_{w}$, where $T_{w}$ shifts the $\Gamma$-grading by $w$. This guess will be justified below. 
Under the standard quantization of $T^{*} M$, the function $p_{j}$ is mapped to a differentiation operator on $\mathfrak{F}$ :

$$
p_{j} \mapsto \hat{p}_{j}=-i \frac{\partial}{\partial x^{j}}
$$

If $\hat{f}_{w}$ is the quantum operator corresponding to the function $(f, w) \in \mathfrak{F}$, we have the commutation relation

$$
\left[\hat{f}_{w}, \hat{p}_{j}\right]=i \widehat{\left(\frac{\partial f}{\partial x^{j}}\right)_{w}} .
$$

This should be compared with the classical relation

$$
\left\{f(x), p_{j}\right\}_{P . B .}=\frac{\partial f(x)}{\partial x^{j}} .
$$

The Fourier transform which identifies the completion of $\mathfrak{F}$ with the completion of $\mathbb{C}\left[\Gamma \oplus \Gamma^{*}\right]$ sends $\hat{p}_{j}$ to the following operator $M_{j}$ on $\mathbb{C}\left[\Gamma \oplus \Gamma^{*}\right]$ :

$$
M_{j}:(w, m) \mapsto m_{j}(w, m), \quad \forall(w, m) \in \Gamma \oplus \Gamma^{*}
$$

Putting all this together, we obtain the quantization map for $\partial X^{j}, \bar{\partial} X^{j}, \quad \psi^{j}$, and $\bar{\psi}^{j}$. It is easy to check that this yields the expressions (13-16) of Section $\square$ with $B=0$ (after we rescale $X^{j}$ by a factor $i \sqrt{2}$ ).

Now we can also motivate the state-operator correspondence postulated in Section 4 . The main idea that the quantization map should send local classical observables to local quantum fields belonging to the image of $Y$. For example, $\partial X^{j}, \bar{\partial} X^{j}, \psi^{j}, \bar{\psi}^{j}$ and their derivatives are local classical observables, so the corresponding quantum fields must lie in the image of $Y$. These considerations explain the mapping of the states $\alpha_{-s}^{j}|v a c\rangle, \quad \bar{\alpha}_{-s}^{j}|v a c\rangle, \quad \psi_{-r}^{j}|v a c\rangle$, and $\bar{\psi}_{-r}^{j}|v a c\rangle$. Together with the axioms of vertex algebra, this uniquely fixes the mapping of other states in the subspace $w=m=0$. Other natural local classical observables are suitable exponentials of $X^{j}(z, \bar{z})$. (The classical field $X^{j}(z, \bar{z})$ itself is multi-valued and therefore should not be quantized.) Requiring that they map to local quantum fields fixes the form of $Y$ for all $(w, m) \in \Gamma \oplus \Gamma^{*}$. An interested reader is referred to 29] for details.

Another important ingredient is the quantization of the $N=2$ super-Virasoro algebra. Naively, one would like to define the quantum generators by the same formulas (47,49), but with the classical fields replaced by the quantum fields. This idea runs into an immediate problem since the products of quantum fields at the same point are not well-defined. The normal ordering prescription resolves this problem and leads to well-defined operators. One can easily check that this definition of the generators of the $N=2$ super-Virasoro is equivalent to the one given in Section $⿴$. The operators thus defined form an infinite-dimensional Lie super-algebra which is a central extension of the classical $N=2$ super-Virasoro (47,49). One can also check that the spectrum of $H=L_{0}+\bar{L}_{0}$ is nonnegative. 
It remains to explain how to include the effect of the B-field. As remarked above, a closed B-field does not affect the classical $\sigma$-model. However, the above quantization procedure admits a modification which depends on a class in $H^{2}(M, \mathbb{R} / \mathbb{Z})$. We wish to interpret this class as the cohomology class of the B-field.

The modification affects the quantization of $T^{*} M$ and consists in replacing the space of smooth functions on $\mathbb{R}^{2 d} / \Gamma$ with the space of smooth functions on $\mathbb{R}^{2 d}$ satisfying the following quasi-periodicity condition:

$$
f\left(x^{1}+2 \pi n^{1}, \ldots, x^{2 d}+2 \pi n^{2 d}\right)=e^{-2 \pi i B_{j k} n^{j} w^{k}} f\left(x^{1}, \ldots, x^{2 d}\right),
$$

where $B_{j k}$ is a real skew-symmetric matrix which we can interpret as an element of $H^{2}(M, \mathbb{R})$ in a natural manner. We will denote the space of such functions $C_{w}^{\infty}(M, B)$. It is clear that $C_{w}^{\infty}(M, B)$ depends only on the image of $B$ in $H^{2}(M, \mathbb{R} / \mathbb{Z})$. Thus the modification consists of replacing $\mathfrak{F}$ with the space

$$
\mathfrak{F}(B)=\oplus_{w \in \Gamma} C_{w}^{\infty}(M, B) .
$$

Fourier transform identifies a completion of $C_{w}^{\infty}(M, B)$ with a completion of $\mathbb{C}\left[\Gamma^{*}\right]$, as before, so the Hilbert space of the quantum theory is unaffected by $B$. But the map of the classical functions on the phase space to operators is affected.

First, the product of two quasi-periodic functions $f \in C_{w}^{\infty}(M, B)$ and $f^{\prime} \in C_{w^{\prime}}^{\infty}(M, B)$ belongs to the space $C_{w+w^{\prime}}^{\infty}(M, B)$. Hence the multiplication operators do not preserve the $\Gamma$-grading on $\mathfrak{F}(B)$. Rather, multiplication by $f \in C_{w}^{\infty}(M, B)$ shifts the grading by $w$. If we want the limit $B \rightarrow 0$ to be smooth, we have to postulate that even for $B=0$ multiplication by $f \in C_{w}^{\infty}(M, B)$ shifts the grading by $w$. This provides a justification for the guess made above. Second, while the function $p_{j}$ is still mapped according to (53), the Fourier transform of $\hat{p}_{j}$ is different from (54). Namely, it is easy to see that the Fourier transform of the differentiation operator on $C_{w}^{\infty}(M, B)$ is given by $M_{j}-B_{j k} w^{k}$. Putting these facts together, one obtains the quantization map for all classical fields in agreement with 13 -16).

\section{B The relation between vertex algebras and chiral algebras}

In this appendix we describe some properties of vertex algebras in the sense of Definition 3.3. Let $(V,|v a c\rangle, T, \bar{T}, Y)$ be a vertex algebra. We prove that the subspace of $V$ spanned by vectors which are mapped by $Y$ to meromorphic fields has a natural structure of a chiral algebra. Furthermore, anti-meromorphic fields form another chiral algebra, and these two chiral algebras supercommute with each other. We also describe an analogue of the Borcherds 
(or associativity) formula for vertex algebras. Finally, we show that any chiral algebra is a vertex algebra.

We start with the following useful lemma.

Lemma B.1 Let $N, M$ be integers and let $h_{j}, j=1, \ldots, K$ be distinct real numbers belonging to $[0,1)$. Suppose the following relation holds

$$
\sum_{j=1}^{K} i_{z, w} \frac{1}{(z-w)^{N+h_{j}}} i_{\bar{z}, \bar{w}} \frac{1}{(\bar{z}-\bar{w})^{M+h_{j}}} C_{j}(z, \bar{z}, w, \bar{w})=0,
$$

where $C_{j}(z, \bar{z}, w, \bar{w}) \in Q F_{2}(V)$. Then $C_{j}(z, \bar{z}, w, \bar{w}) \equiv 0$ for all $j$.

It is sufficient to prove the statement for $M=N=0$. Let $v \in V$ be an arbitrary vector. We are going to prove that the value of $C_{j}$ on $v$ vanishes for all $j$. To this end let us evaluate both sides of (55) on $v$ and set $w=z x$ and $\bar{w}=\bar{z} \bar{x}$. Since $C_{j} \in Q F_{2}(V)$, the expression $C_{j}(z, \bar{z}, z x, \bar{z} \bar{x})(v)$ can be written as

$$
\sum_{\alpha, \beta} f_{\alpha \beta}(x, \bar{x}) z^{-\alpha} \bar{z}^{-\beta}
$$

where each $f_{\alpha \beta}$ is a finite sum of fractional powers of $x, \bar{x}$ with coefficients in $V$. Hence the value of the left-hand side of Eq. (55) on $v$ is a sum

$$
\sum_{\alpha, \beta} z^{-\alpha} \bar{z}^{-\beta} \sum_{(\gamma, \delta) \in J_{\alpha \beta}} x^{-\gamma} \bar{x}^{-\delta} T_{\alpha \beta \gamma \delta}
$$

where $J_{\alpha \beta} \subset \mathbb{R}^{2}$ is a finite set for each $(\alpha, \beta)$. Each $T_{\alpha \beta \gamma \delta}$ has the form

$$
\sum_{j=1}^{K} i_{x} \frac{1}{(1-x)^{h_{j}}} i_{\bar{x}} \frac{1}{(1-\bar{x})^{h_{j}}} f_{j}(x, \bar{x}),
$$

where all $f_{j}$ are polynomials in $x, \bar{x}$ with coefficients in $V$, and $h_{j} \in[0,1)$ are distinct real numbers. The symbol $i_{x}$ (resp. $i_{\bar{x}}$ ) means "expand in a Taylor series around $x=0$ " (resp. $\quad \bar{x}=0$ ). To prove the lemma it sufficient to show that if the expression Eq. (57) is zero, then $f_{j} \equiv 0$ for all $j$. To prove this, we rewrite $f_{j}$ as a polynomial in $1-x$ and $1-\bar{x}$. Then Eq. (57) takes the form

$$
\sum_{l=1}^{L} i_{x} \frac{1}{(1-x)^{t_{l}}} i_{\bar{x}} \frac{1}{(1-\bar{x})^{s_{l}}} a_{l}
$$

where $\left(t_{l}, s_{l}\right)$ are distinct pairs of real numbers, and each $a_{l} \in V$ is a coefficient of some $f_{j}$. Let us denote this expression by $T$. We will show by induction in $L$ that if $T$ is equal to 0 then $a_{l}=0$ for all $l$. This will imply that $f_{j} \equiv 0$ for all $j$. The base of 
induction is evident. Suppose $a_{1} \neq 0$. Multiply $T$ by $i_{x}(1-x)^{t_{1}} i_{x}(1-\bar{x})^{s_{1}}$ and apply to the resulting expression an operator

$$
A(1-x) \partial_{x}+B(1-\bar{x}) \partial_{\bar{x}}
$$

where $A, B$ are arbitrary real numbers. We obtain a sum with $L-1$ terms:

$$
\sum_{l=2}^{L} i_{x} \frac{1}{(1-x)^{t_{l}}} i_{\bar{x}} \frac{1}{(1-\bar{x})^{s_{l}}}\left(A\left(t_{l}-t_{1}\right)+B\left(s_{l}-s_{1}\right)\right) a_{l}
$$

which is equal to 0 whenever $T=0$. Since $A$ and $B$ are arbitrary, by the induction hypotesis we get $a_{l}=0$ for $l=2, \ldots, L$. Consequently, $a_{1}$ is equal to 0 as well. This proves the lemma.

Theorem B.2 (Uniqueness theorem) Let $\mathcal{V}$ be a subspace in $Q F_{1}(V)$ which satisfies the following conditions:

1. any field $A(z, \bar{z}) \in \mathcal{V}$ is mutually local with all fields $Y(a), a \in V$;

2. all fields are creative, i.e. $A(z, \bar{z})|0\rangle \in V[[z, \bar{z}]]$.

Then the map

$$
\begin{aligned}
& s: \mathcal{V} \rightarrow V[[z, \bar{z}]], \\
& A(z, \bar{z}) \mapsto A(z, \bar{z})|0\rangle
\end{aligned}
$$

is injective.

Suppose $A(z, \bar{z})|0\rangle=0$. Take a vector $a \in V$ and consider $Y(a)$. From locality we know that

$$
Y(a)(z, \bar{z}) A(w, \bar{w})=\sum_{j=1}^{M} i_{z, w} \frac{1}{(z-w)^{h_{j}+N}} i_{\bar{z}, \bar{w}} \frac{1}{(\bar{z}-\bar{w})^{h_{j}+N}} C_{j}(z, \bar{z}, w, \bar{w}) .
$$

Hence we have

$$
\sum_{j=1}^{M} i_{z, w} \frac{1}{(z-w)^{h_{j}+N}} i_{\bar{z}, \bar{w}} \frac{1}{(\bar{z}-\bar{w})^{h_{j}+N}} C_{j}(z, \bar{z}, w, \bar{w})|0\rangle=0 .
$$

Using the arguments of Lemma B.1, we get $C_{j}(z, \bar{z}, w, \bar{w})|0\rangle=0$ for all $j$. Now from locality we obtain

$$
A(w, \bar{w}) Y(a)(z, \bar{z})|0\rangle=0 .
$$

This implies that $A(w, \bar{w}) a=0$ for any $a \in V$. Hence $A(w, \bar{w})=0$, and the theorem is proved. 
Corollary B.3 For any $a \in V$ the following identities hold:

$$
Y(T a)=\partial Y(a), \quad Y(\bar{T} a)=\bar{\partial} Y(a)
$$

Both fields $Y(T a)$ and $\partial Y(a)$ are mutually local with all fields $Y(b)$. Moreover we have

$$
Y(T a)|0\rangle=\partial Y(a)|0\rangle=T e^{T z+\bar{T} \bar{z}} a .
$$

Hence by the uniqueness theorem

$$
Y(T a)=\partial Y(a)
$$

The other identity is proved similarly.

We call a vector $a \in V$ meromorphic (resp. anti-meromorphic) if $Y(a)$ is meromorphic (resp. anti-meromorphic). To show that meromorphic and anti-meromorphic vectors form two supercommuting chiral algebras, it is sufficient to prove the following proposition.

Proposition B.4 Let $V$ be a vertex algebra. Then

1. the subspace of meromorphic vectors is closed with respect to $Y$ and $T$, i.e. $T(a)$ and $a_{(n)} b$ are meromorphic when $a \in V$ and $b \in V$ are meromorphic,

2. the OPE of two meromorphic fields $a(z)$ and $b(w)$ can be written in the form

$$
\begin{aligned}
a(z) b(w) & =i_{z, w} \frac{1}{(z-w)^{N}} C(z, w), \\
(-1)^{p(a) p(b)} b(w) a(z) & =i_{w, z} \frac{1}{(z-w)^{N}} C(z, w), \quad C(z, w) \in Q F_{2}(V),
\end{aligned}
$$

where $N$ is an integer,

3. If $a \in V$ is meromorphic and $b \in V$ is anti-meromorphic, then their OPE has the form

$$
a(z) b(\bar{w})=C(z, \bar{w}), \quad(-1)^{p(a) p(b)} b(\bar{w}) a(z)=C(z, \bar{w}), \quad C(z, \bar{w}) \in Q F_{2}(V) .
$$

Let us prove statement (1) of the proposition. From Corollary B.3 we infer that $a$ is meromorphic if and only if $\bar{T} a=0$. Since $T$ and $\bar{T}$ commute, this immediately implies that $T a$ is meromorphic when $a$ is meromorphic. Further, consider $Y(a) b$, where both $a$ and $b$ are meromorphic. We have

$$
\bar{T} Y(a) b=Y(a)(\bar{T} b)=0 .
$$

Hence $\bar{T}\left(a_{(n)} b\right)=0$, and all $a_{(n)} b$ are meromorphic as well.

Statements (2) and (3) of the proposition are special cases of a more general statement which we are going to prove. 
Proposition B.5 Let $a, b \in V$. If $a$ is meromorphic, then the OPE of $a(z)$ and $b(w, \bar{w})$ can be written in the form

$$
\begin{aligned}
a(z) b(w, \bar{w}) & =i_{z, w} \frac{1}{(z-w)^{N}} D(z, w, \bar{w}), \\
(-1)^{p(a) p(b)} b(w, \bar{w}) a(z) & =i_{w, z} \frac{1}{(z-w)^{N}} D(z, w, \bar{w}),
\end{aligned}
$$

where $D(z, w, \bar{w}) \in Q F_{2}(V)$, and $N$ is an integer.

This means that if a certain variable does not appear on the left-hand-side of the OPE, it does not appear on the right-hand-side either.

The general form of the OPE of $a(z)$ and $b(w, \bar{w})$ is

$$
a(z) b(w, \bar{w})=\sum_{i=1}^{M} i_{z, w} \frac{1}{(z-w)^{N+h_{i}}} i_{\bar{z}, \bar{w}} \frac{1}{(\bar{z}-\bar{w})^{N+h_{i}}} C_{i}(z, \bar{z}, w, \bar{w}),
$$

where $N \in \mathbb{Z}, \quad h_{i}, i=1, \ldots, M$, are distinct real numbers which belong to $[0,1)$, and $C_{i} \in Q F_{2}(V)$.

Let us act on both sides with an operator $(\bar{z}-\bar{w}) \frac{\partial}{\partial \bar{z}}$. We get

$$
0=\sum_{i=1}^{M} i_{z, w} \frac{1}{(z-w)^{N+h_{i}}} i_{\bar{z}, \bar{w}} \frac{1}{(\bar{z}-\bar{w})^{N+h_{i}}}\left(-\left(N+h_{i}\right)+(\bar{z}-\bar{w}) \frac{\partial}{\partial \bar{z}}\right) C_{i} .
$$

By Lemma B.1 we may conclude that for all $i$ we have

$$
\left(-\left(N+h_{i}\right)+(\bar{z}-\bar{w}) \frac{\partial}{\partial \bar{z}}\right) C_{i}=0 .
$$

Now let us show that $C_{i} \equiv 0$ if $h_{i} \neq 0$. Assume the converse. Then there is a vector $v \in V$ such that

$$
C_{i}(z, \bar{z}, w, \bar{w})(v)=\sum_{\alpha, \beta, \gamma, \delta} c_{(\alpha \beta \gamma \delta)} z^{-\alpha} \bar{z}^{-\beta} w^{-\gamma} \bar{w}^{-\delta} \neq 0
$$

Eq. (59) implies

$$
\left(N+h_{i}+\beta\right) c_{(\alpha \beta \gamma \delta)}=(\beta-1) c_{(\alpha, \beta-1, \gamma, \delta+1)}
$$

Since $C_{i} \in Q F_{2}(V)$, we can choose $\alpha, \beta, \gamma, \delta$ so that $c_{(\alpha, \beta, \gamma, \delta)} \neq 0$ and $c_{(\alpha, \beta-1, \gamma, \delta+1)}=0$. From Eq. (60) we find that $\beta=-\left(N+h_{i}\right)$. Furthermore, (60) implies that

$$
c_{(\alpha, \beta+k, \gamma, \delta-k)}=\left(\begin{array}{c}
\beta+k-1 \\
k
\end{array}\right) c_{(\alpha, \beta, \gamma, \delta)}=\left(\begin{array}{c}
-\left(N+h_{i}\right)+k-1 \\
k
\end{array}\right) c_{(\alpha, \beta, \gamma, \delta)}
$$

for all $k \in \mathbb{N}$. If $h_{i} \notin \mathbb{Z}$ then the vector $c_{(\alpha, \beta+k, \gamma, \delta-k)} \in V$ is nonzero for all $k \in \mathbb{N}$. But this contradicts the condition $C_{i} \in Q F_{2}(V)$. 
Since $h_{i} \in[0,1)$ for all $i$, and $h_{i} \neq h_{j}$ for $i \neq j$, we conclude that $C_{i}=0$ for all $i$ except maybe one, and for the latter value of $i$ we have $h_{i}=0$. In addition, for $c_{(\alpha, \beta+k, \gamma, \delta-k)}$ to be zero for $k>>0$, as required by the condition $C_{i} \in Q F_{2}(V)$, the integer $N$ must be nonnegative. Thus the OPE of $a(z)$ and $b(w, \bar{w})$ has the form

$$
a(z) b(w, \bar{w})=i_{z, w} \frac{1}{(z-w)^{N}} i_{\bar{z}, \bar{w}} \frac{1}{(\bar{z}-\bar{w})^{N}} C(z, \bar{z}, w, \bar{w})
$$

where $C(z, \bar{z}, w, \bar{w}) \in Q F_{2}(V)$ and $N \geq 0$.

Applying Eq. (59) to $C(z, \bar{z}, w, \bar{w})$ and differentiating it with respect to $\bar{z}$, we infer that

$$
C(z, \bar{z}, w, \bar{w})=\frac{1}{N !}(\bar{z}-\bar{w})^{N} \partial_{\bar{z}}^{N} C(z, \bar{z}, w, \bar{w}) \quad \text { and } \quad \partial_{\bar{z}}^{N+1} C(z, \bar{z}, w, \bar{w})=0 .
$$

For this reason the element $\frac{1}{N !} \partial_{\bar{z}}^{N} C(z, \bar{z}, w, \bar{w}) \in Q F_{2}(V)$ does not depend on $\bar{z}$. Let us denote it by $D(z, w, \bar{w})$. Then the OPE of $a(z)$ and $b(w, \bar{w})$ takes the form

$$
\begin{aligned}
a(z) b(w, \bar{w}) & =i_{z, w} \frac{1}{(z-w)^{N}} D(z, w, \bar{w}), \\
(-1)^{p(a) p(b)} b(w, \bar{w}) a(z) & =i_{w, z} \frac{1}{(z-w)^{N}} D(z, w, \bar{w}) .
\end{aligned}
$$

This completes the proof of Proposition B.4. As a corollary, we have:

Corollary B.6 Meromorphic and anti-meromorphic vectors form two supercommuting chiral algebras.

In the theory of chiral algebras an important role is played by the so-called Borcherds formula which expresses the OPE of any two fields $a(z)$ and $b(z)$ in the image of $Y$ through their normal ordered product and the Borcherds products $a_{(n)} b$. We will prove an analogue of the Borcherds formula for vertex algebras.

Note that any field $D(z, w, \bar{w}) \in Q F_{2}(V)$ meromorphic in the first variable can be expanded in a Taylor series in $(z-w)$ to an arbitrarily high order. This means that for any integer $K>0$ there exists a field $D_{K}(z, w, \bar{w}) \in Q F_{2}(V)$ such that

$$
D(z, w, \bar{w})=\left.\sum_{j=0}^{K-1} \frac{(z-w)^{j}}{j !} \frac{\partial^{j} D(z, w, \bar{w})}{\partial z^{j}}\right|_{z=w}+(z-w)^{K} D_{K}(z, w, \bar{w}) .
$$

To prove this, it is sufficient to show that for any $D(z, w, \bar{w}) \in Q F_{2}(V)$ we have

$$
D(z, w, \bar{w})-D(w, w, \bar{w})=(z-w) D_{1}(z, w, \bar{w})
$$

for some $D_{1}(z, w, \bar{w}) \in Q F_{2}(V)$. This fact is trivial. Note also that if $D \in Q F_{2}(V)$ contains fractional powers of $z$ (and therefore also depends on $\bar{z}$ ), the Taylor formula need not hold. 
Using the Taylor formula, the OPE (58) can be rewritten in the following form

$$
a(z) b(w, \bar{w})=\sum_{j=1}^{N} i_{z, w} \frac{1}{(z-w)^{j}} C_{j}(w, \bar{w})+D_{N}(z, w, \bar{w}),
$$

where $C_{j}(w, \bar{w}) \in Q F_{1}(V)$ for all $j, \quad D_{N}(z, w, \bar{w}) \in Q F_{2}(V)$. It is easy to see that $C_{j}$ and $D_{N}$ are uniquely defined by this formula.

Moreover it can be easily checked that $C_{n}(w, \bar{w})$ coincides with

$$
a(w)_{(n)} b(w, \bar{w}):=\operatorname{Res}_{z}\left((z-w)^{n-1}(a(z) b(w, \bar{w})-b(w, \bar{w}) a(z))\right)
$$

The analogue of the Borcherds formula provides explicit expressions for $C_{j}$ and $D_{N}$ in terms of $a$ and $b$ :

$$
C_{j}(w, \bar{w})=Y\left(a_{(j)} b\right)(w, \bar{w}), \quad j=1, \ldots, N, \quad D_{N}(z, w, \bar{w})=: a(z) b(w, \bar{w}): .
$$

Here the normal ordered product $: a(z) b(w, \bar{w}): \in Q F_{2}(V)$ is defined as follows. Let

$$
a(z)_{+}=\sum_{n \leq 0} a_{(n)} z^{-n}, \quad a(z)_{-}=\sum_{n>0} a_{(n)} z^{-n}
$$

Then the normal ordered product of $a(z)$ and $b(w, \bar{w})$ is defined by

$$
: a(z) b(w, \bar{w}):=a(z)_{+} b(w, \bar{w})+(-1)^{p(a) p(b)} b(w, \bar{w}) a(z)_{-} .
$$

Thus the OPE of a meromorphic field and an arbitrary field takes the form

$$
a(z) b(w, \bar{w})=\sum_{j=1}^{N} i_{z, w} \frac{1}{(z-w)^{j}} Y\left(a_{(j)} b\right)(w, \bar{w})+: a(z) b(w, \bar{w}): .
$$

Similarly, the OPE of an anti-meromorphic field and an arbitrary field is given by

$$
a(\bar{z}) b(w, \bar{w})=\sum_{j=1}^{N} i_{\bar{z}, \bar{w}} \frac{1}{(\bar{z}-\bar{w})^{j}} Y\left(a_{(j)} b\right)(w, \bar{w})+: a(\bar{z}) b(w, \bar{w}): .
$$

To prove the analogue of the Borcherds formula it is sufficient to show that $a(w)_{(n)} b(w, \bar{w})$ is mutually local with any $Y(c)$. Indeed, it can be easily checked that

$$
a(w)_{(n)} b(w, \bar{w})|0\rangle=Y\left(a_{(n)} b\right)(w, \bar{w})|0\rangle,
$$

and hence by the uniqueness theorem we obtain

$$
a(w)_{(n)} b(w, \bar{w})=Y\left(a_{(n)} b\right)(w, \bar{w}) .
$$


Lemma B.7 If $a \in V$ is meromorphic, then $a(z)_{(n)} b(z, \bar{z}), n \geq 1$ is mutually local with any $Y(c)$.

We have to prove that

$$
a(w)_{(n)} b(w, \bar{w})=\operatorname{Res}_{z}\left((z-w)^{n-1}(a(z) b(w, \bar{w})-b(w, \bar{w}) a(z))\right)
$$

is mutually local with any $Y(c)=c(z, \bar{z})$.

Let us consider

$$
A=\left(z_{1}-z_{2}\right)^{n-1}\left(a\left(z_{1}\right) b\left(z_{2}, \bar{z}_{2}\right) c\left(z_{3}, \bar{z}_{3}\right)-b\left(z_{2}, \bar{z}_{2}\right) a\left(z_{1}\right) c\left(z_{3}, \bar{z}_{3}\right)\right)
$$

and

$$
B=\left(z_{1}-z_{2}\right)^{n-1}\left(c\left(z_{3}, \bar{z}_{3}\right) a\left(z_{1}\right) b\left(z_{2}, \bar{z}_{2}\right)-c\left(z_{3}, \bar{z}_{3}\right) b\left(z_{2}, \bar{z}_{2}\right) a\left(z_{1}\right)\right) .
$$

We know that for some sufficiently large $r \in \mathbb{N}$ the following identities hold:

$$
\begin{aligned}
& \left(z_{1}-z_{2}\right)^{r} a\left(z_{1}\right) b\left(z_{2}, \bar{z}_{2}\right)=\left(z_{1}-z_{2}\right)^{r} b\left(z_{2}, \bar{z}_{2}\right) a\left(z_{1}\right), \\
& \left(z_{1}-z_{3}\right)^{r} a\left(z_{1}\right) c\left(z_{3}, \bar{z}_{3}\right)=\left(z_{1}-z_{3}\right)^{r} c\left(z_{3}, \bar{z}_{3}\right) a\left(z_{1}\right) .
\end{aligned}
$$

Now let us consider $\left(z_{2}-z_{3}\right)^{M}$. We have

$$
\left(z_{2}-z_{3}\right)^{M}=\sum_{s=0}^{M}\left(\begin{array}{c}
M \\
s
\end{array}\right)\left(z_{2}-z_{1}\right)^{M-r}\left(z_{1}-z_{3}\right)^{s} .
$$

Let us multiply $A$ with $\left(z_{2}-z_{3}\right)^{M}$, where $M \geq 2 r$. We get

$$
\sum_{s=0}^{M}\left(\begin{array}{c}
M \\
s
\end{array}\right)\left(z_{2}-z_{1}\right)^{M-r}\left(z_{1}-z_{3}\right)^{s} A .
$$

For $0 \leq s \leq r$ the s-th summand in this expression is 0 , because $\left(z_{1}-z_{2}\right)^{M-s}\left(z_{1}-z_{2}\right)^{n-1}=$ $\left(z_{1}-z_{2}\right)^{r^{\prime}}$ where $r^{\prime} \geq r$. Hence the expression is equal to

$$
\sum_{s=r+1}^{M}\left(\begin{array}{c}
M \\
s
\end{array}\right)\left(z_{2}-z_{1}\right)^{M-r}\left(z_{1}-z_{3}\right)^{s} A=
$$

$$
\begin{gathered}
\sum_{s=r+1}^{M}\left(\begin{array}{c}
M \\
s
\end{array}\right)\left(z_{2}-z_{1}\right)^{M-r}\left(z_{1}-z_{3}\right)^{s}\left(z_{1}-z_{2}\right)^{n-1}\left(a\left(z_{1}\right) b\left(z_{2}, \bar{z}_{2}\right) c\left(z_{3}, \bar{z}_{3}\right)-b\left(z_{2}, \bar{z}_{2}\right) a\left(z_{1}\right) c\left(z_{3}, \bar{z}_{3}\right)\right)= \\
\sum_{s=r+1}^{M}\left(\begin{array}{c}
M \\
s
\end{array}\right)\left(z_{2}-z_{1}\right)^{M-r}\left(z_{1}-z_{3}\right)^{s}\left(z_{1}-z_{2}\right)^{n-1}\left(a\left(z_{1}\right) b\left(z_{2}, \bar{z}_{2}\right) c\left(z_{3}, \bar{z}_{3}\right)-b\left(z_{2}, \bar{z}_{2}\right) c\left(z_{3}, \bar{z}_{3}\right) a\left(z_{1}\right)\right)= \\
\sum_{s=r+1}^{M}\left(\begin{array}{c}
M \\
s
\end{array}\right)\left(z_{2}-z_{1}\right)^{M-r}\left(z_{1}-z_{3}\right)^{s}\left(z_{1}-z_{2}\right)^{n-1}\left[a\left(z_{1}\right), b\left(z_{2}, \bar{z}_{2}\right) c\left(z_{3}, \bar{z}_{3}\right)\right] .
\end{gathered}
$$


In the same way we find that

$$
\left(z_{2}-z_{3}\right)^{M} B=\sum_{s=r+1}^{M}\left(\begin{array}{c}
M \\
s
\end{array}\right)\left(z_{2}-z_{1}\right)^{M-r}\left(z_{1}-z_{3}\right)^{s}\left[a\left(z_{1}\right), c\left(z_{3}, \bar{z}_{3}\right) b\left(z_{2}, \bar{z}_{2}\right)\right] .
$$

From our definition of a vertex algebra we know that

$$
\begin{aligned}
b\left(z_{2}, \bar{z}_{2}\right) c\left(z_{3}, \bar{z}_{3}\right) & =\sum_{j} i_{z_{2}, z_{3}} \frac{1}{\left(z_{2}-z_{3}\right)^{h_{j}+N}} i_{\bar{z}_{2}, \bar{z}_{3}} \frac{1}{\left(\bar{z}_{2}-\bar{z}_{3}\right)^{h_{j}+N}} E_{j}\left(z_{2}, \bar{z}_{2}, z_{3}, \bar{z}_{3}\right), \\
c\left(z_{3}, \bar{z}_{3}\right) b\left(z_{2}, \bar{z}_{2}\right) & =\sum_{j} i_{z_{3}, z_{2}} \frac{1}{\left(z_{2}-z_{3}\right)^{h_{j}+N}} i_{\bar{z}_{3}, \bar{z}_{2}} \frac{1}{\left(\bar{z}_{2}-\bar{z}_{3}\right)^{h_{j}+N}} E_{j}\left(z_{2}, \bar{z}_{2}, z_{3}, \bar{z}_{3}\right)
\end{aligned}
$$

for some $E_{j}$ from $Q F_{2}(V)$. Substituting these expressions into the formulas above we find that

$$
\begin{gathered}
\left(z_{2}-z_{3}\right)^{M}\left(a\left(z_{2}\right)_{(n)} b\left(z_{2}, \bar{z}_{2}\right)\right) c\left(z_{3}, \bar{z}_{3}\right)=\operatorname{Res}_{z_{1}}\left(\sum_{s=r+1}^{M}\left(\begin{array}{c}
M \\
s
\end{array}\right)\left(z_{2}-z_{1}\right)^{M-r}\left(z_{1}-z_{3}\right)^{s}\left(z_{1}-z_{2}\right)^{n-1}\right. \\
\left.\sum_{j} i_{z_{2}, z_{3}} \frac{1}{\left(z_{2}-z_{3}\right)^{h_{j}+N}} i_{\bar{z}_{2}, \bar{z}_{3}} \frac{1}{\left(\bar{z}_{2}-\bar{z}_{3}\right)^{h_{j}+N}}\left[a\left(z_{1}\right), E_{j}\left(z_{2}, \bar{z}_{2}, z_{3}, \overline{z_{3}}\right)\right]\right),
\end{gathered}
$$

and

$$
\begin{gathered}
\left(z_{2}-z_{3}\right)^{M} c\left(z_{3}, \bar{z}_{3}\right) a\left(z_{2}\right)_{(n)} b\left(z_{2}, \bar{z}_{2}\right)=\operatorname{Res}_{z_{1}}\left(\sum_{s=r+1}^{M}\left(\begin{array}{c}
M \\
s
\end{array}\right)\left(z_{2}-z_{1}\right)^{M-r}\left(z_{1}-z_{3}\right)^{s}\left(z_{1}-z_{2}\right)^{n-1}\right. \\
\sum_{j} i_{z_{3}, z_{2}} \frac{1}{\left(z_{2}-z_{3}\right)^{h_{j}+N}} i_{\bar{z}_{3}, \bar{z}_{2}} \frac{1}{\left(\bar{z}_{2}-\bar{z}_{3}\right)^{h_{j}+N}}\left[a\left(z_{1}\right), E_{j}\left(z_{2}, \bar{z}_{2}, z_{3}, \overline{z_{3}}\right)\right]
\end{gathered}
$$

To prove mutual locality of $a(z)_{(n)} b(z, \bar{z})$ with any $Y(c)$ one only needs to show that one can divide both sides of the above equations by $\left(z_{2}-z_{3}\right)^{M}$. In fact, it is sufficient to show this for $M=1$, and then use induction on $M$.

To show that one can divide both sides by $z_{2}-z_{3}$, we note that the kernel of multiplication by $z-w$ consists of expressions of the form

$$
\sum_{n \in \mathbb{Z}}\left(\frac{z}{w}\right)^{n} D(z, \bar{z}, w, \bar{w}),
$$

where $D(z, \bar{z}, w, \bar{w})$ is a formal fractional power series with coefficients in $\operatorname{End}(V)$ (but not necessarily an element of $\left.Q F_{2}(V)\right)$. If $D(z, \bar{z}, w, \bar{w})$ is not identically zero, then there exists $v \in V$ such that when this expression is applied to $v$, one gets a fractional power series with coefficients in $V$ containing arbitrarily large negative powers of $w$ and $z$. On the other hand, applying any element of $Q F_{1}(V)$ or $Q F_{2}(V)$ to any $v \in V$ one always obtains a fractional power series with powers bounded from below. This implies that one can divide both sides of the above equations by $z_{2}-z_{3}$. The Borcherds formulas are proven. 
Three remarks are in order here. First, it seems that there is no analogous way to rewrite the OPE of two fields when neither of them is meromorphic or anti-meromorphic. Consequently, the normal ordered product of two general fields is not a very useful concept.

Second, given two meromorphic fields, one can define two normal ordered products:

$$
\begin{aligned}
& : a(z) b(w):=a(z)_{+} b(w)+(-1)^{p(a) p(b)} b(w) a(z)_{-}, \\
& : b(w) a(z):=b(w)_{+} a(z)+(-1)^{p(a) p(b)} a(z) b(w)_{-} .
\end{aligned}
$$

Correspondingly, there are two different OPEs that one can write down. The first one is

$$
\begin{aligned}
a(z) b(w) & =\sum_{j=1}^{N} i_{z, w} \frac{1}{(z-w)^{j}} Y\left(a_{(j)} b\right)(w)+: a(z) b(w):, \\
(-1)^{p(a) p(b)} b(w) a(z) & =\sum_{j=1}^{N} i_{w, z} \frac{1}{(z-w)^{j}} Y\left(a_{(j)} b\right)(w)+: a(z) b(w):,
\end{aligned}
$$

and the second one is

$$
\begin{aligned}
b(w) a(z) & =\sum_{j=1}^{N} i_{w, z} \frac{1}{(w-z)^{j}} Y\left(b_{(j)} a\right)(z)+: b(w) a(z): \\
(-1)^{p(a) p(b)} a(z) b(w) & =\sum_{j=1}^{N} i_{z, w} \frac{1}{(w-z)^{j}} Y\left(b_{(j)} a\right)(z)+: b(w) a(z): .
\end{aligned}
$$

In general, the two normal ordered products are not related in any simple way.

Third, given a meromorphic and an anti-meromorphic field, one can also define two normal ordered products. However, in this case they always coincide up to a sign:

$$
: a(z) b(\bar{w}):=(-1)^{p(a) p(b)}: b(\bar{w}) a(z): .
$$

Indeed, the OPE formulas (62,63) read in this case

$$
\begin{aligned}
& a(z) b(\bar{w})=(-1)^{p(a) p(b)} b(\bar{w}) a(z)=: a(z) b(\bar{w}):, \\
& b(\bar{w}) a(z)=(-1)^{p(a) p(b)} a(z) b(\bar{w})=: b(\bar{w}) a(z): .
\end{aligned}
$$

This fact also follows directly from the definition of the normal ordered product and the fact that meromorphic and anti-meromorphic fields in the image of $Y$ supercommute.

Finally, let us show that any chiral algebra is a special case of a vertex algebra with $\bar{T}=0$ and the image of $Y$ consisting of meromorphic fields only. The only thing which needs to be checked is the OPE axiom. For a chiral algebra, the OPE of any two fields in the image of $Y$ has the form

$$
\begin{aligned}
a(z) b(w) & =\sum_{n=1}^{N} i_{z, w} \frac{1}{(z-w)^{n}} Y\left(a_{(n)} b\right)(w)+: a(z) b(w): \\
(-1)^{p(a) p(b)} b(w) a(z) & =\sum_{n=1}^{N} i_{w, z} \frac{1}{(z-w)^{n}} Y\left(a_{(n)} b\right)(w)+: a(z) b(w):
\end{aligned}
$$


Obviously, $a_{(n)} b(w)$ belongs to $Q F_{2}(V)$. It is also easy to check that : $a(z) b(w)$ : also belongs to $Q F_{2}(V)$. Hence, the above $\mathrm{OPE}$ can be rewritten as

$$
a(z) b(w)=i_{z, w} \frac{1}{(z-w)^{N}} C(z, w)
$$

where $C(z, w) \in Q F_{2}(V)$. Therefore the OPE axiom is satisfied.

\section{Projectively flat connections and the fundamental group}

In this appendix we establish a relation between projectively flat connections on complex vector bundles on a connected manifold and finite representations of a twisted group algebra of the fundamental group. This relation is a generalization of the well-known statement that flat connections on complex vector bundles are in one-to-one correspondence with representations of the fundamental group.

Let $M$ be a paracompact connected $C^{\infty}$-manifold. Let us fix a closed real 2-form $B$ on $M$. Consider a complex vector bundle $E$ on $M$ with a connection $\nabla$ such that its curvature $F_{\nabla} \in \Omega^{2} \otimes \operatorname{End}(E)$ is equal to

$$
F_{\nabla}=2 \pi i B \otimes i d_{E}
$$

Such a connection is called projectively flat, and it is flat if and only if $B=0$. When $B$ is non-zero, we can consider the condition (64) as a "twisted" variant of the flatness condition.

We will prove that the set of such connections is in one-to-one correspondence with finite representations of a twisted group algebra of $\pi_{1}(M)$ defined below.

Let us fix a point $x \in M$. Since $(E, \nabla)$ is projectively flat, for any contractible closed path $c$ starting at $x$ the holonomy operator $H_{c}: E_{x} \longrightarrow E_{x}$ is equal to $t_{c} \cdot i d$, where $t_{c}$ is a nonzero complex number. By the Reduction Theorem (see [22]) (E, $\nabla)$ can be reduced locally to a $\mathbb{C}^{*}$-bundle, and therefore by Stockes' theorem

$$
t_{c}=\exp \left(2 \pi i \int_{D} \phi^{*} B\right)
$$

where $\phi$ is a map from the two dimensional disk $D$ to $M$ satisfying $\phi(\partial D)=c$. Since $B$ is a real 2 -form, $(E, \nabla)$ in fact locally reduces to a $U(1)$-bundle.

The above formula for $t_{c}$ is independent of the choice of $\phi$ only if

$$
\exp \left(2 \pi i \int_{S^{2}} \phi^{*} B\right)=1
$$

for any map $\phi$ from the 2-dimensional sphere $S^{2}$ to $M$. Thus a vector bundle $(E, \nabla)$ with curvature $F_{\nabla}=2 \pi i B \otimes i d_{E}$ can exist only if the de Rham cohomology class of $B$ belongs to the kernel of the composition homomorphism

$$
H^{2}(M, \mathbb{R}) \rightarrow H^{2}(M, U(1)) \rightarrow \operatorname{Hom}\left(\pi_{2}(M), U(1)\right) .
$$


Let us consider the Hopf sequence

$$
\pi_{2}(M) \longrightarrow H_{2}(M, \mathbb{Z}) \longrightarrow H_{2}(K(G, 1), \mathbb{Z}) \longrightarrow 0,
$$

where $G:=\pi_{1}(M)$. This sequence induces an injective map

$$
0 \longrightarrow H^{2}(K(G, 1), U(1)) \longrightarrow H^{2}(M, U(1)) \text {. }
$$

Denote by $\mathcal{B}$ the image of $B$ in $H^{2}(M, U(1))$. We showed that if $\mathcal{B}$ does not belong to the image of the map (66) then the set of vector bundles $(E, \nabla)$ with curvature $F_{\nabla}=$ $2 \pi i B \otimes i d_{E}$ is empty.

Assume now that $\mathcal{B}$ is in the image of the map (66). Let us fix a point $x \in M$ and for each element $g \in G$ choose a closed path $c_{g}$ beginning at $x$ and representing $g$ such that the closed path $c_{g^{-1}}$ coincides with the inverse of $c_{g}$ for any $g$. Let $c_{(g, h)}$ be a loop which is the union of the loops $c_{h}, c_{g}$, and $c_{(g h)^{-1}}$ This loop is contractible. Define a function $\psi: G \times G \rightarrow U(1)$ by the rule

$$
\psi(g, h)=\exp \left(2 \pi i \int_{D} \phi^{*} B\right)
$$

where $\phi$ is a map from the two dimensional disc $D$ to $M$ satisfying $\phi(\partial D)=c_{(g, h)}$. It is easy to see that this function is a 2-cocycle on the group $G$. Moreover, if we choose the representatives $c_{g}$ differently, we obtain a cocycle which is cohomologous to $\psi$.

The holonomy operators along the loops $c_{g}, c_{h}$, and $c_{g h}$ satisfy the following relation

$$
H_{c_{g}} \cdot H_{c_{h}}=\psi(g, h) H_{c_{g h}}
$$

This identity has the following representation-theoretic meaning. With any 2-cocycle $\psi$ one can associate a twisted group algebra $\mathbb{C}_{\psi}[G]$, which is a vector space generated by the elements $g \in G$ with the following multiplication law:

$$
g \cdot h=\psi(g, h) g h
$$

(Note that if two 2-cocycles are cohomological to each other, then the corresponding twisted group algebras are isomorphic.) The holonomy operators $H_{c_{g}}$ define a representation of the twisted group algebra $\mathbb{C}_{\psi}[G]$ on the vector space $E_{x}$.

An equivalent definition of the algebra $\mathbb{C}_{\psi}[G]$ goes as follows. Let $L p_{x}$ be the loop space of $M$ with the well-known composition of loops (which is associative only up to a homotopy). Let us consider the corresponding non-associative "group" algebra $\mathbb{C}\left[L p_{x}\right]$. Then the algebra $\mathbb{C}_{\psi}[G]$ is a factor-algebra of $\mathbb{C}\left[L p_{x}\right]$ modulo all relations of the form

$$
c-\exp \left(2 \pi i \int_{D} \phi^{*} B\right) \cdot 1=0
$$


where $c$ is a contractible loop, and $\phi$ is a map from the disc $D$ to $M$ such that $\phi(\partial D)=c$. By $($ 65) this definition does not depend on the choice of $\phi$. For any loop $c \in L p_{x}$ we denote by $r(c)$ the element of the twisted group algebra which is the image of $c$ with respect to this factorization.

In this way to any vector bundle $(E, \nabla)$ satisfying the condition (64) we can associate a finite-dimensional representation of the twisted group algebra. We assert that this is a one-to-one correspondence. To show this, we describe how to construct $(E, \nabla)$ starting from a representation $R$ of the twisted group algebra.

Let $C_{M}$ be the sheaf of algebras of complex-valued $C^{\infty}$-functions on $M$. Let $\mathcal{A}$ be a sheaf of algebras on $M$ defined as $\mathbb{C}_{\psi}[G] \otimes_{\mathbb{C}} C_{M}$. If $R$ is a representation of the twisted group algebra, then the sheaf $\mathcal{R}=R \otimes_{\mathbb{C}} C_{M}$ has a natural left module structure over the sheaf of algebras $\mathcal{A}$. Below we construct a sheaf $\mathcal{P}$ of right $\mathcal{A}$-modules with a connection $\nabla_{\mathcal{P}}$ and set $E=\mathcal{P} \otimes_{\mathcal{A}} \mathcal{R}$. This sheaf is the sheaf of sections of a complex vector bundle on $M$, and $\nabla_{P}$ induces a natural connection $\nabla$ on it.

Let $\widetilde{M} \stackrel{\tau}{\longrightarrow} M$ be a universal covering. Denote by $\widetilde{B}$ the pull-back of the form $B$ to $\widetilde{M}$. It is easy to check that $B$ belongs to the image of the map (66) if and only if $\widetilde{B}$ is an exact form. Let us choose a 1 -form $\eta$ on $\widetilde{M}$ such that $d \eta=\widetilde{B}$.

Consider a sheaf of algebras $\widetilde{\mathcal{A}}=\mathbb{C}_{\psi}[G] \otimes_{\mathbb{C}} C_{\widetilde{M}}$ on $\widetilde{M}$. The tautological action of $G$ on $\widetilde{M}$ can be lifted to a left action on $\widetilde{\mathcal{A}}$ as follows. Let $c_{g}$ be a loop in $M$ based at a fixed point $x \in M$ and representing the element $g \in G$, and let $r\left(c_{g}\right)$ be the corresponding element of the twisted group algebra of $G$ (see above). Let $x_{0}$ be a lift of $x$ to $\widetilde{M}$. Let $\widetilde{c}_{g}$ be a path on $\widetilde{M}$ which covers $c_{g}$, begins at $g^{-1}\left(x_{0}\right)$ and ends at $x_{0}$. For any point $y \in \widetilde{M}$ let us choose some path $d_{y}$ from $y$ to $x_{0}$. Let $\widetilde{c}_{g, y}$ be a path from $g^{-1}(y)$ to $y$ which is a composition of $g^{-1}\left(d_{y}\right), \quad \widetilde{c}_{g}$, and $d_{y}^{-1}$. The left action of the group $G$ on the sheaf $\widetilde{\mathcal{A}}$ is defined by the rule:

$$
g(a \otimes f)(y)=\exp \left(-2 \pi i \int_{\widetilde{c}_{g, y}} \eta\right)\left(r\left(c_{g}\right) a \otimes f\left(g^{-1} y\right)\right)
$$

where $a \in \mathbb{C}_{\psi}[G]$ and $f$ is a $C^{\infty}$-function on $\widetilde{M}$.

This definition does not depend on the choice of $d_{y}$, because the form $\widetilde{B}$ is $G$ invariant. Nor does it depend on the choice of $c_{g}$, because for any other loop $c_{g}^{\prime}$ representing $g$ we have

$$
\exp \left(-2 \pi i \int_{\widetilde{c}_{g, y}^{\prime}} \eta\right) r\left(c_{g}^{\prime}\right)=\exp \left(-2 \pi i \int_{\widetilde{c}_{g, y}^{\prime}} \eta+2 \pi i \int_{D} \phi^{*} \widetilde{B}\right) r\left(c_{g}\right)=\exp \left(-2 \pi i \int_{\widetilde{c}_{g, y}} \eta\right) r\left(c_{g}\right),
$$

where $\phi$ is a map from $D$ to $\widetilde{M}$ such that $\phi(\partial D)$ is the composition of $\widetilde{c}_{g}$ and the inverse of $\widetilde{c}_{g}$. 
Furthermore, we can define a connection on $\widetilde{\mathcal{A}}$ by the formula

$$
\widetilde{\nabla}(a \otimes f)=a \otimes(d f+2 \pi i f \eta)
$$

This connection is $G$-invariant. Indeed, let us regard $\int_{\widetilde{c}_{g, y}} \eta$ as a function on $\widetilde{M}$ and denote it by $h(y)$. Then we have

$$
\begin{aligned}
g \widetilde{\nabla}(a \otimes f)(y) & =g(a \otimes(d f+2 \pi i f \eta))(y) \\
& =\exp (-2 \pi i h(y)) r\left(c_{g}\right) a \otimes\left(d f\left(g^{-1} y\right)+2 \pi i f\left(g^{-1} y\right) \eta\left(g^{-1} y\right)\right) .
\end{aligned}
$$

On the other hand, since $d h(y)=\eta(y)-\eta\left(g^{-1} y\right)$ we obtain

$$
\begin{aligned}
\widetilde{\nabla} g(a \otimes f)(y) & =\widetilde{\nabla}\left(r\left(c_{g}\right) a \otimes \exp (-2 \pi i h(y)) f\left(g^{-1} y\right)\right) \\
& =\exp (-2 \pi i h(y)) r\left(c_{g}\right) a \otimes\left(d f\left(g^{-1} y\right)-2 \pi i f\left(g^{-1} y\right) d h(y)+2 \pi i f\left(g^{-1} y\right) \eta(y)\right) \\
& =\exp (-2 \pi i h(y)) r\left(c_{g}\right) a \otimes\left(d f\left(g^{-1} y\right)+2 \pi i f\left(g^{-1} y\right) \eta\left(g^{-1} y\right)\right) .
\end{aligned}
$$

The definitions of the connection $\widetilde{\nabla}$ and the action of the group $G$ on $\widetilde{\mathcal{A}}$ depend on the choice of $\eta$. However, if we take another form $\eta^{\prime}=\eta+d f$ then the data $(\widetilde{\mathcal{A}}, \widetilde{\nabla})$ and $\left(\widetilde{\mathcal{A}}, \widetilde{\nabla}^{\prime}\right)$ are isomorphic under the multiplication by the function $\exp (-2 \pi i f)$. Moreover, this isomorphism is compatible with the action of the group $G$.

We define a sheaf $\mathcal{P}$ on $M$ as the sheaf of invariants $\tau_{*}(\widetilde{\mathcal{A}})^{G}$ with a connection $\nabla_{\mathcal{P}}$ induced by $\widetilde{\nabla}$.

The sheaf $\mathcal{P}$ has a right module structure over $\mathcal{A}$. It is locally free of rank 1 as an $\mathcal{A}$-module. It follows from the preceding discussion that the datum $\left(\mathcal{P}, \nabla_{\mathcal{P}}\right)$ is unique and depends only on the form $B$.

To any representation $R$ of the twisted group algebra of $G$ we attach a complex vector bundle $E=\mathcal{P} \otimes_{\mathcal{A}} \mathcal{R}$ with the connection $\nabla$ induced by $\nabla_{\mathcal{P}}$. It is easy to see that the representation of the twisted group algebra on the space $E_{x}$ corresponding to $\nabla$ is isomorphic to $R$. Thus pairs $(E, \nabla)$ satisfying (64) are in one-to-one correspondence with finite-dimensional representations of $\mathbb{C}_{\psi}[G]$, where the cocycle $\psi$ is defined by (67).

\section{Acknowledgements}

We are grateful to Maxim Kontsevich for valuable comments and to Markus Rosellen for pointing out a gap in the reasoning of Appendix B in the first version of the paper. We also wish to thank the Institute for Advanced Study, Princeton, NJ, for a very stimulating atmosphere. The first author was supported by DOE grant DE-FG02-90ER40542. The second author was supported in part by RFFI grant 99-01-01144 and a grant for support of leading scientific groups N 00-15-96085. The research described in this publication was made 
possible in part by Award No RM1-2089 of the U.S. Civilian Research and Development Foundation for the Independent States of the Former Soviet Union (CRDF).

\section{References}

[1] L. Alvarez-Gaume and D. Freedman, Geometrical structure and ultraviolet finiteness in the supersymmetric sigma-model, Comm. Math. Phys. 80, 443 (1981).

[2] D. Arinkin and A. Polishchuk, Fukaya category and Fourier transform, preprint math//9811023.

[3] V. I. Arnold, Sturm theorem and symplectic geometry, Funkts. Anal. i Prilozh. 19, no. 4, p. 1 (1985).

[4] P. Bouwknegt and V. Mathai, D-branes, B-fields and twisted K-theory, JHEP 0003, 007 (2000); preprint hep-th/0002023.

[5] A. Belavin, A. Polyakov, and A. Zamolodchikov, Infinite conformal symmetries in twodimensional quantum field theory, Nucl. Phys. B241, 333 (1984).

[6] R. Borcherds, Vertex algebras, Kac-Moody algebras, and the Monster, Proc. Natl. Acad. Sci. USA 83, 3068 (1986).

[7] A. Connes, M. Douglas, and A. Schwarz, Noncommutative geometry and Matrix theory: compactification on tori, JHEP 9802, 003 (1998); preprint hep-th/9711162.

[8] P. Deligne et al., Quantum fields and strings: a course for mathematicians. Vol. 1,2, Providence, USA: AMS (1999).

[9] P. Di Francesco, P. Mathieu, and D. Sénéchal, Conformal field theory, New York: Springer (1997).

[10] M. Douglas, D-branes on Calabi-Yau manifolds, preprint math//0009209.

[11] M. Douglas and C. Hull, D-branes and the noncommutative torus, JHEP 9802, 008 (1998); preprint hep-th/9711165.

[12] K. Fukaya, Morse Homotopy, $A_{\infty}$-category and Floer Homologies, MSRI preprint No. 020-94 (1994).

[13] S. J. Gates, C. Hull, and M. Roček, Twisted multiplets and new supersymmetric nonlinear sigma-models, Nucl. Phys. B248, 157 (1984). 
[14] V. Golyshev, V. Lunts, and D. Orlov, Mirror symmetry for abelian varieties, to appear in Journal of Algebraic Geometry, preprint math//9912003.

[15] M. Henneaux and C. Teitelboim, Quantization of gauge systems, Princeton, NJ: Princeton University Press, (1992).

[16] C. Hofman and W.K. Ma, Deformations of topological open strings, preprint hepth/0006120.

[17] R. Hoobler, Brauer groups of abelian schemes, Ann. Sci. Ec. Norm. Sup. 5, 45 (1972).

[18] K. Hori, A. Iqbal, and C. Vafa, D-branes and mirror symmetry, preprint hepth/0005247.

[19] V. Kac, Vertex algebras for beginners, University Lecture Series, v.10, Providence, USA: AMS (1997).

[20] A. Kapustin, D-branes in a topologically nontrivial B-field, Adv. Theor. Math. Phys. 4, 127 (2000); preprint hep-th/9909089.

[21] B. Keller, Introduction to $A_{\infty}$-algebras and modules, preprint math//9910179.

[22] S. Kobayashi and K. Nomizu, Foundations of differential geometry. Vol. 1,2, New York: John Wiley \& Sons (1996).

[23] M. Kontsevich, Homological algebra of mirror symmetry, Proceedings of ICM (Zurich, 1994), p. 120-139, Basel: Birkhäuser (1995).

[24] D. Lüst and S. Theisen, Lectures on string theory, Berlin-Heidelberg: Springer-Verlag (1989).

[25] K. Narain, M. Sarmadi, and E. Witten, A note on toroidal compactification of heterotic string theory, Nucl. Phys. B279, 369 (1987).

[26] H. Ooguri, Y. Oz, and Z. Yin, D-branes on Calabi-Yau spaces and their mirrors, Nucl. Phys. B477, 407 (1996); preprint hep-th/9606112.

[27] D. Orlov, Equivalences of derived categories and K3 surfaces, Journal of Math. Sciences 84, 1361 (1997); preprint math//9606006.

[28] D. Orlov, On equivalences of derived categories of coherent sheaves on abelian varieties, preprint MPI/97-49, math//9712017. 
[29] J. Polchinski, String theory. Vol. 1,2, Cambridge, UK: Cambridge University Press, (1998).

[30] A. Polishchuk, Symplectic biextensions and generalization of the Fourier-Mukai transforms, Math. Res. Let. 3, 813 (1996); preprint math//9511018.

[31] A. Polishchuk and E. Zaslow, Categorical mirror symmetry: the elliptic curve, Adv. Theor. Math. Phys. 2, 443 (1998); preprint math/9801119.

[32] C. Vafa, Extending mirror conjecture to Calabi-Yau with bundles, preprint hepth/9804131.

[33] E. Witten, Chern-Simons gauge theory as a string theory, preprint hep-th/9207094.

[34] T. Kugo and B. Zwiebach, Target space duality as a symmetry of string field theory, Progr. Theor. Phys. 87, 801 (1992); preprint hep-th/9201040. 